\title{
A Modular Approach to Arylazo-1,2,3-triazole Photoswitches
}

Jeremy R. Tuck, ${ }^{\dagger}$ Robert J. Tombari, ${ }^{\dagger}$ Noah Yardeny, David E. Olson*

Supplementary Material

(73 pages)

Contents

1. General Information

2. Detailed Synthetic Procedures and Experimental Data for all Compounds

3. UV-Vis Characterization and Figure S1

4. Constant Illumination NMR Experiments

5. Thermal Stability and Half-life measurements

6. Supplemental References

7. ${ }^{1} \mathrm{H}$ and ${ }^{13} \mathrm{C}$ NMR Spectra 


\section{General Information}

All reagents were obtained from commercial sources and reactions were performed using ovendried glassware $\left(120^{\circ} \mathrm{C}\right)$ under an inert $\mathrm{N}_{2}$ atmosphere unless otherwise noted. Air- and moisture-sensitive liquids and solutions were transferred via syringe or stainless-steel cannula. Reactions that required heating were heated via an oil bath. Organic solutions were concentrated under reduced pressure ( $~ 5$ Torr) by rotary evaporation. Solvents were purified by passage under 12 psi $\mathrm{N}_{2}$ through activated alumina columns. Chromatography was performed using Fisher Chemical $^{\mathrm{TM}}$ Silica Gel Sorbent (230-400 Mesh, Grade 60). Compounds purified by chromatography were typically applied to the adsorbent bed using the indicated solvent conditions with a minimum amount of added dichloromethane as needed for solubility. Thin layer chromatography (TLC) was performed on Merck silica gel 60 F254 plates (250 $\mu \mathrm{m})$. Visualization of the developed chromatogram was accomplished by fluorescence quenching or by staining with butanolic ninhydrin, aqueous potassium permanganate, or aqueous ceric ammonium molybdate (CAM).

Nuclear magnetic resonance (NMR) spectra were acquired on either a Bruker 400 operating at 400 and $100 \mathrm{MHz}$, a Varian 600 operating at 600 and $150 \mathrm{MHz}$, or a Bruker 600 operating at 600 and $150 \mathrm{MHz}$ for ${ }^{1} \mathrm{H}$ and ${ }^{13} \mathrm{C}$, respectively, and are referenced internally according to residual solvent signals. Data for ${ }^{1} \mathrm{H}$ NMR are recorded as follows: chemical shift ( $\left.\delta, p p m\right)$, multiplicity (s, singlet; d, doublet; $t$, triplet; $q$, quartet; quint, quintet; $m$, multiplet), coupling constant $(\mathrm{Hz})$, and integration. Data for ${ }^{13} \mathrm{C} N M R$ are reported in terms of chemical shift $(\delta, \mathrm{ppm})$. High-resolution mass spectra were obtained using a Thermo Fisher Scientific Q-Exactive HF Orbitrap.

\section{Detailed Synthesis Procedures and Experimental Data for all Compounds}

$$
\text { Boc. }_{N^{H}} \cdot \stackrel{\mathrm{N}^{\mathrm{N}}}{\text { Boc }}
$$

\section{di-tert-butyl hydrazine-1,2-dicarboxylate (1)}

Compound 1 was synthesized using a modified literature procedure. ${ }^{1}$ To a $-10^{\circ} \mathrm{C}$ solution of $\mathrm{Boc}_{2} \mathrm{O}(40 \mathrm{~g}, 183.28 \mathrm{mmol}, 2.05$ equiv) in $\mathrm{MeOH}(50 \mathrm{~mL})$ was added a solution of hydrazine monohydrate $(4.30 \mathrm{~mL}, 88.65 \mathrm{mmol}, 1.0$ equiv) in $\mathrm{MeOH}(50 \mathrm{~mL})$ over $30 \mathrm{~min}$. The resulting mixture was allowed to stir at ambient temperature for $5 \mathrm{~h}$ then concentrated under reduced pressure. The resulting white solid was triturated in boiling hexanes $(160 \mathrm{~mL})$, cooled in a $-20^{\circ} \mathrm{C}$ freezer, and filtered. The white solid was washed with ice-cold hexanes $(2 \times 80 \mathrm{~mL})$ and dried in vacuo to afford compound $1(17.752 \mathrm{~g}, 86 \%)$ as a white solid. Spectral data matches that reported in the literature.

${ }^{1} \mathrm{H}$ NMR $\left(400 \mathrm{MHz}, \mathrm{CDCl}_{3}\right) \delta=6.23-5.98(\mathrm{~m}, 2 \mathrm{H}), 1.47(\mathrm{~s}, 18 \mathrm{H}) \mathrm{ppm}$.

${ }^{13} \mathrm{C}$ NMR $\left(100 \mathrm{MHz}, \mathrm{CDCl}_{3}\right) \delta=155.9,81.5,28.3 \mathrm{ppm}$.

HRMS $\left(\mathrm{ES}^{+}\right): m / z[\mathrm{M}+\mathrm{Na}]^{+}$calcd for $\mathrm{C}_{10} \mathrm{H}_{20} \mathrm{~N}_{2} \mathrm{O}_{4} \mathrm{Na}^{+} 255.1321$, found 255.1317.

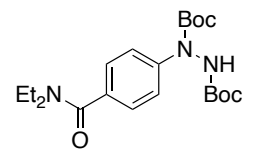

\section{di-tert-butyl 1-(4-(diethylcarbamoyl)phenyl)hydrazine-1,2-dicarboxylate (2a)}

A Schlenk tube was sequentially charged with compound 1 (1.0 g, $4.308 \mathrm{mmol}, 1.2$ equiv), $\mathrm{Cs}_{2} \mathrm{CO}_{3}$ (1.635 g, $5.026 \mathrm{mmol} 1.4$ equiv), 1,10-phenanthroline ( $0.129 \mathrm{~g}, 0.718 \mathrm{mmol}, 0.2$ equiv), copper(I) iodide ( $0.634 \mathrm{~g}, 3.590 \mathrm{mmol}, 1.0$ equiv), N,N-diethyl-4-iodobenzamide ${ }^{2}(1.088 \mathrm{~g}, 3.590$ $\mathrm{mmol}, 1.0 \mathrm{eq})$, and DMF $(4.49 \mathrm{~mL})$. The mixture was stirred and heated at $80^{\circ} \mathrm{C}$ for $36 \mathrm{~h}$, after 
which it was cooled to ambient temperature, diluted with EtOAc $(50 \mathrm{~mL})$, and filtered through celite. The reaction vessel and filter cake were washed with additional EtOAc. The filtrate was poured into water $(100 \mathrm{~mL})$ and the layers were separated. The aqueous layer was further extracted with EtOAc $(2 \times 50 \mathrm{~mL})$. The organic extracts were combined, washed with brine (100 $\mathrm{mL}$ ), dried over $\mathrm{Na}_{2} \mathrm{SO}_{4}$, filtered, and concentrated under reduced pressure. The residue was purified by chromatography on silica gel (50\% EtOAc in hexanes) to afford compound 2 a (1.370 g, 94\%) as a light yellow solid.

${ }^{1} \mathrm{H}$ NMR $\left(400 \mathrm{MHz} \mathrm{CDCl}_{3}\right) \delta=7.48-7.42(\mathrm{~m}, 2 \mathrm{H}), 7.33(\mathrm{~d}, \mathrm{~J}=13 \mathrm{~Hz}, 2 \mathrm{H}), 3.58-3.40(\mathrm{~m}, 2 \mathrm{H})$, 3.38-3.18 (m, 2H), 1.56-1.41 (m, 18H), 1.28-1.16 (m, 3H), 1.16-0.96 (m, 3H) ppm.

${ }^{13} \mathrm{C}$ NMR $\left(100 \mathrm{MHz} \mathrm{CDCl}_{3}\right) \delta=171.0,155.5,153.4,142.9,133.9,126.8,122.6,82.7,81.8,43.4$, 39.4, 28.3, 28.2, 14.3, $13.0 \mathrm{ppm}$.

HRMS $\left(\mathrm{ES}^{+}\right): \mathrm{m} / \mathrm{z}[\mathrm{M}+\mathrm{H}]^{+}$Calcd for $\mathrm{C}_{21} \mathrm{H}_{34} \mathrm{~N}_{3} \mathrm{O}_{5}{ }^{+}$408.2498; Found 408.2496.

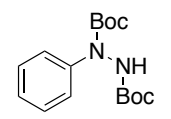

di-tert-butyl 1-phenylhydrazine-1,2-dicarboxylate (2b)

A Schlenk tube was sequentially charged with compound 1 (1.0 g, $4.308 \mathrm{mmol}, 1.2$ equiv), $\mathrm{Cs}_{2} \mathrm{CO}_{3}$ (1.635 g, $5.026 \mathrm{mmol} 1.4$ equiv), 1,10-phenanthroline ( $0.129 \mathrm{~g}, 0.718 \mathrm{mmol}, 0.2$ equiv), copper(I) iodide $(0.634 \mathrm{~g}, 3.590 \mathrm{mmol}, 1.0$ equiv), iodobenzene $(0.732 \mathrm{~g}, 3.590 \mathrm{mmol}, 1.0 \mathrm{eq})$, and DMF (4.49 mL). The mixture was stirred and heated at $80{ }^{\circ} \mathrm{C}$ for $24 \mathrm{~h}$, after which it was cooled to ambient temperature, diluted with EtOAc $(50 \mathrm{~mL})$, and filtered through celite. The reaction vessel and filter cake were washed with additional EtOAc. The filtrate was poured into water $(100 \mathrm{~mL})$ and the layers were separated. The aqueous layer was further extracted with EtOAc $(2 \times 50 \mathrm{~mL})$. The organic extracts were combined, washed with brine $(100 \mathrm{~mL})$, dried over $\mathrm{Na}_{2} \mathrm{SO}_{4}$, filtered, and concentrated under reduced pressure. The residue was purified by chromatography on silica gel (10\% EtOAc in hexanes) to afford compound $\mathbf{2 b}(1.083 \mathrm{~g}, 98 \%)$ as a white solid.

${ }^{1} \mathrm{H}$ NMR $\left(400 \mathrm{MHz}, \mathrm{CDCl}_{3}\right) \delta 7.44-7.35(\mathrm{~m}, 2 \mathrm{H}), 7.1(\mathrm{t}, \mathrm{J}=7.6 \mathrm{~Hz}, 2 \mathrm{H}), 7.14(\mathrm{t}, J=7.4 \mathrm{~Hz}, 1 \mathrm{H})$, 1.54-1.44 (m, 18H) ppm.

${ }^{13} \mathrm{C}$ NMR $\left(100 \mathrm{MHz}, \mathrm{CDCl}_{3}\right) \delta=155.6,153.7,142.3,128.5,125.7,123.8,82.4,81.7,28.3,28.3$ ppm.

HRMS $\left(\mathrm{ES}^{+}\right): \mathrm{m} / \mathrm{z}[\mathrm{M}+\mathrm{Na}]^{+}$Calcd for $\mathrm{C}_{16} \mathrm{H}_{24} \mathrm{~N}_{2} \mathrm{O}_{4} \mathrm{Na}^{+} 331.1634$; Found 331.1631.

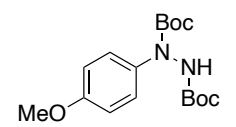

di-tert-butyl 1-(4-methoxyphenyl)hydrazine-1,2-dicarboxylate (2c)

A Schlenk tube was sequentially charged with compound 1 ( $4.5 \mathrm{~g}, 19.39 \mathrm{mmol}, 1.2$ equiv), $\mathrm{Cs}_{2} \mathrm{CO}_{3}$ (7.356 g, $22.62 \mathrm{mmol} 1.4$ equiv), 1,10-phenanthroline (0.582 g, $3.23 \mathrm{mmol}, 0.2$ equiv), copper(I) iodide (3.077 g, $16.15 \mathrm{mmol}, 1.0$ equiv), p-iodoanisole $(3.781 \mathrm{~g}, 16.15 \mathrm{mmol}, 1.0 \mathrm{eq})$, and DMF $(20.2 \mathrm{~mL})$. The mixture was stirred and heated at $80^{\circ} \mathrm{C}$ for $36 \mathrm{~h}$, after which it was cooled to ambient temperature, diluted with EtOAc $(250 \mathrm{~mL})$, and filtered through celite. The reaction vessel and filter cake were washed with additional EtOAc. The filtrate was poured into water (500 $\mathrm{mL})$ and the layers were separated. The aqueous layer was further extracted with EtOAc $(2 \times 250$ $\mathrm{mL})$. The organic extracts were combined, washed with brine $(500 \mathrm{~mL})$, dried over $\mathrm{Na}_{2} \mathrm{SO}_{4}$, 
filtered, and concentrated under reduced pressure. The residue was purified by chromatography on silica gel (DCM to $2 \%$ EtOAc in DCM) to afford compound $2 \mathrm{c}(5.455 \mathrm{~g},>99 \%)$ as a white solid. ${ }^{1} \mathrm{H}$ NMR $\left(400 \mathrm{MHz}, \mathrm{CDCl}_{3}\right) \delta=7.36-7.25(\mathrm{~m}, 2 \mathrm{H}), 6.83(\mathrm{~d}, \mathrm{~J}=9 \mathrm{~Hz}, 2 \mathrm{H}), 3.78(\mathrm{~s}, 3 \mathrm{H}), 1.51-1.44$ $(\mathrm{m}, 18 \mathrm{H}) \mathrm{ppm}$.

${ }^{13} \mathrm{C}$ NMR $\left(100 \mathrm{MHz}, \mathrm{CDCl}_{3}\right) \delta=157.7,155.6,154.2,135.5,126.2,113.8,82.1,81.5,55.6,28.3$, $28.3 \mathrm{ppm}$.

HRMS $\left(\mathrm{ES}^{+}\right): \mathrm{m} / \mathrm{z}[\mathrm{M}+\mathrm{Na}]^{+}$Calcd for $\mathrm{C}_{17} \mathrm{H}_{26} \mathrm{~N}_{2} \mathrm{O}_{5} \mathrm{Na}^{+}$361.1739; Found 361.1735.

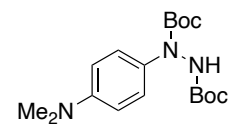

di-tert-butyl 1-(4-(dimethylamino)phenyl)hydrazine-1,2-dicarboxylate (2d)

A Schlenk tube was sequentially charged with compound 1 (1.410 g, $6.07 \mathrm{mmol}, 1.0$ equiv), $\mathrm{Cs}_{2} \mathrm{CO}_{3}$ (2.37 g, $7.28 \mathrm{mmol}, 1.2$ equiv), 1,10-phenanthroline ( $0.219 \mathrm{~g}, 1.21 \mathrm{mmol}, 0.2$ equiv), copper(I) iodide (1.156 g, $6.07 \mathrm{mmol}, 1.0$ equiv), 4-iodo- $\mathrm{N}, \mathrm{N}$-dimethylaniline $(1.5 \mathrm{~g}, 6.07 \mathrm{mmol}$, $1.0 \mathrm{eq})$, and DMF $(7.6 \mathrm{~mL})$. The mixture was stirred and heated at $80^{\circ} \mathrm{C}$ for $40 \mathrm{~h}$, after which it was cooled to ambient temperature, diluted with EtOAc $(300 \mathrm{~mL})$, and filtered through celite. The reaction vessel and filter cake were washed with additional EtOAc. The filtrate was poured into water $(500 \mathrm{~mL})$ and the layers were separated. The aqueous layer was further extracted with EtOAc $(2 \times 250 \mathrm{~mL})$. The organic extracts were combined, washed with brine $(500 \mathrm{~mL})$, dried over $\mathrm{Na}_{2} \mathrm{SO}_{4}$, filtered, and concentrated under reduced pressure. The residue was purified by chromatography on silica gel (5\% EtOAc in hexanes to $20 \%$ EtOAc in hexanes) to afford compound $2 \mathbf{d}(1.251 \mathrm{~g}, 59 \%)$ as an off-white solid.

${ }^{1} \mathrm{H}$ NMR $\left(400 \mathrm{MHz}, \mathrm{CDCl}_{3}\right) \delta=7.27-7.18(\mathrm{~m}, 2 \mathrm{H}), 6.66(\mathrm{~d}, \mathrm{~J}=9 \mathrm{~Hz}, 2 \mathrm{H}), 2.93(\mathrm{~s}, 6 \mathrm{H}), 1.52-1.42$ (m, 18H) ppm.

${ }^{13} \mathrm{C}$ NMR $\left(100 \mathrm{MHz}, \mathrm{CDCl}_{3}\right) \delta=155.6,154.5,149.2,132.0,126.2,112.5,81.7,81.3,40.9,28.4$, $28.3 \mathrm{ppm}$.

HRMS (ES $): m / z[M+H]^{+}$Calcd for $\mathrm{C}_{18} \mathrm{H}_{30} \mathrm{~N}_{3} \mathrm{O}_{4}{ }^{+}$352.2236: Found 352.2241.

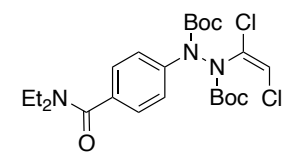

di-tert-butyl dicarboxylate (3a)

A flask was charged with sodium hydride (60\% dispersion in mineral oil) $(0.093 \mathrm{~g}, 2.32 \mathrm{mmol}, 2.1$ equiv), washed with hexanes, and dried under a stream of $\mathrm{N}_{2}$. A solution of $2 \mathrm{a}(0.450 \mathrm{~g}, 1.105$ mmol, 1.0 equiv) in DMF (4.4 mL) was added in one portion and the mixture was stirred at ambient temperature for $1 \mathrm{~h}$. Trichloroethylene $(0.105 \mathrm{~mL}, 1.160 \mathrm{mmol}, 1.05$ equiv) was added dropwise over $1 \mathrm{~h}$ and the solution was allowed to stir for an additional $1 \mathrm{~h}$. The solution was poured into water $(250 \mathrm{~mL})$ and extracted with EtOAc $(3 \times 100 \mathrm{~mL})$. The organic extracts were combined, washed with brine $(100 \mathrm{~mL})$, dried over $\mathrm{Na}_{2} \mathrm{SO}_{4}$, filtered, and concentrated under reduced pressure. The residue was purified by chromatography on silica gel (20\% EtOAc in hexanes) to afford compound $3 \mathrm{a}(0.539 \mathrm{~g}, 98 \%)$ as a white semi-solid.

${ }^{1} \mathrm{H}$ NMR $\left(400 \mathrm{MHz}, \mathrm{CDCl}_{3}\right)$ (rotamers observed) $\delta=7.39-7.31(\mathrm{~m}, 2 \mathrm{H}), 7.26-7.22(\mathrm{~m}, 2 \mathrm{H}), 6.05-$ $5.97(\mathrm{~m}, 1 \mathrm{H}), 3.46-3.28(\mathrm{~m}, 2 \mathrm{H}), 3.25-3.04(\mathrm{~m}, 2 \mathrm{H}), 1.44-1.38(\mathrm{~m}, 18 \mathrm{H}), 1.17-1.04(\mathrm{~m}, 3 \mathrm{H})$, 1.04-0.91 (m, 3H) ppm. 
${ }^{13} \mathrm{C}$ NMR $\left(100 \mathrm{MHz}, \mathrm{CDCl}_{3}\right)$ (rotamers observed) $\delta=170.5,170.4,152.2,152.0,150.3,149.5$, $140.5,135.3,129.5,129.0,127.0,126.6,126.5,125.1,124.6,115.4,114.6,83.7,83.0,82.7,43.1$, 39.1, 27.9, 27.9, 27.8, 27.8, 14.0, 14.0, $12.7 \mathrm{ppm}$.

HRMS $\left(\mathrm{ES}^{+}\right): \mathrm{m} / \mathrm{z}[\mathrm{M}+\mathrm{H}]^{+}$Calcd for $\mathrm{C}_{23} \mathrm{H}_{34} \mathrm{Cl}_{2} \mathrm{~N}_{3} \mathrm{O}_{5}{ }^{+}$502.1876; Found 502.1876.

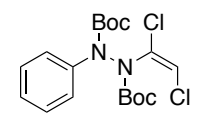

di-tert-butyl (E)-1-(1,2-dichlorovinyl)-2-phenylhydrazine-1,2-dicarboxylate (3b)

A flask was charged with sodium hydride (60\% dispersion in mineral oil) $(0.221 \mathrm{~g}, 5.538 \mathrm{mmol}$, 2.1 equiv), washed with hexanes, and dried under a stream of $\mathrm{N}_{2}$. A solution of $\mathbf{2 b}(0.813 \mathrm{~g}, 2.637$ mmol, 1.0 equiv) in DMF $(10.5 \mathrm{~mL})$ was added in one portion and stirred at ambient temperature for $1 \mathrm{~h}$. Trichloroethylene $(0.249 \mathrm{~mL}, 2.769 \mathrm{mmol}, 1.05$ equiv) was added dropwise over $1 \mathrm{~h}$ and allowed to stir for an additional $30 \mathrm{~min}$. The solution was poured into water $(150 \mathrm{~mL})$ and extracted with EtOAc $(3 \times 60 \mathrm{~mL})$. The organic extracts were combined, washed with brine $(150 \mathrm{~mL})$, dried over $\mathrm{Na}_{2} \mathrm{SO}_{4}$, filtered, and concentrated under reduced pressure. The residue was purified by chromatography on silica gel (6\% EtOAc in hexanes to $9 \%$ EtOAc in hexanes) to afford compound 3b $(1.002 \mathrm{~g}, 94 \%)$ as an off-white solid.

${ }^{1} \mathrm{H}$ NMR $\left(400 \mathrm{MHz}, \mathrm{CDCl}_{3}\right)$ (rotamers observed) $\delta=7.48-7.37(\mathrm{~m}, 2 \mathrm{H}), 7.36-7.31(\mathrm{~m}, 2 \mathrm{H}), 7.26-$ $7.22(\mathrm{~m}, 1 \mathrm{H}), 6.14-6.05(\mathrm{~m}, 2 \mathrm{H}), 1.59-1.50(\mathrm{~m}, 18 \mathrm{H}) \mathrm{ppm}$.

${ }^{13} \mathrm{C}$ NMR $\left(100 \mathrm{MHz}, \mathrm{CDCl}_{3}\right)$ (rotamers observed) $\delta=150.7,128.8,128.7,127.3,127.2,126.2$, $125.6,115.8,114.9,83.9,83.7,82.9,82.7,28.3,28.2,28.2,27.1 \mathrm{ppm}$.

HRMS $\left(\mathrm{ES}^{+}\right): \mathrm{m} / \mathrm{z}[\mathrm{M}+\mathrm{Na}]^{+}$Calcd for $\mathrm{C}_{18} \mathrm{H}_{24} \mathrm{Cl}_{2} \mathrm{~N}_{2} \mathrm{O}_{4} \mathrm{Na}^{+}$425.1011; Found 425.1005.

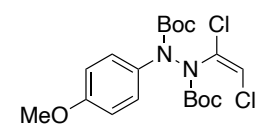

di-tert-butyl (E)-1-(1,2-dichlorovinyl)-2-(4-methoxyphenyl)hydrazine-1,2-dicarboxylate (3c) A flask was charged with sodium hydride (60\% dispersion in mineral oil) $(1.55 \mathrm{~g}, 38.81 \mathrm{mmol}, 2.1$ equiv), washed with hexanes, and dried under a stream of $\mathrm{N}_{2}$. A solution of 2c $(6.25 \mathrm{~g}, 18.48$ mmol, 1.0 equiv) in DMF (75 mL) was added in one portion and stirred at ambient temperature for $1 \mathrm{~h}$. Trichloroethylene ( $1.75 \mathrm{~mL}, 19.40 \mathrm{mmol}, 1.05$ equiv) was added dropwise over $1 \mathrm{~h}$ and allowed to stir for an additional $1 \mathrm{~h}$. The solution was poured into water $(1000 \mathrm{~mL})$ and extracted with EtOAc $(3 \times 400 \mathrm{~mL})$. The organic extracts were combined, washed with brine $(2 \times 600 \mathrm{~mL})$, dried over $\mathrm{Na}_{2} \mathrm{SO}_{4}$, filtered, and concentrated under reduced pressure. The residue was purified by chromatography on silica gel (5\% EtOAc in hexanes) to afford compound $3 \mathrm{c}(8.01 \mathrm{~g},>99 \%)$ as a pale oil.

${ }^{1} \mathrm{H}$ NMR $\left(400 \mathrm{MHz}, \mathrm{CDCl}_{3}\right)$ (rotamers observed) $\delta=7.40-7.28(\mathrm{~m}, 2 \mathrm{H}), 6.88-6.81(\mathrm{~m}, 2 \mathrm{H}), 6.16-$ $6.04(\mathrm{~m}, 1 \mathrm{H}), 3.80-3.76(\mathrm{~m}, 3 \mathrm{H}), 1.60-1.38(\mathrm{~m}, 18 \mathrm{H}) \mathrm{ppm}$.

${ }^{13} \mathrm{C}$ NMR $\left(100 \mathrm{MHz} \mathrm{CDCl}_{3}\right)$ (rotamers observed) $\delta=158.8,150.6,150.1,130.0,128.3,127.6$, 116.1, 115.1, 114.0, 83.7, 83.5, 82.4, 55.5, 28.3, 28.2, 28.2, $28.1 \mathrm{ppm}$.

HRMS $\left(\mathrm{ES}^{+}\right): \mathrm{m} / \mathrm{z}[\mathrm{M}+\mathrm{Na}]^{+}$Calcd for $\mathrm{C}_{19} \mathrm{H}_{26} \mathrm{Cl}_{2} \mathrm{~N}_{2} \mathrm{O}_{5} \mathrm{Na}^{+} 455.1116$; Found 455.1116. 
Boc

di-tert-butyl dicarboxylate (3d)

A flask was charged with sodium hydride (60\% dispersion in mineral oil) $(0.036 \mathrm{~g}, 0.897 \mathrm{mmol}$, 2.1 equiv), washed with hexanes, and dried under a stream of $\mathrm{N}_{2}$. A solution of $2 \mathbf{d}(0.150 \mathrm{~g}, 0.427$ mmol, 1.0 equiv) in DMF $(1.7 \mathrm{~mL})$ was added in one portion and stirred at ambient temperature for $1 \mathrm{~h}$. Trichloroethylene $(0.04 \mathrm{~mL}, 0.448 \mathrm{mmol}, 1.05$ equiv) was added dropwise over $1 \mathrm{~h}$ and allowed to stir for an additional $1 \mathrm{~h}$. The solution was poured into water $(50 \mathrm{~mL})$ and extracted with EtOAc $(3 \times 20 \mathrm{~mL})$. The organic extracts were combined, washed with brine $(50 \mathrm{~mL})$, dried over $\mathrm{Na}_{2} \mathrm{SO}_{4}$, filtered, and concentrated under reduced pressure. The residue was purified by chromatography on silica gel (5\% EtOAc in hexanes) to afford compound $\mathbf{3 d}(0.162 \mathrm{~g}, 85 \%)$ as a white semi-solid.

${ }^{1} \mathrm{H}$ NMR $\left(400 \mathrm{MHz}, \mathrm{CDCl}_{3}\right)$ (rotamers observed) $\delta=7.30-7.18(\mathrm{~m}, 2 \mathrm{H}), 6.68-6.62(\mathrm{~m}, 2 \mathrm{H}), 6.14-$ $6.04(\mathrm{~m}, 1 \mathrm{H}), 2.94-2.92(\mathrm{~m}, 6 \mathrm{H}), 1.60-1.38(\mathrm{~m}, 18 \mathrm{H}) \mathrm{ppm}$.

${ }^{13} \mathrm{C}$ NMR $\left(100 \mathrm{MHz}, \mathrm{CDCl}_{3}\right.$ ) (rotamers observed) $\delta=153.5,150.5,150.2,150.0,130.0,127.9$, $127.3,115.9,115.0,112.4,84.2,83.4,83.2,82.2,81.9,40.6,28.2,28.2,28.1,28.1,28.0,28.0$ ppm.

HRMS $\left(\mathrm{ES}^{+}\right): \mathrm{m} / \mathrm{z}[\mathrm{M}+\mathrm{H}]^{+}$Calcd for $\mathrm{C}_{20} \mathrm{H}_{30} \mathrm{Cl}_{2} \mathrm{~N}_{3} \mathrm{O}_{4}{ }^{+}$446.1613: Found 446.1620.

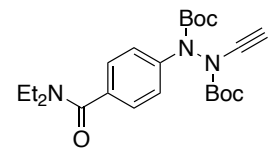

di-tert-butyl 1-(4-(diethylcarbamoyl)phenyl)-2-ethynylhydrazine-1,2-dicarboxylate (4a)

To a $-78^{\circ} \mathrm{C}$ cooled solution of compound $3 \mathrm{a}(0.500 \mathrm{~g}, 1.0 \mathrm{mmol}, 1.0$ equiv) in THF (4 mL) was added $n$-BuLi (2.5 M in hexanes) $(0.44 \mathrm{~mL}, 1.1 \mathrm{mmol}, 1.1$ equiv) dropwise over $10 \mathrm{~min}$, after which the solution was allowed to warm slowly to ambient temperature and stirred for $1 \mathrm{~h}$. The solution was then cooled to $-78^{\circ} \mathrm{C}, n$-BuLi $(2.5 \mathrm{M}$ in hexanes) $(0.4 \mathrm{~mL}, 1.0 \mathrm{mmol}, 1.0$ equiv) was added dropwise over $5 \mathrm{~min}$, and the reaction stirred for $15 \mathrm{~min}$. The solution was slowly quenched with $\mathrm{H}_{2} \mathrm{O}(10 \mathrm{~mL})$, while warming to ambient temperature. The solution was extracted with $\mathrm{Et}_{2} \mathrm{O}(3 \mathrm{x}$ $10 \mathrm{~mL}$ ), dried over $\mathrm{Na}_{2} \mathrm{SO}_{4}$, filtered, and concentrated under reduced pressure. The residue was purified by chromatography on silica gel (15\% EtOAc in hexanes to $30 \%$ EtOAc in hexanes) to afford compound $4 \mathrm{a}(0.151 \mathrm{~g}, 35 \%)$ as a brown oil.

${ }^{1} \mathrm{H}$ NMR $\left(400 \mathrm{MHz}, \mathrm{CDCl}_{3}\right)$ (rotamers observed) $\delta=7.49-7.40(\mathrm{~m}, 2 \mathrm{H}), 7.38-7.30(\mathrm{~m}, 2 \mathrm{H}), 3.56-$ $3.40(\mathrm{~m}, 2 \mathrm{H}), 3.36-3.20(\mathrm{~m}, 2 \mathrm{H}), 3.08-3.02(\mathrm{~m}, 1 \mathrm{H}), 1.61-1.32(\mathrm{~m}, 18 \mathrm{H}), 1.27-1.15(\mathrm{~m}, 3 \mathrm{H})$, $1.15-1.01(\mathrm{~m}, 3 \mathrm{H}) \mathrm{ppm}$.

${ }^{13} \mathrm{C}$ NMR (100 MHz, $\mathrm{CDCl}_{3}$ ) (rotamers observed) $\delta=170.7,152.2,151.9,151.8,151.5,141.0$, $135.0,127.5,127.1,122.8,84.9,83.5,83.4,75.1,61.4,60.7,43.4,39.4,29.8,28.2,28.0,28.0$, 14.3, $13.0 \mathrm{ppm}$.

HRMS (ES $): m / z[M+H]^{+}$Calcd for $\mathrm{C}_{23} \mathrm{H}_{34} \mathrm{~N}_{3} \mathrm{O}_{5}{ }^{+}$432.2498; Found 432.2513.

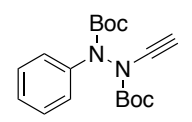

di-tert-butyl 1-ethynyl-2-phenylhydrazine-1,2-dicarboxylate (4b) 
To a $-78{ }^{\circ} \mathrm{C}$ cooled solution of compound $3 \mathrm{~b}(0.300 \mathrm{~g}, 0.744 \mathrm{mmol}, 1.0$ equiv) in THF $(3.0 \mathrm{~mL})$ was added $n$-BuLi $(2.5 \mathrm{M}$ in hexanes) $(0.33 \mathrm{~mL}, 0.818 \mathrm{mmol}, 1.1$ equiv) dropwise over $10 \mathrm{~min}$, after which the solution was allowed to warm slowly to ambient temperature and stirred for $1 \mathrm{~h}$. The solution was cooled to $-78^{\circ} \mathrm{C}, n$-BuLi $(2.5 \mathrm{M}$ in hexanes) $(0.30 \mathrm{~mL}, 0.744 \mathrm{~mol}, 1.0$ equiv) was added dropwise over $5 \mathrm{~min}$, and the reaction stirred for $15 \mathrm{~min}$. The solution was slowly quenched with (1:1) $\mathrm{Et}_{2} \mathrm{O} / \mathrm{H}_{2} \mathrm{O}(3 \mathrm{~mL})$ while warming to ambient temperature. An additional portion of $\mathrm{H}_{2} \mathrm{O}$ $(15 \mathrm{~mL})$ was added and the layers were separated. The aqueous layer was further extracted with $\mathrm{Et}_{2} \mathrm{O}(2 \times 15 \mathrm{~mL})$. The organic extracts were combined, washed with brine $(15 \mathrm{~mL})$, dried over $\mathrm{Na}_{2} \mathrm{SO}_{4}$, filtered, and concentrated under reduced pressure. The residue was purified by chromatography on silica gel (5\% EtOAc in hexanes) to afford compound $\mathbf{4 b}(0.176 \mathrm{~g}, 71 \%)$ as a brown oil.

${ }^{1} \mathrm{H}$ NMR $\left(600 \mathrm{MHz}, \mathrm{CDCl}_{3}\right)$ (rotamers observed) $\delta=7.49-7.40(\mathrm{~m}, 2 \mathrm{H}), 7.35(\mathrm{t}, \mathrm{J}=5.0 \mathrm{~Hz}, 2 \mathrm{H})$, 7.26-7.21 (m, 1H), 3.10-3.02 (m, 1H), 1.56-1.44 (m, 18H) ppm.

${ }^{13} \mathrm{C}$ NMR $\left(150 \mathrm{MHz}, \mathrm{CDCl}_{3}\right.$ ) (rotamers observed) $\delta=152.1,140.3,128.9,126.7,124.0,84.6$, 83.1, 75.4, 61.2, 60.7, 28.3, 28.0 ppm.

HRMS (ES $): m / z[2 \mathrm{M}+\mathrm{Na}]^{+}$Calcd for $\mathrm{C}_{36} \mathrm{H}_{48} \mathrm{~N}_{4} \mathrm{O}_{8} \mathrm{Na}^{+} 687.3370$; Found 687.3399.

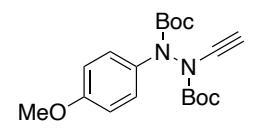

di-tert-butyl 1-ethynyl-2-(4-methoxyphenyl)hydrazine-1,2-dicarboxylate (4c)

To a $-78{ }^{\circ} \mathrm{C}$ cooled solution of compound $3 \mathrm{c}(1.16 \mathrm{~g}, 2.68 \mathrm{mmol}, 1.0$ equiv) in THF $(10.8 \mathrm{~mL})$ was added $n$-BuLi (2.5 M in hexanes) $(1.18 \mathrm{~mL}, 2.95 \mathrm{mmol}, 1.1$ equiv) dropwise over $10 \mathrm{~min}$, after which the solution was allowed to warm slowly to ambient temperature and the reaction stirred for $1 \mathrm{~h}$. The solution was cooled to $-78^{\circ} \mathrm{C}, n$-BuLi $(2.5 \mathrm{M}$ in hexanes) $(1.07 \mathrm{~mL}, 2.68 \mathrm{~mol}, 1.0$ equiv) was added dropwise over $5 \mathrm{~min}$, and the reaction stirred for $15 \mathrm{~min}$. The solution was slowly quenched with $\mathrm{H}_{2} \mathrm{O}(25 \mathrm{~mL})$ while warming to ambient temperature. An additional portion of $\mathrm{H}_{2} \mathrm{O}(400 \mathrm{~mL})$ was added the solution was extracted with EtOAc $(3 \times 200 \mathrm{~mL})$. The organic extracts were combined, washed with brine $(200 \mathrm{~mL})$, dried over $\mathrm{Na}_{2} \mathrm{SO}_{4}$, filtered, and concentrated under reduced pressure. The residue was purified by chromatography on silica gel (8\% EtOAc in hexanes) to afford compound $4 \mathrm{c}(0.815 \mathrm{~g}, 84 \%)$ as a brown oil.

${ }^{1} \mathrm{H}$ NMR $\left(400 \mathrm{MHz}, \mathrm{CDCl}_{3}\right)$ (rotamers observed) $\delta 7.41-7.30(\mathrm{~m}, 2 \mathrm{H}), 6.87(\mathrm{~d}, J=8.8 \mathrm{~Hz}, 2 \mathrm{H})$, $3.80(\mathrm{~s}, 3 \mathrm{H}), 3.10-3.04(\mathrm{~m}, 1 \mathrm{H}), 1.56-1.44(\mathrm{~m}, 18 \mathrm{H}) \mathrm{ppm}$.

${ }^{13} \mathrm{C}$ NMR $\left(100 \mathrm{MHz}, \mathrm{CDCl}_{3}\right)$ (rotamers observed) $\delta=158.7,152.0,133.3,127.2,126.6,114.1$, 84.5, 82.7, 75.5, 61.3, 60.7, 55.6, 28.3, 28.2, 28.1, 28.0 ppm.

HRMS (ES $\left.{ }^{+}\right): m / z[M+N a]^{+} \mathrm{C}_{19} \mathrm{H}_{26} \mathrm{~N}_{2} \mathrm{O}_{5} \mathrm{Na}^{+}$Calcd for 385.1739; Found 385.1746.

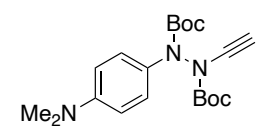

di-tert-butyl 1-(4-(dimethylamino)phenyl)-2-ethynylhydrazine-1,2-dicarboxylate (4d)

To a $-78{ }^{\circ} \mathrm{C}$ cooled solution of compound $3 \mathbf{d}(0.130 \mathrm{~g}, 0.291 \mathrm{mmol}, 1.0$ equiv $)$ in THF $(1.2 \mathrm{~mL})$ was added $n$-BuLi (2.5 M in hexanes) $(0.128 \mathrm{~mL}, 0.32 \mathrm{mmol}, 1.1$ equiv) dropwise over $10 \mathrm{~min}$, after which the solution was allowed to warm slowly to ambient temperature and the reaction stirred for $1 \mathrm{~h}$. The solution was cooled to $-78^{\circ} \mathrm{C}, n$-BuLi $(2.5 \mathrm{M}$ in hexanes) $(0.116 \mathrm{~mL}, 0.291$ mmol, 1.0 equiv) was added dropwise over $5 \mathrm{~min}$, and the reaction stirred for $15 \mathrm{~min}$. The solution was slowly quenched with $\mathrm{H}_{2} \mathrm{O}(10 \mathrm{~mL})$ while warming to ambient temperature. The solution was 
extracted with $\mathrm{Et}_{2} \mathrm{O}(3 \times 10 \mathrm{~mL})$. The organic extracts were combined, washed with brine $(10 \mathrm{~mL})$, dried over $\mathrm{Na}_{2} \mathrm{SO}_{4}$, filtered, and concentrated under reduced pressure. The residue was purified by chromatography on silica gel (10\% EtOAC in hexanes) to afford compound $\mathbf{4 d}(0.079 \mathrm{~g}, 72 \%)$ as a brown oil.

${ }^{1} \mathrm{H}$ NMR $\left(400 \mathrm{MHz}, \mathrm{CDCl}_{3}\right)$ (rotamers observed) $\delta=7.38-7.22(\mathrm{~m}, 2 \mathrm{H}), 6.66(\mathrm{~d}, \mathrm{~J}=8.6 \mathrm{~Hz}, 2 \mathrm{H})$, 3.10-3.02 (m, 1H), 2.96-2.89 (m, 6H), 1.56-1.40 (m, 18H) ppm.

${ }^{13} \mathrm{C}$ NMR (100 MHz, $\mathrm{CDCl}_{3}$ ) (rotamers observed) $\delta=152.9,152.5,152.0,149.9,127.2,126.7$, 115.5, 112.3, 93.0, 84.1, 82.2, 75.6, 61.1, 60.6, 41.7, 40.6, 28.4, 28.3, 28.3, 28.0 ppm.

HRMS $\left(\mathrm{ES}^{+}\right): \mathrm{m} / \mathrm{z}[\mathrm{M}+\mathrm{H}]^{+}$Calcd for $\mathrm{C}_{20} \mathrm{H}_{30} \mathrm{~N}_{3} \mathrm{O}_{4}{ }^{+}$376.2236; Found 376.2234.

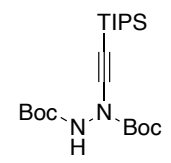

di-tert-butyl 1-((triisopropylsilyl)ethynyl)hydrazine-1,2-dicarboxylate (5)

To a $-78^{\circ} \mathrm{C}$ cooled solution of triisopropylsilyl acetylene $(1.218 \mathrm{~mL}, 5.429 \mathrm{mmol}, 1.0$ equiv) in THF $(18 \mathrm{~mL})$ was added $n$-BuLi $(2.5 \mathrm{M}$ in hexanes) $(2.39 \mathrm{~mL}, 5.97 \mathrm{mmol}, 1.1$ equiv) dropwise. The resulting solution was stirred for $30 \mathrm{~min}$ at $-78^{\circ} \mathrm{C}$. A solution of di-tert-butyl azodicarboxylate (1.5 $\mathrm{g}, 6.514 \mathrm{mmol}, 1.2$ equiv) in THF (9 mL) was slowly added before letting the solution warm to ambient temperature. The solution was stirred for $2 \mathrm{~h}$, then poured into $\mathrm{H}_{2} \mathrm{O}(100 \mathrm{~mL})$ and extracted with EtOAc $(3 \times 100 \mathrm{~mL})$. The organic extracts were combined, washed with brine $(100$ $\mathrm{mL}$ ), dried over $\mathrm{Na}_{2} \mathrm{SO}_{4}$, filtered, and concentrated under reduced pressure. The residue was purified by chromatography on silica gel (4\% EtOAc in hexanes to $10 \%$ EtOAc in hexanes) to afford compound $5(1.082 \mathrm{~g}, 48 \%)$ as a clear oil.

${ }^{1} \mathrm{H}$ NMR $\left(400 \mathrm{MHz} \mathrm{CDCl}_{3}\right) \delta=6.66(\mathrm{~s}, 1 \mathrm{H}), 1.49(\mathrm{~s}, 9 \mathrm{H}), 1.47(\mathrm{~s}, 9 \mathrm{H}), 1.06(\mathrm{~m}, 21 \mathrm{H}) \mathrm{ppm}$.

${ }^{13} \mathrm{C}$ NMR $\left(100 \mathrm{MHz}, \mathrm{CDCl}_{3}\right) \delta=154.0,152.9,96.2,84.2,82.3,69.8,28.2,28.1,18.7,11.4 \mathrm{ppm}$. HRMS $\left(\mathrm{ES}^{+}\right): \mathrm{m} / \mathrm{z}[\mathrm{M}+\mathrm{Na}]^{+}$calcd for $\mathrm{C}_{21} \mathrm{H}_{40} \mathrm{~N}_{2} \mathrm{O}_{4} \mathrm{SiNa}^{+} 435.2655$, found 435.2651.

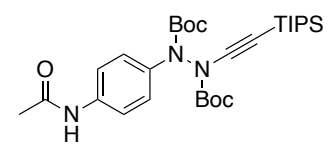

\section{di-tert-butyl} dicarboxylate (6)

1-(4-acetamidophenyl)-2-((triisopropylsilyl)ethynyl)hydrazine-1,2A Schlenk tube was sequentially charged with compound 5 ( $0.770 \mathrm{~g}, 1.87 \mathrm{mmol}, 1.0$ equiv), $\mathrm{Cs}_{2} \mathrm{CO}_{3}$ (0.730 g, $2.24 \mathrm{mmol}, 1.2$ equiv), 1,10-phenanthroline (0.067 g, $0.374 \mathrm{mmol}, 0.2$ equiv), copper(I) iodide ( $0.356 \mathrm{~g}, 1.87 \mathrm{mmol}, 1.0$ equiv), $\mathrm{N}$-(4-iodophenyl) acetamide $(0.584 \mathrm{~g}, 2.24 \mathrm{mmol}$, 1.2 equiv), and DMF $(7.4 \mathrm{~mL})$. The mixture was stirred at $90^{\circ} \mathrm{C}$ for $48 \mathrm{~h}$, then cooled to ambient temperature, diluted with EtOAc $(150 \mathrm{~mL})$, and filtered through celite. The reaction vessel and filter cake were washed with additional EtOAc. The filtrate was poured into water $(400 \mathrm{~mL})$ and the layers were separated. The aqueous layer was further extracted with EtOAc $(2 \times 150 \mathrm{~mL})$. The organic extracts were combined, washed with brine $(400 \mathrm{~mL})$, dried over $\mathrm{Na}_{2} \mathrm{SO}_{4}$, filtered, and concentrated under reduced pressure. The residue was purified by chromatography on silica gel (40\% EtOAc in hexanes) to afford compound $6(0.470 \mathrm{~g}, 46 \%)$ as an off-white semi-solid.

${ }^{1} \mathrm{H}$ NMR $\left(400 \mathrm{MHz}, \mathrm{CDCl}_{3}\right) \delta=8.12(\mathrm{~s}, 1 \mathrm{H}), 7.41-7.32(\mathrm{~m}, 4 \mathrm{H}), 2.06(\mathrm{~s}, 3 \mathrm{H}), 1.54-1.46(\mathrm{~m}, 18 \mathrm{H})$, $1.08-1.03(\mathrm{~m}, 21 \mathrm{H}) \mathrm{ppm}$.

${ }^{13} \mathrm{C}$ NMR $\left(100 \mathrm{MHz}, \mathrm{CDCl}_{3}\right) \delta=168.8,152.8,152.1,137.4,135.8,126.0,120.4,95.6,84.2,82.8$, 71.2, 28.2, 28.1, 24.3, 18.7, $11.3 \mathrm{ppm}$.

HRMS (ES $\left.{ }^{+}\right): m / z[M+N a]^{+}$Calcd for $\mathrm{C}_{29} \mathrm{H}_{47} \mathrm{~N}_{3} \mathrm{O}_{5} \mathrm{SiNa}^{+}$568.3183; Found 568.3206. 


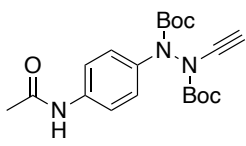

di-tert-butyl 1-(4-acetamidophenyl)-2-ethynylhydrazine-1,2-dicarboxylate (4e)

To a $0^{\circ} \mathrm{C}$ cooled solution of compound $6(0.450 \mathrm{~g}, 0.824 \mathrm{mmol}, 1.0$ equiv) in THF $(8.24 \mathrm{~mL})$ was added tetrabutylammonium fluoride (1M in THF) $(1.65 \mathrm{~mL}, 1.65 \mathrm{mmol}, 2.0$ equiv) dropwise, and the reaction was stirred for $30 \mathrm{~min}$. The solution was warmed to ambient temperature, poured into $\mathrm{H}_{2} \mathrm{O}(100 \mathrm{~mL})$, and extracted with EtOAc $(3 \times 50 \mathrm{~mL})$. The organic extracts were combined, washed with brine $(50 \mathrm{~mL})$, dried over $\mathrm{Na}_{2} \mathrm{SO}_{4}$, filtered, and concentrated under reduced pressure. The residue was purified by chromatography on silica gel (40\% EtOAc in hexanes) to afford compound $4 \mathrm{e}(0.314 \mathrm{~g}, 98 \%)$ as a brown oil.

${ }^{1} \mathrm{H}$ NMR $\left(400 \mathrm{MHz}, \mathrm{CDCl}_{3}\right)$ (rotamers observed $\delta=7.86(\mathrm{~s}, 1 \mathrm{H}), 7.46-7.32(\mathrm{~m}, 4 \mathrm{H}), 3.08-3.05$ $(\mathrm{m}, 1 \mathrm{H}), 2.11(\mathrm{~s}, 3 \mathrm{H}), 1.50(\mathrm{~s}, 18 \mathrm{H}) \mathrm{ppm}$.

${ }^{13} \mathrm{C}$ NMR $\left(100 \mathrm{MHz}, \mathrm{CDCl}_{3}\right.$ ) (rotamers observed) $\delta=168.7,152.4,152.1,137.1,135.9,129.0$, 125.6, 120.2, 84.8, 83.1, 75.2, 61.5, 60.9, 28.3, 28.0, $24.5 \mathrm{ppm}$.

HRMS $\left(\mathrm{ES}^{+}\right): \mathrm{m} / \mathrm{z}[2 \mathrm{M}+\mathrm{Na}]^{+}$Calcd for $\mathrm{C}_{40} \mathrm{H}_{54} \mathrm{~N}_{6} \mathrm{O}_{10} \mathrm{Na}^{+} 801.3799$; Found 801.3810.

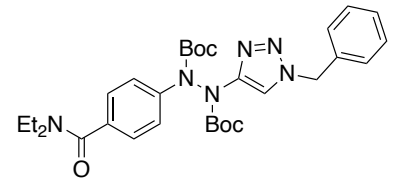

di-tert-butyl 1-(1-benzyl-1H-1,2,3-triazol-4-yl)-2-(4-(diethylcarbamoyl)phenyl)hydrazine-1,2dicarboxylate (7a)

To a solution of arylynehydrazide $4 \mathrm{a}(0.100 \mathrm{~g}, 0.232 \mathrm{mmol}, 1.0$ equiv) in THF $(0.5 \mathrm{~mL})$ was added benzyl azide ${ }^{3}(0.037 \mathrm{~g}, 0.278 \mathrm{mmol}, 1.2$ equiv), triethylamine $(0.04 \mathrm{~mL}, 0.278 \mathrm{mmol}, 1.2$ equiv), and copper(I) iodide (0.009 $\mathrm{g}, 0.05 \mathrm{mmol}, 0.2$ equiv). The mixture was stirred at ambient temperature for $24 \mathrm{~h}$, diluted with EtOAc $(10 \mathrm{~mL})$, and filtered through celite. The reaction vessel and filter cake were washed with additional EtOAc. The organic solution was concentrated under reduced pressure and purified by chromatography on silica gel (40\% EtOAc in hexanes) to afford compound $7 \mathrm{a}(0.096 \mathrm{~g}, 74 \%)$ as a white solid.

${ }^{1} \mathrm{H}$ NMR $\left(400 \mathrm{MHz}, \mathrm{CDCl}_{3}\right)$ (rotamers observed) $\delta=7.78-7.66(\mathrm{~m}, 1 \mathrm{H}), 7.62-7.46(\mathrm{~m}, 2 \mathrm{H}), 7.36-$ $7.22(\mathrm{~m}, 6 \mathrm{H}), 5.52-5.36(\mathrm{~m}, 2 \mathrm{H}), 3.56-3.39(\mathrm{~m}, 2 \mathrm{H}), 3.36-3.17(\mathrm{~m}, 2 \mathrm{H}), 1.55-1.28(\mathrm{~m}, 18 \mathrm{H})$, $1.26-1.14(\mathrm{~m}, 3 \mathrm{H}), 1.14-1.00(\mathrm{~m}, 3 \mathrm{H}) \mathrm{ppm}$.

${ }^{13} \mathrm{C}$ NMR $\left(100 \mathrm{MHz}_{\mathrm{CDCl}}\right.$ ) (rotamers observed) $\delta=171.0,152.2,146.4,134.5,129.2,128.9$, $128.2,126.8,122.6,113.5,83.9,83.4,82.5,82.4,54.8,43.4,39.4,28.2,14.3,13.0$ ppm.

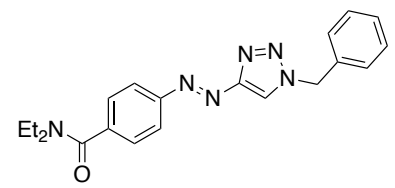

(E)-4-((1-benzyl-1H-1,2,3-triazol-4-yl)diazenyl)-N,N-diethylbenzamide (8a)

To a $0^{\circ} \mathrm{C}$ cooled solution of compound $7 \mathrm{a}(0.080 \mathrm{~g}, 0.142 \mathrm{mmol}, 1.0$ equiv) in $\mathrm{DCM}(7 \mathrm{~mL})$ was added $\mathrm{N}$-methylmorpholine $(0.08 \mathrm{~mL}, 0.71 \mathrm{mmol}, 5.0$ equiv $)$, followed by the dropwise addition of trimethylsilyl iodide $(0.061 \mathrm{~mL}, 0.425 \mathrm{mmol}, 3.0$ equiv). The resulting solution was warmed to ambient temperature and stirred for $4 \mathrm{~h}$. The reaction vessel atmosphere was exchanged to $\mathrm{O}_{2}$. To the solution was added a saturated aqueous $\mathrm{NaHCO}_{3}$ solution $(3.5 \mathrm{~mL})$. The biphasic solution 
was stirred vigorously for $4 \mathrm{~h}$. To the solution was added DCM $(10 \mathrm{~mL})$ and $\mathrm{H}_{2} \mathrm{O}(10 \mathrm{~mL})$. The layers were separated, and the aqueous layer was further extracted with DCM $(2 \times 10 \mathrm{~mL})$. The organic extracts were combined, washed with brine $(10 \mathrm{~mL})$, dried over $\mathrm{Na}_{2} \mathrm{SO}_{4}$, filtered, and concentrated under reduced pressure. The residue was purified by chromatography on silica gel (15\% EtOAc in DCM to 30\% EtOAc in DCM) to afford compound $8 \mathbf{a}(0.017 \mathrm{~g}, 34 \%)$ as a yellow solid.

${ }^{1} \mathrm{H}$ NMR $\left(400 \mathrm{MHz}, \mathrm{CDCl}_{3}\right) \delta=7.92(\mathrm{~d}, J=8.4 \mathrm{~Hz}, 2 \mathrm{H}), 7.87(\mathrm{~s}, 1 \mathrm{H}), 7.49(\mathrm{~d}, J=8.4 \mathrm{~Hz}, 2 \mathrm{H})$, 7.44-7.30 (m, 5H), $5.60(\mathrm{~s}, 2 \mathrm{H}), 3.62-3.43(\mathrm{~m}, 2 \mathrm{H}), 3.41-3.22(\mathrm{~m}, 2 \mathrm{H}), 1.30-1.19(\mathrm{~m}, 3 \mathrm{H}), 1.19-$ $1.04(\mathrm{~m}, 3 \mathrm{H}) \mathrm{ppm}$.

${ }^{13} \mathrm{C}$ NMR $\left(100 \mathrm{MHz} \mathrm{CDCl}_{3}\right) \delta=170.4,160.8,152.7,140.3,134.0,129.5,128.5,127.4,123.2$, $116.9,115.3,55.1,43.4,39.5,14.4,13.0 \mathrm{ppm}$.

HRMS $\left(\mathrm{ES}^{+}\right): \mathrm{m} / \mathrm{z}[\mathrm{M}+\mathrm{Na}]^{+}$Calcd for $\mathrm{C}_{20} \mathrm{H}_{22} \mathrm{~N}_{6} \mathrm{ONa}^{+}$385.1753; Found 385.1745.

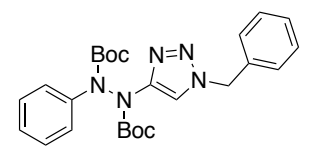

di-tert-butyl 1-(1-benzyl-1 H-1,2,3-triazol-4-yl)-2-phenylhydrazine-1,2-dicarboxylate (7b)

To a solution of arylynehydrazide $4 \mathbf{b}(0.132 \mathrm{~g}, 0.396 \mathrm{mmol}, 1.0$ equiv) in THF $(0.8 \mathrm{~mL})$ was added benzyl azide ${ }^{3}(0.063 \mathrm{~g}, 0.476 \mathrm{mmol}, 1.2$ equiv), triethylamine $(0.066 \mathrm{~mL}, 0.476 \mathrm{mmol}, 1.2$ equiv), and copper $(\mathrm{I})$ iodide $(0.015 \mathrm{~g}, 0.079 \mathrm{mmol}, 0.2$ equiv). The mixture was stirred at ambient temperature for $38 \mathrm{~h}$, diluted with $\mathrm{H}_{2} \mathrm{O}(30 \mathrm{~mL})$, and extracted with EtOAc $(3 \times 40 \mathrm{~mL})$. The organic extracts were combined, washed with brine $(50 \mathrm{~mL})$, dried over $\mathrm{Na}_{2} \mathrm{SO}_{4}$, filtered, and concentrated under reduced pressure. The residue was purified by chromatography on silica gel $(17 \%$ EtOAc in hexanes) to afford compound $7 \mathbf{b}(0.177 \mathrm{~g}, 96 \%)$ as a light yellow semi-solid.

${ }^{1} \mathrm{H}$ NMR $\left(400 \mathrm{MHz}, \mathrm{CDCl}_{3}\right)$ (rotamers observed) $\delta=7.76-7.63(\mathrm{~m}, 1 \mathrm{H}), 7.60-7.47(\mathrm{~m}, 2 \mathrm{H}), 7.38-$ $7.20(\mathrm{~m}, 6 \mathrm{H}), 7.18-7.11(\mathrm{~m}, 1 \mathrm{H}), 5.50-5.32(\mathrm{~m}, 2 \mathrm{H}), 1.52-1.29(\mathrm{~m}, 18 \mathrm{H}) \mathrm{ppm}$.

${ }^{13} \mathrm{C}$ NMR $\left(100 \mathrm{MHz}, \mathrm{CDCl}_{3}\right)$ (rotamers observed) $\delta=152.6,146.6,129.2,128.8,128.6,126.0$, $125.4,123.9,113.5,83.2,54.8,28.2 \mathrm{ppm}$.

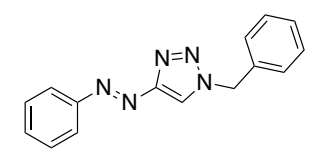

(E)-1-benzyl-4-(phenyldiazenyl)-1H-1,2,3-triazole (8b)

To a $0^{\circ} \mathrm{C}$ cooled solution of compound $7 \mathrm{~b}(0.089 \mathrm{~g}, 0.192 \mathrm{mmol}, 1.0$ equiv) in DCM $(9.6 \mathrm{~mL})$ was added $\mathrm{N}$-methylmorpholine $(0.105 \mathrm{~mL}, 0.959 \mathrm{mmol}, 5.0$ equiv), followed by the dropwise addition of trimethylsilyl iodide $(0.082 \mathrm{~mL}, 0.575 \mathrm{mmol}, 3.0$ equiv). The resulting solution was warmed to ambient temperature and stirred for $2 \mathrm{~h}$. The reaction vessel atmosphere was exchanged to $\mathrm{O}_{2}$. To the solution was added a saturated aqueous $\mathrm{NaHCO}_{3}$ solution $(4.8 \mathrm{~mL})$. The biphasic solution was stirred vigorously for $15 \mathrm{~h}$. To the solution was added DCM $(10 \mathrm{~mL})$ and $\mathrm{H}_{2} \mathrm{O}(10 \mathrm{~mL})$. The layers were separated, and the aqueous layer was further extracted with DCM $(2 \times 10 \mathrm{~mL})$. The organic extracts were combined, washed with brine $(20 \mathrm{~mL})$, dried over $\mathrm{Na}_{2} \mathrm{SO}_{4}$, filtered and concentrated under reduced pressure. The residue was purified by chromatography on silica gel (DCM) to afford compound $8 \mathrm{~b}(0.023 \mathrm{~g}, 46 \%)$ as a yellow solid.

${ }^{1} \mathrm{H}$ NMR $\left(400 \mathrm{MHz}, \mathrm{CDCl}_{3}\right) \delta=7.91$ (d, $\left.J=4.8 \mathrm{~Hz}, 2 \mathrm{H}\right), 7.86(\mathrm{~s}, 1 \mathrm{H}), 7.50-7.45(\mathrm{~m}, 3 \mathrm{H}), 7.42-$ $7.36(\mathrm{~m}, 3 \mathrm{H}), 7.34(\mathrm{~d}, J=4.5 \mathrm{~Hz}, 2 \mathrm{H}), 5.59(\mathrm{~s}, 2 \mathrm{H}) \mathrm{ppm}$.

${ }^{13} \mathrm{C}$ NMR $\left(100 \mathrm{MHz}, \mathrm{CDCl}_{3}\right) \delta=160.9,152.6,134.0,131.8,129.4,129.3,129.2,128.5,123.1$, 115.0, $55.0 \mathrm{ppm}$.

HRMS (ES $): m / z[M+N a]^{+}$Calcd for $\mathrm{C}_{15} \mathrm{H}_{13} \mathrm{~N}_{5} \mathrm{Na}^{+}$286.1069; Found 286.1063. 
Boc

di-tert-butyl dicarboxylate (7c)

1-(1-benzyl-1H-1,2,3-triazol-4-yl)-2-(4-methoxyphenyl)hydrazine-1,2To a solution of arylynehydrazide $4 \mathrm{c}(0.100 \mathrm{~g}, 0.276 \mathrm{mmol}, 1.0$ equiv $)$ in THF $(0.6 \mathrm{~mL})$ was added benzyl azide ${ }^{3}(0.043 \mathrm{~g}, 0.331 \mathrm{mmol}, 1.2$ equiv), triethylamine $(0.046 \mathrm{~mL}, 0.331 \mathrm{mmol}, 1.2$ equiv), and copper(I) iodide $(0.010 \mathrm{~g}, 0.06 \mathrm{mmol}, 0.2$ equiv). The mixture was stirred at ambient temperature for $18 \mathrm{~h}$, diluted with EtOAc $(10 \mathrm{~mL})$, and filtered through celite. The reaction vessel and filter cake were washed with additional EtOAc. The organic solution was concentrated under reduced pressure and purified by chromatography on silica gel (20\% EtOAc in hexanes) to afford compound $7 \mathrm{c}(0.128 \mathrm{~g}, 94 \%)$ as an off-white semi-solid.

${ }^{1} \mathrm{H}$ NMR $\left(400 \mathrm{MHz}, \mathrm{CDCl}_{3}\right)$ (rotamers observed) $\delta=7.79-7.68(\mathrm{~m}, 1 \mathrm{H}), 7.62-7.42(\mathrm{~m}, 2 \mathrm{H}), 7.40-$ $7.23(\mathrm{~m}, 5 \mathrm{H}), 6.88-6.82(\mathrm{~m}, 2 \mathrm{H}), 5.54-5.34(\mathrm{~m}, 2 \mathrm{H}), 3.81-3.74(\mathrm{~m}, 3 \mathrm{H}), 1.60-1.34(\mathrm{~m}, 18 \mathrm{H}) \mathrm{ppm}$. ${ }^{13} \mathrm{C}$ NMR $\left(100 \mathrm{MHz}, \mathrm{CDCl}_{3}\right)$ (rotamers observed) $\delta=155.1,152.9,152.6,152.4,146.5,134.6$, $134.4,133.9,129.0,128.7,128.3,128.1,127.6,126.8,113.8,113.5,113.4,83.3,83.0,82.1$, 81.7, 55.4, 54.6, 28.2, $28.1 \mathrm{ppm}$.

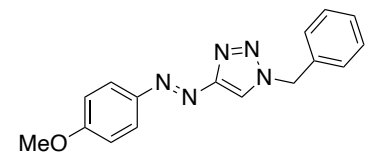

(E)-1-benzyl-4-((4-methoxyphenyl)diazenyl)-1H-1,2,3-triazole (8c)

To a $0^{\circ} \mathrm{C}$ cooled solution of compound $7 \mathrm{c}(0.100 \mathrm{~g}, 0.276 \mathrm{mmol}, 1.0$ equiv) in DCM (14 mL) was added $\mathrm{N}$-methylmorpholine $(0.152 \mathrm{~mL}, 1.38 \mathrm{mmol}, 5.0$ equiv), followed by the dropwise addition of trimethylsilyl iodide $(0.118 \mathrm{~mL}, 0.828 \mathrm{mmol}, 3.0$ equiv). The resulting solution was warmed to ambient temperature and stirred for $4 \mathrm{~h}$. The reaction vessel atmosphere was exchanged to $\mathrm{O}_{2}$. To the solution was added a saturated aqueous $\mathrm{NaHCO}_{3}$ solution $(7 \mathrm{~mL})$. The biphasic solution was stirred vigorously for $14 \mathrm{~h}$. To the solution was added DCM $(20 \mathrm{~mL})$ and $\mathrm{H}_{2} \mathrm{O}(30 \mathrm{~mL})$. The layers were separated, and the aqueous layer further extracted with DCM $(2 \times 20 \mathrm{~mL})$. The organic extracts were combined, washed with brine $(20 \mathrm{~mL})$, dried over $\mathrm{Na}_{2} \mathrm{SO}_{4}$, filtered, and concentrated under reduced pressure. The residue was purified by chromatography on silica gel $(40 \%$ hexanes in DCM to DCM) to afford compound $8 \mathrm{c}(0.052 \mathrm{~g}, 64 \%)$ as a yellow solid.

${ }^{1} \mathrm{H}$ NMR $\left(400 \mathrm{MHz}, \mathrm{CDCl}_{3}\right) \delta=7.89(\mathrm{~d}, J=9.1 \mathrm{~Hz}, 2 \mathrm{H}), 7.80(\mathrm{~s}, 1 \mathrm{H}), 7.39-7.31(\mathrm{~m}, 5 \mathrm{H}), 6.97(\mathrm{~d}$, $J=9.0 \mathrm{~Hz}, 2 \mathrm{H}), 5.57(\mathrm{~s}, 2 \mathrm{H}), 3.86(\mathrm{~s}, 3 \mathrm{H}) \mathrm{ppm}$.

${ }^{13} \mathrm{C}$ NMR $\left(100 \mathrm{MHz}, \mathrm{CDCl}_{3}\right) \delta=162.7,161.0,147.0,134.2,129.4,129.1,128.4,125.1,114.4$, $114.4 \mathrm{ppm}, 55.7,55.0 \mathrm{ppm}$.

HRMS $\left(\mathrm{ES}^{+}\right): \mathrm{m} / \mathrm{z}[\mathrm{M}+\mathrm{Na}]^{+}$Calcd for $\mathrm{C}_{16} \mathrm{H}_{15} \mathrm{~N}_{5} \mathrm{ONa}^{+}$316.1174; Found 316.1166.

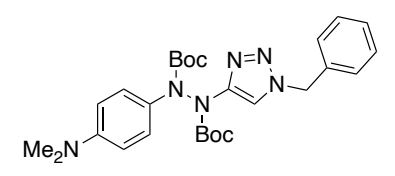

di-tert-butyl 1-(1-benzyl-1H-1,2,3-triazol-4-yl)-2-(4-(dimethylamino)phenyl)hydrazine-1,2dicarboxylate (7d)

To a solution of arylynehydrazide $4 \mathbf{d}(0.111 \mathrm{~g}, 0.296 \mathrm{mmol}, 1.0$ equiv) in THF $(0.6 \mathrm{~mL})$ was added benzyl azide ${ }^{3}(0.047 \mathrm{~g}, 0.355 \mathrm{mmol}, 1.2$ equiv), triethylamine $(0.05 \mathrm{~mL}, 0.355 \mathrm{mmol}, 1.2$ equiv), 
and copper(I) iodide $(0.011 \mathrm{~g}, 0.06 \mathrm{mmol}, 0.2$ equiv). The mixture was stirred at ambient temperature for $47 \mathrm{~h}$, diluted with EtOAc $(10 \mathrm{~mL})$, and filtered through celite. The reaction vessel and filter cake were washed with additional EtOAc. The organic solution was concentrated under reduced pressure and purified by chromatography on silica gel (20\% EtOAc in hexanes to $25 \%$ EtOAc in hexanes) to afford compound $\mathbf{7 d}(0.113 \mathrm{~g}, 75 \%)$ as a light yellow solid.

${ }^{1} \mathrm{H}$ NMR $\left(400 \mathrm{MHz}, \mathrm{CDCl}_{3}\right)$ (rotamers observed) $\delta=7.75-7.63(\mathrm{~m}, 1 \mathrm{H}), 7.53-7.46(\mathrm{~m}, 2 \mathrm{H}), 7.36-$ $7.29(\mathrm{~m}, 3 \mathrm{H}), 7.29-7.21(\mathrm{~m}, 2 \mathrm{H}), 6.65(\mathrm{t}, \mathrm{J}=9.3 \mathrm{~Hz}, 2 \mathrm{H}), 5.50-5.32(\mathrm{~m}, 2 \mathrm{H}), 2.94-2.86(\mathrm{~m}, 6 \mathrm{H})$, $1.58-1.34(\mathrm{~m}, 18 \mathrm{H}) \mathrm{ppm}$.

${ }^{13} \mathrm{C}$ NMR $\left(100 \mathrm{MHz}, \mathrm{CDCl}_{3}\right)$ (rotamers observed) $\delta=153.4,153.3,152,8,152.5,149.6,149.3$, 146.6, 146.5, 134.7, 134.5, 130.9, 130.3, 129.1, 128.7, 128.6, 128.3, 128.1, 127.8, 127.5, 127.1, $113.9,113.5,112.4,111.8,83.1,82.8,82.7,81.7,81.3,54.6,40.8,40.6,28.3,28.2$, 28.2 ppm.

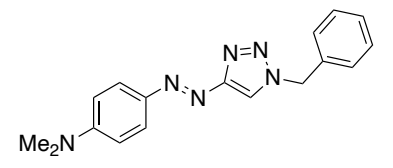

(E)-4-((1-benzyl-1H-1,2,3-triazol-4-yl)diazenyl)-N,N-dimethylaniline (8d)

To a $0^{\circ} \mathrm{C}$ cooled solution of compound $7 \mathbf{d}(0.060 \mathrm{~g}, 0.118 \mathrm{mmol}, 1.0$ equiv) in DCM $(5.9 \mathrm{~mL})$ was added $\mathrm{N}$-methylmorpholine $(0.065 \mathrm{~mL}, 0.59 \mathrm{mmol}, 5.0$ equiv), followed by the dropwise addition of trimethylsilyl iodide $(0.05 \mathrm{~mL}, 0.354 \mathrm{mmol}, 3.0$ equiv). The resulting solution was warmed to ambient temperature and stirred for $2 \mathrm{~h}$. The reaction vessel atmosphere was exchanged to $\mathrm{O}_{2}$. To the solution was added a saturated aqueous $\mathrm{NaHCO}_{3}$ solution $(2.95 \mathrm{~mL})$. The biphasic solution was stirred vigorously for $18 \mathrm{~h}$. To the solution was added DCM $(10 \mathrm{~mL})$ and $\mathrm{H}_{2} \mathrm{O}(10 \mathrm{~mL})$. The layers were separated, and the aqueous layer was further extracted with DCM $(2 \times 10 \mathrm{~mL})$. The organic extracts were combined, washed with brine $(10 \mathrm{~mL})$, dried over $\mathrm{Na}_{2} \mathrm{SO}_{4}$, filtered, and concentrated under reduced pressure. The residue was purified by chromatography on silica gel (DCM to $10 \%$ EtOAc in DCM) to afford compound $8 \mathbf{d}(0.028 \mathrm{~g}, 74 \%)$ as an orange solid.

${ }^{1} \mathrm{H}$ NMR $\left(400 \mathrm{MHz}, \mathrm{CDCl}_{3}\right) \delta=7.85(\mathrm{~d}, \mathrm{~J}=9.1 \mathrm{~Hz}, 2 \mathrm{H}), 7.73(\mathrm{~s}, 1 \mathrm{H}), 7.39-7.31(\mathrm{~m}, 5 \mathrm{H}), 6.71(\mathrm{~d}$, $J=9.2 \mathrm{~Hz}, 2 \mathrm{H}), 5.56(\mathrm{~s}, 2 \mathrm{H}), 3.07(\mathrm{~s}, 6 \mathrm{H}) \mathrm{ppm}$.

${ }^{13} \mathrm{C}$ NMR $\left(100 \mathrm{MHz}, \mathrm{CDCl}_{3}\right) \delta=161.7,152.9,143.6,134.4,129.3,129.0,128.4,125.4,113.5$, 111.5, 54.9, $40.4 \mathrm{ppm}$.

HRMS $\left(\mathrm{ES}^{+}\right): \mathrm{m} / \mathrm{z}[\mathrm{M}+\mathrm{H}]^{+}$Calcd for $\mathrm{C}_{17} \mathrm{H}_{19} \mathrm{~N}_{6}{ }^{+} 307.1671$; Found 307.1655.

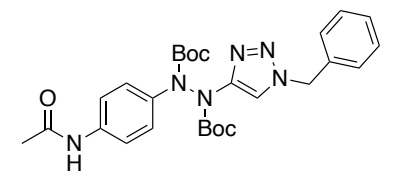

\section{di-tert-butyl}

dicarboxylate $(7 \mathrm{e})$

1-(4-acetamidophenyl)-2-(1-benzyl-1H-1,2,3-triazol-4-yl)hydrazine-1,2-

To a solution of arylynehydrazide $4 \mathrm{e}(0.076 \mathrm{~g}, 0.195 \mathrm{mmol}, 1.0$ equiv) in THF $(0.4 \mathrm{~mL})$ was added benzyl azide ${ }^{3}(0.031 \mathrm{~g}, 0.234 \mathrm{mmol}, 1.2$ equiv), triethylamine $(0.027 \mathrm{~mL}, 0.234 \mathrm{mmol}, 1.2$ equiv), and copper(l) iodide $(0.008 \mathrm{~g}, 0.04 \mathrm{mmol}, 0.2$ equiv). The mixture was stirred at ambient temperature for $24 \mathrm{~h}$, diluted with EtOAc $(10 \mathrm{~mL})$, and filtered through celite. The reaction vessel and filter cake were washed with additional EtOAc. The organic solution was concentrated under reduced pressure and purified by chromatography on silica gel (50\% EtOAc in hexanes) to afford compound $7 \mathrm{e}(0.084 \mathrm{~g}, 83 \%)$ as a white semi-solid.

${ }^{1} \mathrm{H}$ NMR $\left(400 \mathrm{MHz}, \mathrm{CDCl}_{3}\right)$ (rotamers observed) $\delta=8.04(\mathrm{~s}, 1 \mathrm{H}), 7.77-7.69(\mathrm{~m}, 1 \mathrm{H}), 7.46-7.25$ $(\mathrm{m}, 9 \mathrm{H}), 5.46-5.40(\mathrm{~m}, 2 \mathrm{H}), 2.08(\mathrm{~s}, 3 \mathrm{H}), 1.50-1.37(\mathrm{~m}, 18 \mathrm{H}) \mathrm{ppm}$. 
${ }^{13} \mathrm{C}$ NMR $\left(100 \mathrm{MHz}, \mathrm{CDCl}_{3}\right)$ (rotamers observed) $\delta=168.8,152.9,146.4,136.6,129.2,128.9$, $128.2,125.8,125.1,120.2,119.8,113.7,83.4,82.2,54.9,28.2,24.5$ ppm.

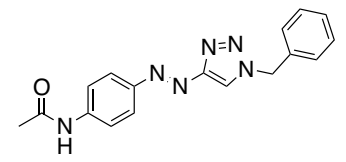

(E)-N-(4-((1-benzyl-1H-1,2,3-triazol-4-yl)diazenyl)phenyl)acetamide (8e)

To a $0^{\circ} \mathrm{C}$ cooled solution of compound $7 \mathrm{e}(0.080 \mathrm{~g}, 0.153 \mathrm{mmol}, 1.0$ equiv) in DCM $(7.7 \mathrm{~mL})$ was added $\mathrm{N}$-methylmorpholine $(0.084 \mathrm{~mL}, 0.765 \mathrm{mmol}, 5.0$ equiv), followed by the dropwise addition of trimethylsilyl iodide $(0.065 \mathrm{~mL}, 0.459 \mathrm{mmol}, 3.0$ equiv). The resulting solution was warmed to ambient temperature and stirred for $3 \mathrm{~h}$. The reaction vessel atmosphere was exchanged to $\mathrm{O}_{2}$. To the solution was added a saturated aqueous $\mathrm{NaHCO}_{3}$ solution $(3.85 \mathrm{~mL})$. The biphasic solution was stirred vigorously for $15 \mathrm{~h}$. To the solution was added DCM $(10 \mathrm{~mL})$ and $\mathrm{H}_{2} \mathrm{O}(10 \mathrm{~mL})$. The layers were separated, and the aqueous layer was further extracted with DCM $(2 \times 10 \mathrm{~mL})$. The organic layers were combined, washed with brine $(10 \mathrm{~mL})$, dried over $\mathrm{Na}_{2} \mathrm{SO}_{4}$, filtered, and concentrated under reduced pressure. The residue was purified by chromatography on silica gel (25\% EtOAc in DCM) to afford compound $8 \mathrm{e}(0.024 \mathrm{~g}, 49 \%)$ as a yellow solid.

${ }^{1} \mathrm{H}$ NMR $\left(400 \mathrm{MHz}\right.$, DMSO- $\left.d_{6}\right) \delta=10.32(\mathrm{~s}, 1 \mathrm{H}), 8.64(\mathrm{~s}, 1 \mathrm{H}), 7.85-7.79(\mathrm{~m}, 4 \mathrm{H}), 7.40-7.34(\mathrm{~m}$, $5 \mathrm{H}), 5.67(\mathrm{~s}, 2 \mathrm{H}), 2.10(\mathrm{~s}, 3 \mathrm{H}) \mathrm{ppm}$.

${ }^{13} \mathrm{C}$ NMR $(100 \mathrm{MHz}$, DMSO-d $) \delta=169.9,160.2,147.5,142.8,135.6,128.9,128.4,128.2,123.6$, 119.2, 115.7, 53.6, $24.2 \mathrm{ppm}$.

HRMS (ES $\left.{ }^{+}\right): m / z[M+N a]^{+} \mathrm{C}_{17} \mathrm{H}_{16} \mathrm{~N}_{6} \mathrm{ONa}^{+}$343.1283; Found 343.1297.

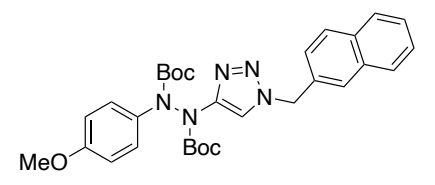

di-tert-butyl yl)hydrazine-1,2-dicarboxylate

To a solution of arylynehydrazide $4 \mathrm{c}(0.075 \mathrm{~g}, 0.207 \mathrm{mmol}, 1.0$ equiv) in THF $(0.41 \mathrm{~mL})$ was added naphthyl azide ${ }^{4}(0.045 \mathrm{~g}, 0.249 \mathrm{mmol}, 1.2$ equiv), triethylamine $(0.035 \mathrm{~mL}, 0.249 \mathrm{mmol}$, 1.2 equiv), and copper( $\mathrm{I})$ iodide $(0.008 \mathrm{~g}, 0.04 \mathrm{mmol}, 0.2$ equiv). The mixture was stirred at ambient temperature for $24 \mathrm{~h}$, diluted with EtOAc $(10 \mathrm{~mL})$, and filtered through celite. The reaction vessel and filter cake were washed with additional EtOAc. The organic solution was concentrated under reduced pressure and purified by chromatography on silica gel (20\% EtOAc in hexanes) to afford di-tert-butyl 1-(4-methoxyphenyl)-2-(1-(naphthalen-2-ylmethyl)-1 $\mathrm{H}$-1,2,3-triazol-4yl)hydrazine-1,2-dicarboxylate $(0.105 \mathrm{~g}, 93 \%)$ as a white semi-solid.

${ }^{1} \mathrm{H}$ NMR $\left(400 \mathrm{MHz}, \mathrm{CDCl}_{3}\right)$ (rotamers observed) $\delta=7.86-7.70(\mathrm{~m}, 5 \mathrm{H}), 7.58-7.51(\mathrm{~m}, 4 \mathrm{H}), 7.37-$ $7.35(\mathrm{~m}, 1 \mathrm{H}), 6.84-6.83(\mathrm{~m}, 2 \mathrm{H}), 5.65-5.56(\mathrm{~m}, 2 \mathrm{H}), 3.78-3.77(\mathrm{~m}, 3 \mathrm{H}), 1.51-1.37(\mathrm{~m}, 18 \mathrm{H}) \mathrm{ppm}$. ${ }^{13} \mathrm{C}$ NMR $\left(100 \mathrm{MHz}, \mathrm{CDCl}_{3}\right)$ (rotamers observed) $\delta 158.2,153.0,152.5,146.6,134.7,134.0$, 133.3, 133.3, 132.0, 131.8, 129.2, 128.1, 127.9, 127.7, 127.6, 126.9, 126.8, 125.8, 125.5, 114.0, $113.9,113.6,113.5,83.4,83.1,82.2,81.8,55.5,55.0,28.3,28.2$ ppm.

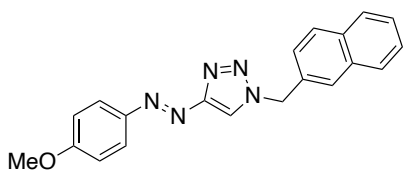


(E)-4-((4-methoxyphenyl)diazenyl)-1-(naphthalen-2-ylmethyl)-1H-1,2,3-triazole (9)

To a $0{ }^{\circ} \mathrm{C}$ cooled solution of Di-tert-butyl 1-(4-methoxyphenyl)-2-(1-(naphthalen-2-ylmethyl)-1 $\mathrm{H}$ 1,2,3-triazol-4-yl)hydrazine-1,2-dicarboxylate $(0.094 \mathrm{~g}, 0.17 \mathrm{mmol}, 1.0$ equiv) in DCM (8.6 $\mathrm{mL})$ was added $\mathrm{N}$-methylmorpholine $(0.095 \mathrm{~mL}, 0.86 \mathrm{mmol}, 5.0$ equiv), followed by the dropwise addition of trimethylsilyl iodide $(0.074 \mathrm{~mL}, 0.52 \mathrm{mmol}, 3.0$ equiv). The resulting solution was warmed to ambient temperature and stirred for $4 \mathrm{~h}$. The reaction vessel atmosphere was exchanged to $\mathrm{O}_{2}$. To the solution was added a saturated aqueous $\mathrm{NaHCO}_{3}$ solution $(4.3 \mathrm{~mL})$. The biphasic solution was stirred vigorously for $20 \mathrm{~h}$. To the solution was added DCM $(20 \mathrm{~mL})$ and $\mathrm{H}_{2} \mathrm{O}(20 \mathrm{~mL})$. The layers were separated and the aqueous layer was further extracted with DCM $(2 \times 20 \mathrm{~mL})$. The organic extracts were combined, washed with brine $(20 \mathrm{~mL})$, dried over $\mathrm{Na}_{2} \mathrm{SO}_{4}$, filtered, and concentrated under reduced pressure. The residue was purified by chromatography on silica gel (100\% DCM to 4\% EtOAc in DCM to afford compound $9(0.037 \mathrm{~g}, 63 \%)$ as a yellow solid.

${ }^{1} \mathrm{H}$ NMR $\left(400 \mathrm{MHz}, \mathrm{CDCl}_{3}\right) \delta=7.90-7.80(\mathrm{~m}, 7 \mathrm{H}), 7.53-7.51(\mathrm{~m}, 2 \mathrm{H}), 7.41-7.38(\mathrm{~m}, 1 \mathrm{H}), 6.97$ (d, $J=9.0 \mathrm{~Hz}, 2 \mathrm{H}), 5.73(\mathrm{~s}, 2 \mathrm{H}), 3.86(\mathrm{~s}, 3 \mathrm{H}) \mathrm{ppm}$.

${ }^{13} \mathrm{C}$ NMR $\left(100 \mathrm{MHz}, \mathrm{CDCl}_{3}\right) \delta=162.8,161.2,147.1,133.4,133.4,131.5,129.5,128.1,128.0$, $127.9,127.0,127.0,125.5,125.2,114.5,114.4,55.7,55.2 \mathrm{ppm}$.

HRMS $\left(\mathrm{ES}^{+}\right): \mathrm{m} / \mathrm{z}[\mathrm{M}+\mathrm{Na}]^{+} \mathrm{C}_{20} \mathrm{H}_{17} \mathrm{~N}_{5} \mathrm{ONa}^{+}$366.1331; Found 366.1338.

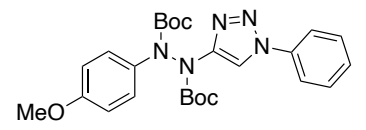

di-tert-butyl dicarboxylate

To a solution of arylynehydrazide $4 \mathrm{c}(0.200 \mathrm{~g}, 0.552 \mathrm{mmol}, 1.0$ equiv) in THF $(1.1 \mathrm{~mL})$ was added phenyl azide ${ }^{5}(0.079 \mathrm{~g}, 0.663 \mathrm{mmol}, 1.2$ equiv), triethylamine $(0.092 \mathrm{~mL}, 0.663 \mathrm{mmol}, 1.2$ equiv), and copper $(\mathrm{I})$ iodide $(0.021 \mathrm{~g}, 0.11 \mathrm{mmol}, 0.2$ equiv). The mixture was stirred at ambient temperature for $24 \mathrm{~h}$, diluted with EtOAc $(20 \mathrm{~mL})$, and filtered through celite. The reaction vessel and filter cake were washed with additional EtOAc. The organic solution was concentrated under reduced pressure and purified by chromatography on silica gel (15\% EtOAc in hexanes) to afford di-tert-butyl 1-(4-methoxyphenyl)-2-(1-phenyl-1H-1,2,3-triazol-4-yl)hydrazine-1,2-dicarboxylate $(0.215 \mathrm{~g}, 81 \%)$ as a white semi-solid.

${ }^{1} \mathrm{H}$ NMR $\left(400 \mathrm{MHz}, \mathrm{CDCl}_{3}\right)$ (rotamers observed) $\delta=8.31-8.21(\mathrm{~m}, 1 \mathrm{H}), 7.80-7.69(\mathrm{~m}, 2 \mathrm{H}), 7.62-$ $7.54(\mathrm{~m}, 2 \mathrm{H}), 7.54-7.45(\mathrm{~m}, 2 \mathrm{H}), 7.45-7.37(\mathrm{~m}, 1 \mathrm{H}), 6.90-6.84(\mathrm{~m}, 2 \mathrm{H}), 3.84-3.75(\mathrm{~m}, 3 \mathrm{H}), 1.63-$ $1.40(\mathrm{~m}, 18 \mathrm{H}) \mathrm{ppm}$.

${ }^{13} \mathrm{C}$ NMR $\left(100 \mathrm{MHz}, \mathrm{CDCl}_{3}\right)$ (rotamers observed) $\delta=158.3,153.1,152.8,152.4,146.9,146.7$, $137.3,134.7,134.0,129.8,128.7,127.8,127.0,120.4,120.2,114.0,113.6,112.2,111.4$, 83.4, 82.4, 82.0, 55.5, 28.4, 28.3, 28.3 ppm.

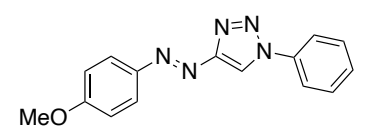

(E)-4-((4-methoxyphenyl)diazenyl)-1-phenyl-1 H-1,2,3-triazole (10)

To a $0{ }^{\circ} \mathrm{C}$ cooled solution of Di-tert-butyl 1-(4-methoxyphenyl)-2-(1-phenyl-1 $\mathrm{H}$-1,2,3-triazol-4yl)hydrazine-1,2-dicarboxylate $(0.165 \mathrm{~g}, 0.343 \mathrm{mmol}, 1.0$ equiv) in DCM (17.2 mL) was added $\mathrm{N}$ methylmorpholine $(0.189 \mathrm{~mL}, 1.715 \mathrm{mmol}, 5.0$ equiv), followed by the dropwise addition of trimethylsilyl iodide $\left(0.146 \mathrm{~mL}, 1.028 \mathrm{mmol}, 3.0\right.$ equiv). The resulting solution was stirred at $40^{\circ} \mathrm{C}$ for $24 \mathrm{~h}$. The reaction vessel was cooled to ambient temperature and the atmosphere was exchanged to $\mathrm{O}_{2}$. To the solution was added a saturated aqueous $\mathrm{NaHCO}_{3}$ solution $(8.6 \mathrm{~mL})$. The 
biphasic solution was stirred vigorously for $24 \mathrm{~h}$. To the solution was added DCM $(20 \mathrm{~mL})$ and $\mathrm{H}_{2} \mathrm{O}(20 \mathrm{~mL})$. The layers were separated and the aqueous layer was further extracted with DCM $(2 \times 20 \mathrm{~mL})$. The organic extracts were combined, washed with brine $(20 \mathrm{~mL})$, dried over $\mathrm{Na}_{2} \mathrm{SO}_{4}$, filtered, and concentrated under reduced pressure. The residue was purified by chromatography on silica gel $(20 \%$ hexanes in DCM) to afford compound $10(0.071 \mathrm{~g}, 74 \%)$ as a yellow solid.

${ }^{1} \mathrm{H}$ NMR $\left(400 \mathrm{MHz}, \mathrm{CDCl}_{3}\right) \delta=8.33(\mathrm{~s}, 1 \mathrm{H}), 7.97(\mathrm{~d}, J=9.0 \mathrm{~Hz}, 2 \mathrm{H}), 7.83-7.81(\mathrm{~m}, 2 \mathrm{H}), 7.55(\mathrm{t}$, $J=7.3 \mathrm{~Hz}, 2 \mathrm{H}), 7.47(\mathrm{t}, J=7.4 \mathrm{~Hz}, 1 \mathrm{H}), 7.02(\mathrm{~d}, J=9.1 \mathrm{~Hz}, 2 \mathrm{H}), 3.89(\mathrm{~s}, 3 \mathrm{H}) \mathrm{ppm}$.

${ }^{13} \mathrm{C}$ NMR $\left(100 \mathrm{MHz}, \mathrm{CDCl}_{3}\right) \delta=162.9,161.2,147.1,137.0,130.0,129.4,125.3,120.7,114.5$, 112.2, $55.8 \mathrm{ppm}$.

HRMS $\left(\mathrm{ES}^{+}\right): \mathrm{m} / \mathrm{z}[\mathrm{M}+\mathrm{Na}]^{+}$Calcd for $\mathrm{C}_{15} \mathrm{H}_{13} \mathrm{~N}_{5} \mathrm{ONa}^{+}$302.1018; Found 302.1023.

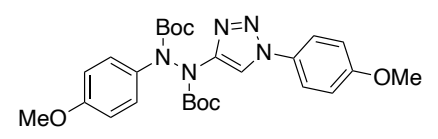

di-tert-butyl 1-(4-methoxyphenyl)-2-(1-(4-methoxyphenyl)-1H-1,2,3-triazol-4-yl)hydrazine1,2-dicarboxylate

To a solution of arylynehydrazide $4 \mathrm{c}(0.062 \mathrm{~g}, 0.171 \mathrm{mmol}, 1.0$ equiv) in THF $(0.4 \mathrm{~mL})$ was added 1-azido-4-methoxybenzene ${ }^{6}(0.031 \mathrm{~g}, 0.205 \mathrm{mmol}, 1.2$ equiv), triethylamine $(0.029 \mathrm{~mL}, 0.205$ mmol, 1.2 equiv), and copper(I) iodide $(0.007 \mathrm{~g}, 0.034 \mathrm{mmol}, 0.2$ equiv). The mixture was stirred at ambient temperature for $26 \mathrm{~h}$, diluted with $\mathrm{H}_{2} \mathrm{O}(20 \mathrm{~mL})$, and extracted with EtOAc $(3 \times 30 \mathrm{~mL})$. The organic extracts were combined, washed with brine $(30 \mathrm{~mL})$, dried over $\mathrm{Na}_{2} \mathrm{SO}_{4}$, filtered, and concentrated under reduced pressure. The residue was purified by chromatography on silica gel (25\% EtOAc in hexanes) to afford di-tert-butyl 1-(4-methoxyphenyl)-2-(1-(4-methoxyphenyl)-1H1,2,3-triazol-4-yl)hydrazine-1,2-dicarboxylate $(0.096 \mathrm{~g},>99 \%)$ as a white semi-solid.

${ }^{1} \mathrm{H}$ NMR $\left(600 \mathrm{MHz}, \mathrm{CDCl}_{3}\right)$ (rotamers observed) $\delta=8.20-8.10(\mathrm{~m}, 1 \mathrm{H}), 7.65-7.52(\mathrm{~m}, 4 \mathrm{H}), 7.03-$ $6.96(\mathrm{~m}, 2 \mathrm{H}), 6.88-6.83(\mathrm{~m}, 2 \mathrm{H}), 3.84(\mathrm{~s}, 3 \mathrm{H}), 3.80-3.75(\mathrm{~m}, 3 \mathrm{H}), 1.60-1.42(\mathrm{~m}, 18 \mathrm{H}) \mathrm{ppm}$.

${ }^{13} \mathrm{C}$ NMR $\left(150 \mathrm{MHz} \mathrm{CDCl}_{3}\right.$ ) (rotamers observed) $\delta=159.8,158.2,153.1,152.8,152.4,146.7$, $146.5,134.6,133.9,130.9,130.8,127.8,127.0,122.0,121.8,114.8,114.8,113.9,113.5,112.3$, $111.6,83.3,83.3,82.3,81.9,55.7,55.5,55.5,28.4,28.3,28.3,28.3$ ppm.

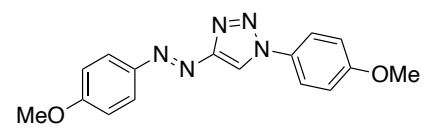

(E)-1-(4-methoxyphenyl)-4-((4-methoxyphenyl)diazenyl)-1H-1,2,3-triazole (11)

To a $0^{\circ} \mathrm{C}$ cooled solution of Di-tert-butyl 1-(4-methoxyphenyl)-2-(1-(4-methoxyphenyl)-1 H-1,2,3triazol-4-yl)hydrazine-1,2-dicarboxylate $(0.151 \mathrm{~g}, 0.295 \mathrm{mmol}, 1.0$ equiv) in DCM $(14.8 \mathrm{~mL})$ was added $\mathrm{N}$-methylmorpholine $(0.162 \mathrm{~mL}, 1.477 \mathrm{mmol}, 5.0$ equiv), followed by the dropwise addition of trimethylsilyl iodide $(0.126 \mathrm{~mL}, 0.886 \mathrm{mmol}, 3.0$ equiv). The resulting solution was stirred at $40^{\circ} \mathrm{C}$ for $24 \mathrm{~h}$. The reaction vessel was cooled to ambient temperature and the atmosphere was exchanged to $\mathrm{O}_{2}$. To the solution was added a saturated aqueous $\mathrm{NaHCO}_{3}$ solution $(7.4 \mathrm{~mL})$. The biphasic solution was stirred vigorously for $24 \mathrm{~h}$. To the solution was added DCM $(10 \mathrm{~mL})$ and $\mathrm{H}_{2} \mathrm{O}(10 \mathrm{~mL})$. The layers were separated, and the aqueous layer was further extracted with DCM $(2 \times 10 \mathrm{~mL})$. The organic extracts were combined, washed with brine $(20 \mathrm{~mL})$, dried over $\mathrm{Na}_{2} \mathrm{SO}_{4}$, filtered, and concentrated under reduced pressure. The residue was purified by chromatography on silica gel (2\% EtOAc in DCM to 3\% EtOAc in DCM) to afford compound $11(0.075 \mathrm{~g}, 82 \%)$ as a yellow solid.

${ }^{1} \mathrm{H}$ NMR $\left(600 \mathrm{MHz}, \mathrm{CDCl}_{3}\right) \delta=8.25(\mathrm{~s}, 1 \mathrm{H}), 7.97(\mathrm{~d}, J=5.8 \mathrm{~Hz}, 2 \mathrm{H}), 7.71(\mathrm{~d}, J=5.9 \mathrm{~Hz}, 2 \mathrm{H})$, 7.06-6.99 (m, 4H), $3.38(\mathrm{~s}, 3 \mathrm{H}), 3.88(\mathrm{~s}, 3 \mathrm{H}) \mathrm{ppm}$. 
${ }^{13} \mathrm{C}$ NMR $\left(150 \mathrm{MHz}, \mathrm{CDCl}_{3}\right) \delta=162.9,161.1,160.3,147.1,130.3,125.3,122.3,115.0,114.5$, 112.3, 55.8, $55.8 \mathrm{ppm}$.

HRMS $\left(\mathrm{ES}^{+}\right): \mathrm{m} / \mathrm{z}[\mathrm{M}+\mathrm{Na}]^{+}$Calcd for $\mathrm{C}_{16} \mathrm{H}_{15} \mathrm{~N}_{5} \mathrm{O}_{2} \mathrm{Na}^{+}$332.1123; Found 332.1136.

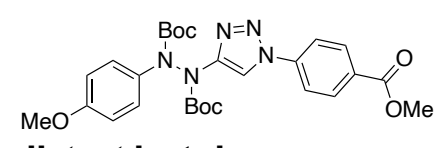

di-tert-butyl methoxyphenyl)hydrazine-1,2-dicarboxylate

To a solution of arylynehydrazide $4 \mathrm{c}(0.100 \mathrm{~g}, 0.276 \mathrm{mmol}, 1.0$ equiv) in THF $(0.55 \mathrm{~mL})$ was added methyl 4-azidobenzoate $(0.059 \mathrm{~g}, 0.33 \mathrm{mmol}, 1.2$ equiv), triethylamine $(0.046 \mathrm{~mL}, 0.33$ $\mathrm{mmol}, 1.2$ equiv), and copper $(\mathrm{l})$ iodide $(0.011 \mathrm{~g}, 0.06 \mathrm{mmol}, 0.2$ equiv). The mixture was stirred at ambient temperature for $26 \mathrm{~h}$, diluted with EtOAc $(10 \mathrm{~mL})$, and filtered through celite. The reaction vessel and filter cake were washed with additional EtOAc. The organic solution was concentrated under reduced pressure and purified by chromatography on silica gel $(20 \%$ EtOAc in hexanes) to afford di-tert-butyl 1-(1-(4-(methoxycarbonyl)phenyl)-1H-1,2,3-triazol-4-yl)-2-(4methoxyphenyl)hydrazine-1,2-dicarboxylate $(0.144 \mathrm{~g}, 97 \%)$ as a white semi-solid.

${ }^{1} \mathrm{H}$ NMR $\left(400 \mathrm{MHz}, \mathrm{CDCl}_{3}\right)$ (rotamers observed) $\delta=8.38-8.28(\mathrm{~m}, 1 \mathrm{H}), 8.20-8.12(\mathrm{~m}, 2 \mathrm{H}) 7.89$ $7.78(\mathrm{~m}, 2 \mathrm{H}), 7.59-7.52(\mathrm{~m}, 2 \mathrm{H}), 6.85(\mathrm{~d}, J=8.8 \mathrm{~Hz}, 2 \mathrm{H}), 3.92(\mathrm{~s}, 3 \mathrm{H}), 3.79-3.73(\mathrm{~m}, 3 \mathrm{H}), 1.61-$ $1.39(\mathrm{~m}, 18 \mathrm{H}) \mathrm{ppm}$.

${ }^{13} \mathrm{C}$ NMR $\left(100 \mathrm{MHz}, \mathrm{CDCl}_{3}\right.$ ) (rotamers observed) $\delta=166.0,158.3,153.0,152.7,152.3,147.2$, 147.0, 140.3, 140.2, 134.5, 133.8, 131.6, 131.3, 130.1, 127.7, 127.0, 119.6, 119.5, 113.9, 113.8, 113.6, 111.7, 111.0, 84.1, 83.5, 83.5, 82.4, 82.0, 55.5, 55.4, 52.5, 28.3, 28.2 ppm.

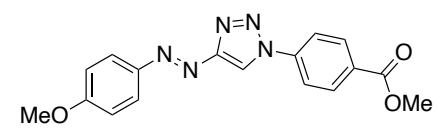

methyl (E)-4-(4-((4-methoxyphenyl)diazenyl)-1H-1,2,3-triazol-1-yl)benzoate (12)

To a $0^{\circ} \mathrm{C}$ cooled solution of Di-tert-butyl 1-(1-(4-(methoxycarbonyl)phenyl)-1H-1,2,3-triazol-4-yl)2-(4-methoxyphenyl)hydrazine-1,2-dicarboxylate $(0.140 \mathrm{~g}, 0.269 \mathrm{mmol}, 1.0$ equiv) in DCM (13.5 $\mathrm{mL}$ ) was added $\mathrm{N}$-methylmorpholine $(0.148 \mathrm{~mL}, 1.345 \mathrm{mmol}, 5.0$ equiv), followed by the dropwise addition of trimethylsilyl iodide $(0.111 \mathrm{~mL}, 0.808 \mathrm{mmol}, 3.0$ equiv). The resulting solution was warmed to ambient temperature and stirred for $17 \mathrm{~h}$. The reaction vessel atmosphere was exchanged to $\mathrm{O}_{2}$. To the solution was added a saturated aqueous $\mathrm{NaHCO}_{3}$ solution $(6.75 \mathrm{~mL})$. The biphasic solution was stirred vigorously for $12 \mathrm{~h}$. To the solution was added DCM $(40 \mathrm{~mL})$ and $\mathrm{H}_{2} \mathrm{O}(30 \mathrm{~mL})$. The layers were separated, and the aqueous layer was further extracted with DCM $(2 \times 40 \mathrm{~mL})$. The organic extracts were combined, washed with brine $(50 \mathrm{~mL})$, dried over $\mathrm{Na}_{2} \mathrm{SO}_{4}$, filtered, and concentrated under reduced pressure. The residue was purified by chromatography on silica gel (DCM to $10 \%$ EtOAc in DCM) to afford compound $12(0.027 \mathrm{~g}, 30 \%)$ as a yellow solid.

${ }^{1} \mathrm{H}$ NMR $\left(400 \mathrm{MHz}, \mathrm{CDCl}_{3}\right) \delta=8.39(\mathrm{~s}, 1 \mathrm{H}), 8.24(\mathrm{~d}, J=8.7 \mathrm{~Hz}, 2 \mathrm{H}), 7.99-7.91(\mathrm{~m}, 4 \mathrm{H}), 7.02(\mathrm{~d}$, $J=9.0 \mathrm{~Hz}, 2 \mathrm{H}), 3.97(\mathrm{~s}, 3 \mathrm{H}), 3.90(\mathrm{~s}, 3 \mathrm{H}) \mathrm{ppm}$.

${ }^{13} \mathrm{C}$ NMR $\left(100 \mathrm{MHz}, \mathrm{CDCl}_{3}\right) \delta=165.9,163.1,161.4,147.1,140.0,131.6,130.8,125.4,120.1$, 114.5, 111.9, 55.8, $52.7 \mathrm{ppm}$.

HRMS (ES $\left.{ }^{+}\right): m / z[M+N a]^{+}$Calcd for $\mathrm{C}_{17} \mathrm{H}_{15} \mathrm{~N}_{5} \mathrm{O}_{3} \mathrm{Na}^{+} 360.1073$; Found 360.1087. 


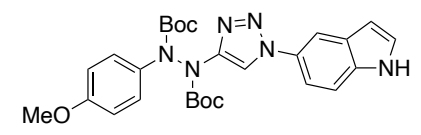

di-tert-butyl 1-(1-(1H-indol-5-yl)-1H-1,2,3-triazol-4-yl)-2-(4-methoxyphenyl)hydrazine-1,2dicarboxylate

To a solution of arylynehydrazide $4 \mathrm{c}(0.191 \mathrm{~g}, 0.527 \mathrm{mmol}, 1.0$ equiv) in THF $(1.05 \mathrm{~mL})$ was added 5 -azido- $1 \mathrm{H}$-indole ${ }^{7}(0.100 \mathrm{~g}, 0.632 \mathrm{mmol}, 1.2$ equiv), triethylamine $(0.09 \mathrm{~mL}, 0.632 \mathrm{mmol}$, 1.2 equiv), and copper( $(\mathrm{l})$ iodide $(0.020 \mathrm{~g}, 0.105 \mathrm{mmol}, 0.2$ equiv). The mixture was stirred at ambient temperature for $24 \mathrm{~h}$, diluted with $\mathrm{H}_{2} \mathrm{O}(40 \mathrm{~mL})$, and extracted with EtOAc $(3 \times 60 \mathrm{~mL})$. The organic extracts were combined, washed with brine $(60 \mathrm{~mL})$, dried over $\mathrm{Na}_{2} \mathrm{SO}_{4}$, filtered, and concentrated under reduced pressure. The residue was purified by chromatography on silica gel (33\% EtOAc in hexanes) to afford di-tert-butyl 1-(1-(1H-indol-5-yl)-1H-1,2,3-triazol-4-yl)-2-(4methoxyphenyl)hydrazine-1,2-dicarboxylate $(0.263 \mathrm{~g}, 96 \%)$ as an off-white solid.

${ }^{1} \mathrm{H}$ NMR $\left(600 \mathrm{MHz}, \mathrm{CDCl}_{3}\right)$ (rotamers observed) $\delta=9.77-9.61(\mathrm{~m}, 1 \mathrm{H}), 8.28-8.20(\mathrm{~m}, 1 \mathrm{H}), 7.94-$ $7.74(\mathrm{~m}, 1 \mathrm{H}), 7.69-7.58(\mathrm{~m}, 2 \mathrm{H}), 7.54-7.32(\mathrm{~m}, 2 \mathrm{H}), 7.24-7.15(\mathrm{~m}, 1 \mathrm{H}), 6.91-6.84(\mathrm{~m}, 2 \mathrm{H}), 6.58-$ $6.50(\mathrm{~m}, 1 \mathrm{H}), 3.82-3.72(\mathrm{~m}, 3 \mathrm{H}), 1.66-1.42(\mathrm{~m}, 18 \mathrm{H}) \mathrm{ppm}$.

${ }^{13} \mathrm{C} \mathrm{NMR}\left(150 \mathrm{MHz}, \mathrm{CDCl}_{3}\right)$ (rotamers observed) $\delta=158.2,158.2,153.3,152.7,152.3,146.2$, 135.8, 135.7, 134.4, 133.8, 130.1, 130.0, 127.9, 127.8, 127.4, 127.2, 127.0, 126.9, 126.8, 114.8, $113.9,113.7,113.6,113.1,112.7,112.6,112.4,112.2,102.5,83.3,82.6,82.1,55.4,28.2,28.2$, $28.0 \mathrm{ppm}$.

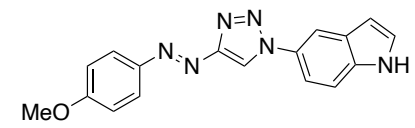

(E)-5-(4-((4-methoxyphenyl)diazenyl)-1H-1,2,3-triazol-1-yl)-1H-indole (13)

To a $0^{\circ} \mathrm{C}$ cooled solution of Di-tert-butyl 1-(1-(1H-indol-5-yl)-1H-1,2,3-triazol-4-yl)-2-(4methoxyphenyl)hydrazine-1,2-dicarboxylate $(0.095 \mathrm{~g}, 0.182 \mathrm{mmol}, 1.0$ equiv) in DCM $(9.0 \mathrm{~mL})$ was added $\mathrm{N}$-methylmorpholine $(0.10 \mathrm{~mL}, 0.908 \mathrm{mmol}, 5.0$ equiv), followed by the dropwise addition of trimethylsilyl iodide $(0.078 \mathrm{~mL}, 0.545 \mathrm{mmol}, 3.0$ equiv). The resulting solution was stirred at $40^{\circ} \mathrm{C}$ for $24 \mathrm{~h}$. The reaction vessel was cooled to ambient temperature and the atmosphere was exchanged to $\mathrm{O}_{2}$. To the solution was added a saturated aqueous $\mathrm{NaHCO}_{3}$ solution $(4.5 \mathrm{~mL})$. The biphasic solution was stirred vigorously for $24 \mathrm{~h}$. To the solution was added DCM $(10 \mathrm{~mL})$ and $\mathrm{H}_{2} \mathrm{O}(10 \mathrm{~mL})$. The layers were separated, and the aqueous layer was further extracted with DCM $(2 \times 10 \mathrm{~mL})$. The organic extracts were combined, washed with brine $(20 \mathrm{~mL})$, dried over $\mathrm{Na}_{2} \mathrm{SO}_{4}$, filtered, and concentrated under reduced pressure. The residue was purified by chromatography on silica gel (3\% EtOAc in DCM) to afford compound $13(0.040 \mathrm{~g}, 70 \%)$ as a yellow solid.

${ }^{1} \mathrm{H}$ NMR $\left(600 \mathrm{MHz}\right.$, DMSO- $\left.d_{6}\right) \delta=11.48(\mathrm{~s}, 1 \mathrm{H}), 9.14(\mathrm{~s}, 1 \mathrm{H}), 8.17-8.16(\mathrm{~m}, 1 \mathrm{H}), 7.02(\mathrm{~d}, J=5.7$ $\mathrm{Hz}, 2 \mathrm{H}), 7.71(\mathrm{~d}, J=5.8 \mathrm{~Hz}, 1 \mathrm{H}), 7.61(\mathrm{~d}, J=5.8 \mathrm{~Hz}, 1 \mathrm{H}), 7.53-7.51(\mathrm{~m}, 1 \mathrm{H}), 7.16(\mathrm{~d}, J=5.8 \mathrm{~Hz}$, $2 \mathrm{H}), 6.61-6.59(\mathrm{~m}, 1 \mathrm{H}), 3.88(\mathrm{~s}, 3 \mathrm{H}) \mathrm{ppm}$.

${ }^{13} \mathrm{C}$ NMR $\left(150 \mathrm{MHz}\right.$, DMSO- $\left.d_{6}\right) \delta=162.4,160.4,146.4,135.8,129.2,127.9,127.7,124.6,114.8$, $114.4,114.0,112.4,112.4,102.1,55.8 \mathrm{ppm}$.

HRMS $\left(\mathrm{ES}^{+}\right): \mathrm{m} / \mathrm{z}[\mathrm{M}+\mathrm{Na}]^{+}$Calcd for $\mathrm{C}_{17} \mathrm{H}_{14} \mathrm{~N}_{6} \mathrm{ONa}^{+} 341.1127$; Found 341.1137.

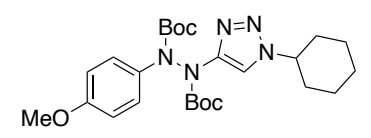

di-tert-butyl

1-(1-cyclohexyl-1H-1,2,3-triazol-4-yl)-2-(4-methoxyphenyl)hydrazine-1,2dicarboxylate 
To a solution of arylynehydrazide $4 \mathrm{c}(0.165 \mathrm{~g}, 0.456 \mathrm{mmol}, 1.0$ equiv) in THF $(0.91 \mathrm{~mL})$ was added cyclohexyl azide ${ }^{8}(0.068 \mathrm{~g}, 0.547 \mathrm{mmol}, 1.2$ equiv), triethylamine $(0.076 \mathrm{~mL}, 0.547 \mathrm{mmol}$, 1.2 equiv), and copper(I) iodide $(0.017 \mathrm{~g}, 0.09 \mathrm{mmol}, 0.2$ equiv). The mixture was stirred at ambient temperature for $24 \mathrm{~h}$, diluted with EtOAc $(10 \mathrm{~mL})$, and filtered through celite. The reaction vessel and filter cake were washed with additional EtOAc. The organic solution was concentrated under reduced pressure and purified by chromatography on silica gel (10\% EtOAc in hexanes to $20 \%$ EtOAc in hexanes) to afford di-tert-butyl 1-(1-cyclohexyl-1H-1,2,3-triazol-4-yl)-2-(4methoxyphenyl)hydrazine-1,2-dicarboxylate $(0.164 \mathrm{~g}, 74 \%)$ as an off white semi-solid.

${ }^{1} \mathrm{H}$ NMR $\left(400 \mathrm{MHz}, \mathrm{CDCl}_{3}\right)$ (rotamers observed) $\delta=7.82-7.72(\mathrm{~m}, 1 \mathrm{H}), 7.60-7.50(\mathrm{~m}, 2 \mathrm{H}), 6.87-$ $6.81(\mathrm{~m}, 2 \mathrm{H}), 4.43-4.29(\mathrm{~m}, 1 \mathrm{H}), 3.80-3.74(\mathrm{~m}, 3 \mathrm{H}), 2.25-2.15(\mathrm{~m}, 2 \mathrm{H}), 1.94-1.85(\mathrm{~m}, 2 \mathrm{H}), 1.79$ $1.66(\mathrm{~m}, 3 \mathrm{H}), 1.58-1.36(\mathrm{~m}, 2 \mathrm{H}), 1.30-1.19(\mathrm{~m}, 1 \mathrm{H}) \mathrm{ppm}$.

${ }^{13} \mathrm{C}$ NMR $\left(100 \mathrm{MHz}, \mathrm{CDCl}_{3}\right)$ (rotamers observed) $\delta=158.2,153.1,152.9,152.6,145.8,134.8$, $134.1,127.8,127.0,113.9,113.5,112.0,111.4$, 83.0, 82.2, 81.7, 60.6, 55.5, 33.5, 28.3, 28.3, $25.3 \mathrm{ppm}$.

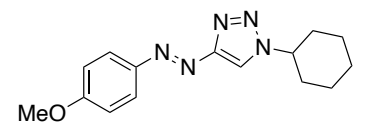

(E)-1-cyclohexyl-4-((4-methoxyphenyl)diazenyl)-1H-1,2,3-triazole (14)

To a $0 \quad{ }^{\circ} \mathrm{C}$ cooled solution of Di-tert-butyl 1-(1-cyclohexyl-1H-1,2,3-triazol-4-yl)-2-(4methoxyphenyl)hydrazine-1,2-dicarboxylate $(0.100 \mathrm{~g}, 0.205 \mathrm{mmol}, 1.0$ equiv) in DCM (10.25 mL) was added $\mathrm{N}$-methylmorpholine $(0.112 \mathrm{~mL}, 1.026 \mathrm{mmol}, 5.0$ equiv), followed by the dropwise addition of trimethylsilyl iodide $(0.09 \mathrm{~mL}, 0.616 \mathrm{mmol}, 3.0$ equiv). The resulting solution was warmed to ambient temperature and stirred for $3 \mathrm{~h}$. The reaction vessel atmosphere was exchanged to $\mathrm{O}_{2}$. To the solution was added a saturated aqueous $\mathrm{NaHCO}_{3}$ solution $(5 \mathrm{~mL})$. The biphasic solution was stirred vigorously for $16 \mathrm{~h}$. To the solution was added DCM (10 mL) and $\mathrm{H}_{2} \mathrm{O}(10 \mathrm{~mL})$. The layers were separated, and the aqueous layer was further extracted with DCM $(2 \times 10 \mathrm{~mL})$. The organic extracts were combined, washed with brine $(10 \mathrm{~mL})$, dried over $\mathrm{Na}_{2} \mathrm{SO}_{4}$, filtered, and concentrated under reduced pressure. The residue was purified by chromatography on silica gel (DCM to 5\% EtOAc in DCM) to afford compound $14(0.050 \mathrm{~g}, 85 \%)$ as a burnt-orange solid.

${ }^{1} \mathrm{H}$ NMR $\left(400 \mathrm{MHz}, \mathrm{CDCl}_{3}\right) \delta=7.94-7.91(\mathrm{~m}, 3 \mathrm{H}), 6.99(\mathrm{~d}, \mathrm{~J}=9.0 \mathrm{~Hz}, 2 \mathrm{H}), 4.49(\mathrm{tt}, \mathrm{J}=11.8 \mathrm{~Hz}$, $J=3.9 \mathrm{~Hz}, 1 \mathrm{H}), 3.87(\mathrm{~s}, 3 \mathrm{H}), 2.28-2.26(\mathrm{~m}, 2 \mathrm{H}), 1.96-1.92(\mathrm{~m}, 2 \mathrm{H}), 1.83-1.74(\mathrm{~m}, 3 \mathrm{H}), 1.53-$ $1.43(\mathrm{~m}, 2 \mathrm{H}), 1.33-1.24(\mathrm{~m}, 1 \mathrm{H}) \mathrm{ppm}$.

${ }^{13} \mathrm{C}$ NMR $\left(100 \mathrm{MHz}, \mathrm{CDCl}_{3}\right) \delta=162.6,160.5,147.1,125.1,114.4,112.3,60.8,55.7,33.6,25.2$, $25.2 \mathrm{ppm}$.

HRMS $\left(\mathrm{ES}^{+}\right): m / z[\mathrm{M}+\mathrm{Na}]^{+}$Calcd for $\mathrm{C}_{15} \mathrm{H}_{19} \mathrm{~N}_{5} \mathrm{Ona}^{+}$308.1487; Found 308.1496.

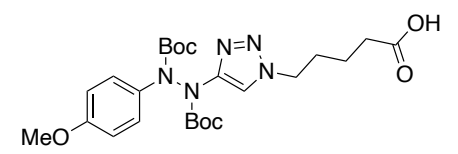

\section{5-(4-(1,2-bis(tert-butoxycarbonyl)-2-(4-methoxyphenyl)hydrazineyl)-1H-1,2,3-triazol-1-} yl)pentanoic acid

To a solution of arylynehydrazide $4 \mathrm{c}(0.095 \mathrm{~g}, 0.262 \mathrm{mmol}, 1.0$ equiv $)$ in THF $(0.5 \mathrm{~mL})$ was added 5-azidopentanoic acid $^{9}(0.045 \mathrm{~g}, 0.315 \mathrm{mmol}, 1.2$ equiv $)$, triethylamine $(0.09 \mathrm{~mL}, 0.315 \mathrm{mmol}, 1.2$ equiv), and copper(I) iodide $(0.010 \mathrm{~g}, 0.05 \mathrm{mmol}, 0.2$ equiv). The mixture was stirred at ambient temperature for $18 \mathrm{~h}$, diluted with EtOAc $(10 \mathrm{~mL})$, and filtered through celite. The reaction vessel and filter cake were washed with additional EtOAc. The organic solution was concentrated under 
reduced pressure and purified by chromatography on silica gel $(50 \%$ EtOAc in hexanes $+0.5 \%$ $\mathrm{AcOH})$ to afford 5-(4-(1,2-bis(tert-butoxycarbonyl)-2-(4-methoxyphenyl)hydrazineyl)-1 H-1,2,3triazol-1-yl)pentanoic acid $(0.099 \mathrm{~g}, 75 \%)$ as a pale yellow semi-solid.

${ }^{1} \mathrm{H}$ NMR $\left(400 \mathrm{MHz}, \mathrm{CDCl}_{3}\right)$ (rotamers observed) $\delta=7.81-7.75(\mathrm{~m}, 1 \mathrm{H}), 7.54-7.50(\mathrm{~m}, 2 \mathrm{H}), 6.83$ $(\mathrm{d}, J=8.3 \mathrm{~Hz}, 2 \mathrm{H}), 4.32-4.29(\mathrm{~m}, 2 \mathrm{H}), 3.77-3.76(\mathrm{~m}, 3 \mathrm{H}), 2.37(\mathrm{t}, J=7.0 \mathrm{~Hz}, 2 \mathrm{H}), 1.97-1.94(\mathrm{~m}$, $2 \mathrm{H}), 1.67-1.65(\mathrm{~m}, 2 \mathrm{H}), 1.54-1.41(\mathrm{~m}, 18 \mathrm{H}) \mathrm{ppm}$.

${ }^{13} \mathrm{C}$ NMR $\left(100 \mathrm{MHz}, \mathrm{CDCl}_{3}\right.$ ) (rotamers observed) $\delta=178.5,158.2,153.1,152.5,146.2,134.6$, 134.0, 127.6, 126.8, 114.3, 113.9, 113.6, 83.3, 82.4, 81.9, 55.5, 50.4, 33.4, 29.4, 28.3, 28.3, 21.7 ppm.

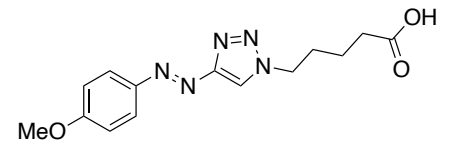

(E)-5-(4-((4-methoxyphenyl)diazenyl)-1H-1,2,3-triazol-1-yl)pentanoic acid (15)

To a $0^{\circ} \mathrm{C}$ cooled solution of 5-(4-(1,2-bis(tert-butoxycarbonyl)-2-(4-methoxyphenyl)hydrazineyl)$1 \mathrm{H}$-1,2,3-triazol-1-yl)pentanoic acid $(0.084 \mathrm{~g}, 0.166 \mathrm{mmol}, 1.0$ equiv) in DCM $(8.3 \mathrm{~mL})$ was added $\mathrm{N}$-methylmorpholine $(0.091 \mathrm{~mL}, 0.831 \mathrm{mmol}, 5.0$ equiv), followed by the dropwise addition of trimethylsilyl iodide $(0.071 \mathrm{~mL}, 0.5 \mathrm{mmol}, 3.0$ equiv). The resulting solution was allowed to warm to ambient temperature and stir for $4 \mathrm{~h}$. The reaction vessel atmosphere was exchanged to $\mathrm{O}_{2}$. To the solution was added a saturated aqueous $\mathrm{NaHCO}_{3}$ solution $(4.15 \mathrm{~mL})$. The biphasic solution was stirred vigorously for $14 \mathrm{~h}$. To the solution was added DCM $(20 \mathrm{~mL})$ and $1 \mathrm{M} \mathrm{HCl}(40 \mathrm{~mL})$. The layers were separated, and the aqueous layer was further extracted with DCM $(2 \times 20 \mathrm{~mL})$. The organic extracts were combined, washed with brine $(20 \mathrm{~mL})$, dried over $\mathrm{Na}_{2} \mathrm{SO}_{4}$, filtered, and concentrated under reduced pressure. The residue was purified by chromatography on silica gel (3\% MeOH in DCM) to afford compound $15(0.026 \mathrm{~g}, 52 \%)$ as a yellow solid.

${ }^{1} \mathrm{H}$ NMR $\left(400 \mathrm{MHz}, \mathrm{DMSO}-\mathrm{d}_{6}\right) \delta=8.56(\mathrm{~s}, 1 \mathrm{H}), 7.86(\mathrm{~d}, J=6.0 \mathrm{~Hz}, 2 \mathrm{H}), 7.13(\mathrm{~d}, J=6.0 \mathrm{~Hz}, 2 \mathrm{H})$, $4.44(\mathrm{t}, J=4.6 \mathrm{~Hz}, 2 \mathrm{H}$ ), $3.86(\mathrm{~s}, 3 \mathrm{H}), 2.26(\mathrm{t}, J=4.9 \mathrm{~Hz}, 2 \mathrm{H}), 1.89$ (quint, $J=5.2 \mathrm{~Hz}, 2 \mathrm{H}$ ), 1.47 (quint, $J=5.0 \mathrm{~Hz}, 2 \mathrm{H}$ ) ppm.

${ }^{13} \mathrm{C}$ NMR $\left(100 \mathrm{MHz}\right.$, DMSO- $\left.d_{6}\right) \delta=174.3,162.3,160.0,146.3,124.5,115.6,114.8,55.7,49.9$, 33.0, 29.0, $21.4 \mathrm{ppm}$.

HRMS $\left(\mathrm{ES}^{+}\right): \mathrm{m} / \mathrm{z}[\mathrm{M}+\mathrm{Na}]^{+}$Calcd for $\mathrm{C}_{14} \mathrm{H}_{17} \mathrm{~N}_{5} \mathrm{O}_{3} \mathrm{Na}^{+}$326.1229; Found 326.1241.

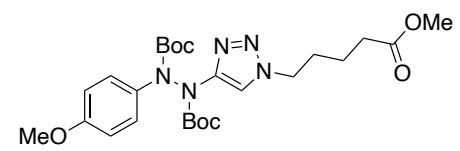

di-tert-butyl

1-(1-(5-methoxy-5-oxopentyl)-1H-1,2,3-triazol-4-yl)-2-(4methoxyphenyl)hydrazine-1,2-dicarboxylate

To a solution of arylynehydrazide $4 \mathrm{c}(0.120 \mathrm{~g}, 0.33 \mathrm{mmol}, 1.0$ equiv $)$ in THF $(0.7 \mathrm{~mL})$ was added methyl 5-azidopentanoate ${ }^{9}(0.062 \mathrm{~g}, 0.398 \mathrm{mmol}, 1.2$ equiv), triethylamine $(0.055 \mathrm{~mL}, 0.398$ mmol, 1.2 equiv), and copper $(\mathrm{I})$ iodide $(0.013 \mathrm{~g}, 0.07 \mathrm{mmol}, 0.2$ equiv). The mixture was stirred at ambient temperature for $24 \mathrm{~h}$, diluted with EtOAc $(20 \mathrm{~mL})$, and filtered through celite. The reaction vessel and filter cake were washed with additional EtOAc. The organic solution was concentrated under reduced pressure and purified by chromatography on silica gel (30\% EtOAc in hexanes) to afford di-tert-butyl 1-(1-(5-methoxy-5-oxopentyl)-1H-1,2,3-triazol-4-yl)-2-(4methoxyphenyl)hydrazine-1,2-dicarboxylate $(0.143 \mathrm{~g}, 82 \%)$ as a white semi-solid.

${ }^{1} \mathrm{H}$ NMR $\left(400 \mathrm{MHz}, \mathrm{CDCl}_{3}\right)$ (rotamers observed) $\delta=7.80-7.74(\mathrm{~m}, 1 \mathrm{H}), 7.56-7.51(\mathrm{~m}, 2 \mathrm{H}), 6.85-$ $6.83(\mathrm{~m}, 2 \mathrm{H}), 4.31-4.28(\mathrm{~m}, 2 \mathrm{H}), 3.79-3.77(\mathrm{~m}, 3 \mathrm{H}), 3.66(\mathrm{~s}, 3 \mathrm{H}), 2.35(\mathrm{t}, J=7.3 \mathrm{~Hz}, 2 \mathrm{H}), 1.97-$ $1.93(\mathrm{~m}, 2 \mathrm{H}), 1.70-1.63(\mathrm{~m}, 2 \mathrm{H}), 1.55-1.41(\mathrm{~m}, 18 \mathrm{H}) \mathrm{ppm}$. 
${ }^{13} \mathrm{C} \mathrm{NMR}\left(100 \mathrm{MHz}, \mathrm{CDCl}_{3}\right)$ (rotamers observed) $\delta=173.4,158.2,153.1,152.5,146.2,134.0$, 127.7, 126.9, 114.1, 113.9, 113.5, 113.4, 83.1, 82.2, 81.8, 55.5, 51.8, 50.4, 33.3, 29.5, 28.3, 21.9 ppm.

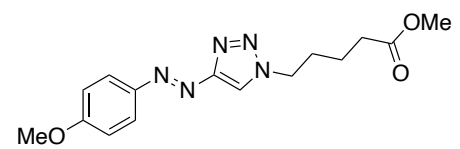

methyl (E)-5-(4-((4-methoxyphenyl)diazenyl)-1H-1,2,3-triazol-1-yl)pentanoate (16)

To a $0^{\circ} \mathrm{C}$ cooled solution of Di-tert-butyl 1-(1-(5-methoxy-5-oxopentyl)-1H-1,2,3-triazol-4-yl)-2-(4methoxyphenyl)hydrazine-1,2-dicarboxylate $(0.140 \mathrm{~g}, 0.27 \mathrm{mmol}, 1.0$ equiv) in DCM (13.5 $\mathrm{mL})$ was added $\mathrm{N}$-methylmorpholine $(0.148 \mathrm{~mL}, 1.345 \mathrm{mmol}, 5.0$ equiv), followed by the dropwise addition of trimethylsilyl iodide $(0.11 \mathrm{~mL}, 0.81 \mathrm{mmol}, 3.0$ equiv). The resulting solution was warmed to ambient temperature and stirred for $3 \mathrm{~h}$. The reaction vessel atmosphere was exchanged to $\mathrm{O}_{2}$. To the solution was added a saturated aqueous $\mathrm{NaHCO}_{3}$ solution $(6.75 \mathrm{~mL})$. The biphasic solution was stirred vigorously for $14 \mathrm{~h}$. To the solution was added DCM (15 mL) and $\mathrm{H}_{2} \mathrm{O}(20 \mathrm{~mL})$. The layers were separated, and the aqueous layer was further extracted with DCM $(2 \times 15 \mathrm{~mL})$. The organic extracts were combined, washed with brine $(20 \mathrm{~mL})$, dried over $\mathrm{Na}_{2} \mathrm{SO}_{4}$, filtered, and concentrated under reduced pressure. The residue was purified by chromatography on silica gel (2\% EtOAc in DCM to 5\% EtOAc in DCM) to afford compound 16 $(0.042 \mathrm{~g}, 49 \%)$ as a yellow solid.

${ }^{1} \mathrm{H}$ NMR $\left(400 \mathrm{MHz}, \mathrm{CDCl}_{3}\right) \delta=7.91-7.89(\mathrm{~m}, 3 \mathrm{H}), 6.97(\mathrm{~d}, J=9 \mathrm{~Hz}, 2 \mathrm{H}), 4.41(\mathrm{t}, J=7.1 \mathrm{~Hz}, 2 \mathrm{H})$, $3.85(\mathrm{~s}, 3 \mathrm{H}$ ), $3.64(\mathrm{~s}, 3 \mathrm{H}$ ), 2.35 (t, $J=7.2 \mathrm{~Hz}, 2 \mathrm{H}$ ), 2.00 (quint, $J=7.8 \mathrm{~Hz}, 2 \mathrm{H}$ ), 1.67 (quint, $J=7.6$ $\mathrm{Hz}, 2 \mathrm{H}) \mathrm{ppm}$.

${ }^{13} \mathrm{C}$ NMR $\left(100 \mathrm{MHz}, \mathrm{CDCl}_{3}\right) \delta=173.3,162.7,160.8,147.0,125.1,114.5,114.4,55.7,51.7,50.6$, 33.2, 29.5, $21.8 \mathrm{ppm}$.

HRMS (ES $\left.{ }^{+}\right): m / z$ [M + Na] $]^{+}$Calcd for $\mathrm{C}_{15} \mathrm{H}_{19} \mathrm{~N}_{5} \mathrm{O}_{3} \mathrm{Na}^{+} 340.1386$; Found 340.1395.

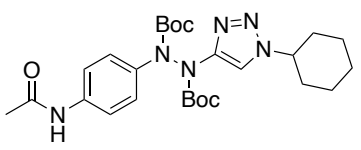

\section{di-tert-butyl 1-(4-acetamidophenyl)-2-(1-cyclohexyl-1H-1,2,3-triazol-4-yl)hydrazine-1,2- dicarboxylate}

To a solution of arylynehydrazide $4 \mathrm{e}(0.105 \mathrm{~g}, 0.270 \mathrm{mmol}, 1.0$ equiv) in THF $(0.54 \mathrm{~mL})$ was added cyclohexyl azide ${ }^{8}(0.041 \mathrm{~g}, 0.324 \mathrm{mmol}, 1.2$ equiv), triethylamine $(0.045 \mathrm{~mL}, 0.324 \mathrm{mmol}$, 1.2 equiv), and copper(I) iodide $(0.010 \mathrm{~g}, 0.05 \mathrm{mmol}, 0.2$ equiv). The mixture was stirred at ambient temperature for $24 \mathrm{~h}$, diluted with EtOAc $(10 \mathrm{~mL})$, and filtered through celite. The reaction vessel and filter cake were washed with additional EtOAc. The organic solution was concentrated under reduced pressure and purified by chromatography on silica gel (50\% EtOAc in hexanes) to afford di-tert-butyl 1-(4-acetamidophenyl)-2-(1-cyclohexyl-1H-1,2,3-triazol-4-yl)hydrazine-1,2dicarboxylate $(0.130 \mathrm{~g}, 94 \%)$ as a white semi-solid.

${ }^{1} \mathrm{H}$ NMR $\left(400 \mathrm{MHz}, \mathrm{CDCl}_{3}\right) \delta=8.44-8.30(\mathrm{~m}, 1 \mathrm{H}), 7.89-7.72(\mathrm{~m}, 1 \mathrm{H}), 7.48-7.26(\mathrm{~m}, 4 \mathrm{H}), 4.42-$ $4.28(\mathrm{~m}, 1 \mathrm{H}), 2.22-2.12(\mathrm{~m}, 2 \mathrm{H}), 2.06(\mathrm{~s}, 3 \mathrm{H}), 1.93-1.83(\mathrm{~m}, 2 \mathrm{H}), 1.78-1.64(\mathrm{~m}, 3 \mathrm{H}), 1.55-1.36$ $(\mathrm{m}, 18 \mathrm{H}), 1.32-1.16(\mathrm{~m}, 2 \mathrm{H}) \mathrm{ppm}$.

${ }^{13} \mathrm{C}$ NMR $\left(100 \mathrm{MHz}, \mathrm{CDCl}_{3}\right) \delta=168.9,152.8,145.7,136.7,125.5,124.9,120.2,119.8,112.6$, $111.6,83.6,83.2,82.7,82.1,60.7,33.4,33.4,28.2,25.2,24.4$ ppm. 


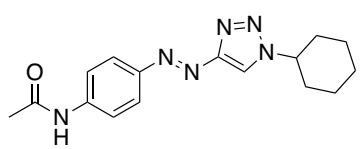

(E)-N-(4-((1-cyclohexyl-1H-1,2,3-triazol-4-yl)diazenyl)phenyl)acetamide (17)

To a $0^{\circ} \mathrm{C}$ cooled solution of Di-tert-butyl 1-(4-acetamidophenyl)-2-(1-cyclohexyl-1H-1,2,3-triazol4-yl)hydrazine-1,2-dicarboxylate $(0.130 \mathrm{~g}, 0.253 \mathrm{mmol}, 1.0$ equiv) in DCM (12.7 $\mathrm{mL})$ was added $\mathrm{N}$-methylmorpholine $(0.14 \mathrm{~mL}, 1.27 \mathrm{mmol}, 5.0$ equiv), followed by the dropwise addition of trimethylsilyl iodide $(0.11 \mathrm{~mL}, 0.76 \mathrm{mmol}, 3.0$ equiv). The resulting solution was warmed to ambient temperature and stirred for $3 \mathrm{~h}$. The reaction vessel atmosphere was exchanged to $\mathrm{O}_{2}$. To the solution was added a saturated aqueous $\mathrm{NaHCO}_{3}$ solution $(6.3 \mathrm{~mL})$. The biphasic solution was stirred vigorously for $20 \mathrm{~h}$. To the solution was added DCM $(20 \mathrm{~mL})$ and $\mathrm{H}_{2} \mathrm{O}(20 \mathrm{~mL})$. The layers were separated, and the aqueous layer further extracted with DCM $(2 \times 20 \mathrm{~mL})$. The organic extracts were combined, washed with brine $(20 \mathrm{~mL})$, dried over $\mathrm{Na}_{2} \mathrm{SO}_{4}$, filtered, and concentrated under reduced pressure. The residue was purified by chromatography on silica gel $(5 \%$ EtOAc in DCM to $20 \%$ EtOAc in DCM) to afford compound $17(0.028 \mathrm{~g}, 36 \%)$ as a yellow solid.

${ }^{1} \mathrm{H}$ NMR $\left(400 \mathrm{MHz}, \mathrm{DMSO}-d_{6}\right) \delta=10.31(\mathrm{~s}, 1 \mathrm{H}), 8.60(\mathrm{~s}, 1 \mathrm{H}), 7.85-7.77(\mathrm{~m}, 4 \mathrm{H}), 4.58-4.48(\mathrm{~m}$, $1 \mathrm{H}), 2.14-2.04(\mathrm{~m}, 5 \mathrm{H}), 1.87-1.78(\mathrm{~m}, 4 \mathrm{H}), 1.71-1.62(\mathrm{~m}, 1 \mathrm{H}), 1.50-1.36(\mathrm{~m}, 2 \mathrm{H}), 1.28-1.18(\mathrm{~m}$, 1H) ppm.

${ }^{13} \mathrm{C}$ NMR $\left(100 \mathrm{MHz}, \mathrm{DMSO}-d_{6}\right) \delta=168.9,159.9,147.6,142.6,123.5,119.2,114.0,59.9,32.6$, 24.6, $24.2 \mathrm{ppm}$.

HRMS $\left(\mathrm{ES}^{+}\right): \mathrm{m} / \mathrm{z}[\mathrm{M}+\mathrm{Na}]^{+}$Calcd for $\mathrm{C}_{16} \mathrm{H}_{20} \mathrm{~N}_{6} \mathrm{ONa}^{+}$335.1596; Found 335.1608.

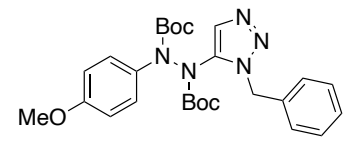

\section{di-tert-butyl 1-(1-benzyl-1H-1,2,3-triazol-5-yl)-2-(4-methoxyphenyl)hydrazine-1,2- dicarboxylate}

To a solution of arylynehydrazide $4 \mathrm{c}(0.107 \mathrm{~g}, 0.295 \mathrm{mmol}, 1.0$ equiv) in 1,4-dioxane $(1.5 \mathrm{~mL})$ was added benzyl azide ${ }^{3}\left(0.047 \mathrm{~g}, 0.355 \mathrm{mmol}, 1.2\right.$ equiv) and $\mathrm{Ru}(\mathrm{cp}) \mathrm{Cl}\left(\mathrm{PPh}_{3}\right)_{2}(0.011 \mathrm{~g}, 0.014$ mmol, 0.05 equiv). The mixture was stirred and heated at $80^{\circ} \mathrm{C}$ for $24 \mathrm{~h}$, then cooled to ambient temperature, diluted with EtOAc $(20 \mathrm{~mL})$, and filtered through celite. The reaction vessel and filter cake were washed with additional EtOAc. The organic solution was concentrated under reduced pressure and the residue was purified by chromatography on silica gel $(20 \%$ EtOAc in hexanes) to afford di-tert-butyl 1-(1-benzyl-1 H-1,2,3-triazol-5-yl)-2-(4-methoxyphenyl)hydrazine1,2-dicarboxylate $(0.094 \mathrm{~g}, 64 \%)$ as a brown oil.

${ }^{1} \mathrm{H}$ NMR $\left(400 \mathrm{MHz}, \mathrm{CDCl}_{3}\right)$ (rotamers observed) $\delta=7.70-7.63(\mathrm{~m}, 1 \mathrm{H}), 7.22-6.69(\mathrm{~m}, 9 \mathrm{H}), 5.35-$ $4.74(\mathrm{~m}, 2 \mathrm{H}), 3.78-3.76(\mathrm{~m}, 3 \mathrm{H}), 1.54-1.20(\mathrm{~m}, 18 \mathrm{H}) \mathrm{ppm}$.

${ }^{13} \mathrm{C}$ NMR $\left(100 \mathrm{MHz}, \mathrm{CDCl}_{3}\right)$ (rotamers observed) $\delta=158.9,153.2,152.7,136.9,136.7,134.6$, 134.2, 133.5, 130.4, 129.7, 128.6, 128.0, 127.9, 127.6, 127.2, 114.4, 114.0, 84.0, 83.1, 82.6, 55.4, $52.0,51.6,50.9,28.3,28.1,27.7 \mathrm{ppm}$.

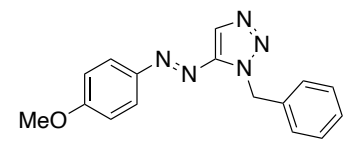

(E)-1-benzyl-5-((4-methoxyphenyl)diazenyl)-1H-1,2,3-triazole (18)

To a $0^{\circ} \mathrm{C}$ cooled solution of Di-tert-butyl 1-(1-benzyl-1H-1,2,3-triazol-5-yl)-2-(4methoxyphenyl)hydrazine-1,2-dicarboxylate $(0.061 \mathrm{~g}, 0.123 \mathrm{mmol}, 1.0$ equiv) in DCM $(6.2 \mathrm{~mL})$ was added $\mathrm{N}$-methylmorpholine $(0.068 \mathrm{~mL}, 0.616 \mathrm{mmol}, 5.0$ equiv) followed by the dropwise 
addition of trimethylsilyl iodide $(0.053 \mathrm{~mL}, 0.370 \mathrm{mmol}, 3.0$ equiv). The resulting solution was stirred at $40^{\circ} \mathrm{C}$ for $21 \mathrm{~h}$. The reaction vessel was cooled to ambient temperature, and the atmosphere was exchanged to $\mathrm{O}_{2}$. To the solution was added a saturated aqueous $\mathrm{NaHCO}_{3}$ solution $(3.1 \mathrm{~mL})$. The biphasic solution was stirred vigorously at ambient temperature for $23 \mathrm{~h}$. To the solution was added DCM $(30 \mathrm{~mL})$ and $\mathrm{H}_{2} \mathrm{O}(30 \mathrm{~mL})$. The layers were separated, and the aqueous layer was further extracted with DCM $(2 \times 30 \mathrm{~mL})$. The organic extracts were combined, washed with brine $(50 \mathrm{~mL})$, dried over $\mathrm{Na}_{2} \mathrm{SO}_{4}$, filtered, and concentrated under reduced pressure. The residue was purified by chromatography on silica gel (25\% EtOAc in hexanes) to afford compound $18(0.028 \mathrm{~g}, 78 \%)$ as a yellow solid.

${ }^{1} \mathrm{H}$ NMR $\left(400 \mathrm{MHz}, \mathrm{CDCl}_{3}\right) \delta=7.93(\mathrm{~s}, 1 \mathrm{H}), 7.86(\mathrm{~d}, J=9.0 \mathrm{~Hz}, 2 \mathrm{H}), 7.38-7.28(\mathrm{~m}, 5 \mathrm{H}), 7.01$ (d, $J=9.0 \mathrm{~Hz}, 2 \mathrm{H}), 5.89(\mathrm{~s}, 2 \mathrm{H}), 3.90(\mathrm{~s}, 3 \mathrm{H}) \mathrm{ppm}$.

${ }^{13} \mathrm{C}$ NMR $\left(100 \mathrm{MHz}, \mathrm{CDCl}_{3}\right) \delta=163.6,147.2,135.3,129.0,128.5,128.5,128.2,125.5,122.5$, 114.7, 55.8, $52.0 \mathrm{ppm}$.

HRMS $\left(\mathrm{ES}^{+}\right): \mathrm{m} / \mathrm{z}[\mathrm{M}+\mathrm{H}]^{+}$Calcd for $\mathrm{C}_{16} \mathrm{H}_{16} \mathrm{~N}_{5} \mathrm{O}^{+}$294.1355; Found 294.1367

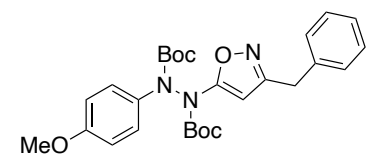

di-tert-butyl 1-(3-benzylisoxazol-5-yl)-2-(4-methoxyphenyl)hydrazine-1,2-dicarboxylate

To a $0^{\circ} \mathrm{C}$ cooled solution of arylynehydrazide $4 \mathrm{c}(0.200 \mathrm{~g}, 0.552 \mathrm{mmol}, 1.0$ equiv) in THF (2.68 $\mathrm{mL}$ ) was added (Z)-N-hydroxy-2-phenylacetimidoyl chloride ${ }^{10}(0.187 \mathrm{~g}, 1.104 \mathrm{mmol}, 2.0$ equiv), and copper $(\mathrm{I})$ iodide $(0.021 \mathrm{~g}, 0.110 \mathrm{mmol}, 0.2$ equiv). To the mixture was added a solution of triethylamine $(0.17 \mathrm{~mL}, 1.214 \mathrm{mmol}, 2.2$ equiv) in THF (1 mL) dropwise over 30 minutes. The mixture was warmed to ambient temperature, stirred for $21 \mathrm{~h}$, and filtered through celite. The reaction vessel and filter cake were washed with additional EtOAc. To the organic solution was added $\mathrm{H}_{2} \mathrm{O}(30 \mathrm{~mL})$ and the layers were separated. The aqueous layer was further extracted with EtOAc $(2 \times 30 \mathrm{~mL})$. The organic extracts were combined, washed with brine $(50 \mathrm{~mL})$, dried over $\mathrm{Na}_{2} \mathrm{SO}_{4}$, filtered, and concentrated under reduced pressure. The residue was purified by chromatography on silica gel (9\% EtOAc in hexanes) to afford di-tert-butyl 1-(3-benzylisoxazol-5yl)-2-(4-methoxyphenyl)hydrazine-1,2-dicarboxylate $(0.157 \mathrm{~g}, 58 \%)$ as a colorless oil.

${ }^{1} \mathrm{H}$ NMR $\left(400 \mathrm{MHz}, \mathrm{CDCl}_{3}\right)$ (rotamers observed) $\delta=7.47-7.34(\mathrm{~m}, 2 \mathrm{H}), 7.33-7.19(\mathrm{~m}, 5 \mathrm{H}), 6.89$ $6.80(\mathrm{~m}, 2 \mathrm{H}), 6.22$ - $5.22(\mathrm{~m}, 1 \mathrm{H}), 4.00-3.88(\mathrm{~m}, 2 \mathrm{H}), 3.80-3.76(\mathrm{~m}, 3 \mathrm{H}), 1.56-1.38(\mathrm{~m}, 18 \mathrm{H}) \mathrm{ppm}$. ${ }^{13} \mathrm{C}$ NMR $\left(100 \mathrm{MHz}, \mathrm{CDCl}_{3}\right)$ (rotamers observed) $\delta=165.0,164.8,158.5,152.6,150.4,137.0$, 133.8, 133.2, 129.0, 128.9, 128.8, 128.6, 127.5, 126.9, 114.1, 113.8, 89.5, 84.4, 82.8, 82.6, 55.5, $55.5,33.1,28.2,28.1,28.1,28.0$ ppm.

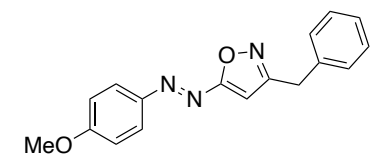

(E)-3-benzyl-5-((4-methoxyphenyl)diazenyl)isoxazole (19)

To a $0^{\circ} \mathrm{C}$ cooled solution of Di-tert-butyl 1-(3-benzylisoxazol-5-yl)-2-(4-methoxyphenyl)hydrazine1,2-dicarboxylate $(0.078 \mathrm{~g}, 0.157 \mathrm{mmol}, 1.0$ equiv) in DCM $(7.9 \mathrm{~mL})$ was added $\mathrm{N}$ methylmorpholine ( $0.087 \mathrm{~mL}, 0.787 \mathrm{mmol}, 5.0$ equiv) followed by the dropwise addition of trimethylsilyl iodide $\left(0.067 \mathrm{~mL}, 0.472 \mathrm{mmol}, 3.0\right.$ equiv). The resulting solution was stirred at $40^{\circ} \mathrm{C}$ for $24 \mathrm{~h}$. The reaction vessel was cooled to ambient temperature and the atmosphere was exchanged to $\mathrm{O}_{2}$. To the solution was added a saturated aqueous $\mathrm{NaHCO}_{3}$ solution $(3.9 \mathrm{~mL})$. The biphasic solution was stirred vigorously at ambient temperature for $27 \mathrm{~h}$. To the solution was 
added DCM (30 mL) and $\mathrm{H}_{2} \mathrm{O}(30 \mathrm{~mL})$. The layers were separated, and the aqueous layer was further extracted with DCM $(2 \times 30 \mathrm{~mL})$. The organic extracts were combined, washed with brine $(50 \mathrm{~mL})$, dried over $\mathrm{Na}_{2} \mathrm{SO}_{4}$, filtered, and concentrated under reduced pressure. The residue was purified by chromatography on silica gel (9\% EtOAc in hexanes) to afford compound 19 (0.020 g, $44 \%)$ as an orange solid.

${ }^{1} \mathrm{H}$ NMR $\left(400 \mathrm{MHz} \mathrm{CDCl}_{3}\right) \delta=7.94(\mathrm{~d}, J=9.1 \mathrm{~Hz}, 2 \mathrm{H}), 7.35-7.22(\mathrm{~m}, 5 \mathrm{H}), 7.01(\mathrm{~d}, J=9.1 \mathrm{~Hz}$, 2H), 6.40 (s, 1H), 4.08 (s, 2H), 3.90 (s, 3H) ppm.

${ }^{13} \mathrm{C}$ NMR $\left(100 \mathrm{MHz}, \mathrm{CDCl}_{3}\right) \delta=174.3,164.8,164.1,147.3,137.0,129.0,129.0,127.2,126.2$, 114.7, 96.5, 55.9, $33.2 \mathrm{ppm}$.

HRMS $\left(\mathrm{ES}^{+}\right): \mathrm{m} / \mathrm{z}[\mathrm{M}+\mathrm{H}]^{+}$Calcd For $\mathrm{C}_{17} \mathrm{H}_{16} \mathrm{~N}_{3} \mathrm{O}_{2}{ }^{+}$294.1423; Found 294.1361

\section{UV-Vis Characterization}

UV-Vis spectra for compounds $\mathbf{8 a}, \mathbf{8 b}, \mathbf{8 c}, \mathbf{8 d}, \mathbf{8 e}, \mathbf{1 8}$, and 19 were recorded on a Nanodrop 2000c spectrophotometer. Samples were dissolved in DMSO (3 mL, $50 \mu \mathrm{M}$ final concentration) and placed in a quartz cuvette. Solutions were illuminated by a Mightex WheeLED light source equipped with a fiber optic cable for $10 \mathrm{~min}$ at each successive wavelength at ambient temperature with stirring. Illumination and stirring were ceased momentarily while recording spectra.

UV-Vis for compounds 10,13 , and 14 were collected using the same method as above, and a comparison of the spectral properties of $\mathbf{8 c}, \mathbf{1 0}, \mathbf{1 3}$, and 14 is shown in Figure S1.

A
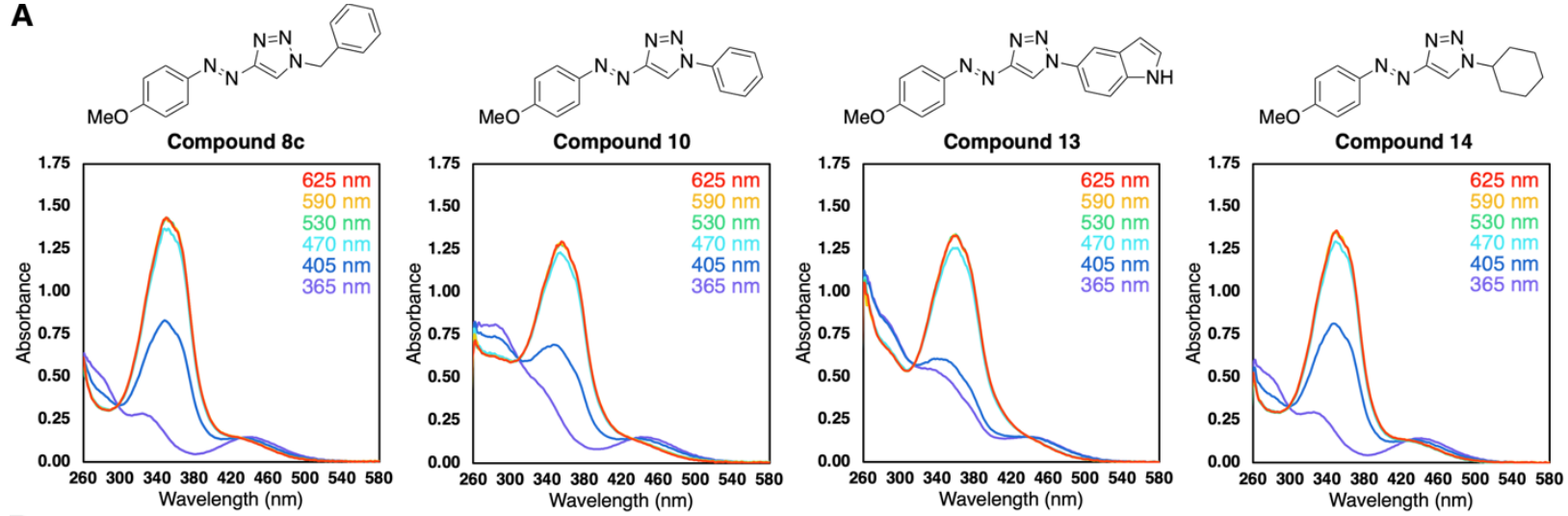

B

Photoswitch

$8 \mathrm{c}$

10

13

14

$E$ isomer $\pi \rightarrow \pi$
$352 \mathrm{~nm}$
$355 \mathrm{~nm}$
$358 \mathrm{~nm}$
$350 \mathrm{~nm}$

$Z$ isomer $n \rightarrow \pi$

$442 \mathrm{~nm}$

$441 \mathrm{~nm}$

$437 \mathrm{~nm}$

$438 \mathrm{~nm}$

Figure S1. (A) UV-Vis spectra of photoswitches prepared from modular arylynehydrazide $4 \mathrm{c}$ including $1^{\circ}$ alkyl (8c), aromatic (10), heteroaromatic (13), and $2^{\circ}$ alkyl (14) linked groups (50 $\mu \mathrm{M}$ in DMSO). (B) Photophysical properties of 8c, 10, 13, and 14 as determined by UV-Vis spectroscopy (electronic transitions).

\section{Constant Illumination ${ }^{1} \mathrm{H}$ NMR Experiments}

Compounds were dissolved in DMSO- $d_{6}$ with the sample volume and concentration being $450 \mu \mathrm{L}$ and $100 \mu \mathrm{M}$ respectively. Samples were illuminated using a Mightex WheeLED light source equipped with a fiber optic cable fed directly into the NMR tube. The tip of the fiber optic cable was placed just above the solvent, and the sample manually lowered into a $600 \mathrm{MHz} \mathrm{NMR}$ 
instrument. Ratios of isomers were measured after 10 min of illumination at $365 \mathrm{~nm}, 405 \mathrm{~nm}, 470$ $\mathrm{nm}, 530 \mathrm{~nm}, 590 \mathrm{~nm}$, and $625 \mathrm{~nm}$ successively for model photoswitches 8a, 8b, 8c, 8d, 8e, 18, and 19. Compounds $9,10,11,12,13,14,16$, and 17 were illuminated for 10 min at $365 \mathrm{~nm}$ and then $530 \mathrm{~nm}$, with NMR spectra collected at each wavelength. $365 \mathrm{~nm}$ and $470 \mathrm{~nm}$ were used for Compound 15.

\section{Thermal Stability and Half-life Measurements}

Thermal stability measurements for compounds $8 \mathrm{c}$ and $8 \mathrm{e}$ were assessed via ${ }^{1} \mathrm{H}$ NMR. Compounds were dissolved in DMSO- $d_{6}$ with the sample volume and concentration being $450 \mu \mathrm{L}$ and $100 \mu \mathrm{M}$ respectively. Samples were illuminated using a Mightex WheeLED light source equipped with a fiber optic cable fed directly into the NMR tube. The tip of the fiber optic cable was placed just above the solvent, the sample manually lowered into a $600 \mathrm{MHz} N M R$ instrument, and illuminated at $365 \mathrm{~nm}$ for 10 min to convert the compounds to predominantly the $Z$ isomer. An initial measurement was taken, then the sample removed in the dark and stored for 1 week before recording a final measurement. Thermal Stability indicates the percentage change of $Z$ isomer that reverts to the $E$ isomer after 1 week in the dark.

Thermal half-lives for compounds 18 and 19 were assessed via UV-Vis on a Nanodrop 2000c spectrophotometer. Samples were dissolved in DMSO (3 mL, $50 \mu \mathrm{M}$ final concentration) and placed in a quartz cuvette. Solutions were illuminated by a Mightex WheeLED light source equipped with a fiber optic cable for $10 \mathrm{~min}$ at $365 \mathrm{~nm}$ to convert the compounds to predominantly the $Z$ isomers. The light was removed, and the samples were monitored over an extended period of time for conversion to the $E$ isomer. Absorbances at $369 \mathrm{~nm}$ and $382 \mathrm{~nm}$ for compounds 18 and 19 respectively, were plotted versus time using GraphPad Prism 9.0.0 and fit using a one phase decay non-linear regression to determine half-lives.

\section{Supplemental References}

1. Ling, K. B.; Smith, A. D. a-Aroyloxyaldehydes: Scope and Limitations as Alternative to $\alpha-$ haloaldehydes for NHC-Catalysed Redox Transformations. Chem. Commun. 2011, 46, 373375.

2. Nguyen, S. S.; Ferreira, A. J.; Long, Z. G.; Heiss, T. K.; Dorn, R. S.; Row, R. D.; Prescher, J. A. Butenolide Synthesis from Functionalized Cyclopropenones. Org. Lett. 2019, 21, 21, 86958699.

3. Campbell-Verduyn, L. S.; Mirfeizi, L.; Dierckx, R. A.; Elsinga, P. H.; Feringa, B. L. Phosphoramadite Accelerated Copper(I)-catalyzed [3+2] cycloadditions of azides and alkynes. Chem. Commun. 2009, 2139-2141.

4. Gallina, M. E.; Baytekin, B.; Schalley, C.; Ceroni, P. Light-Harvesting in Multichromophoric Rotaxanes. Chem. Eur. J. 2012, 18, 1528-1535.

5. Mangione, M.; Spanavello, R. A.; Anzardi, M. B. Efficient and Straightforward Click Synthesis of Structurally Related Dendritic Triazoles. RSC Adv. 2017, 7, 47681-47688.

6. Wilkening, I.; del Signore, G.; Hackenberger, C. P. R. Synthesis of phosphonamidate peptides by Staudinger reactions of silylated phosphinic acids and esters. Chem. Commun. 2011, 47, 349-351.

7. Samadder, P.; Xu, Y.; Schweizer, F.; Arthur, G. Cytotoxic properties of D-gluco-, D-galactoand D-manno-configured 2-amino-2-deoxy-glycerolipids against epithelial cancer cell lines and BT-474 breast cancer stem cells. E. Journal Med. Chem. 2014, 78, 248-258. 
8. Maury, J.; Feray, L.; Bertrand, M.; Kapat, A.; Renaud, P. Unexpected Conversion of Alkyl Azides to Alkyl lodides and of Aryl Azides to N-tert-butyl Anilines. Tetrahedron. 2012, 68, 47, 9606-9611.

9. Schibilla, F.; Voskuhl, J.; Fokina, N. A.; Dahl, J. E. P.; Schreiner, P. R.; Ravoo, B. J. HostGuest Complexex of Cyclodextrins and Nanodiamonds as a Strong Non-Covalent Binding Motif for Self-Assembled Nanomaterials. Chem. Eur. J. 2017, 23, 63, 16059-16065.

10. Vo, Q. V.; Trenerry, C.; Rochfort, S.; Wadeson, J.; Leyton, C.; Hughes, A. B. Synthesis and anti-inflammatory activity of aromatic glucosinolates Bioorg. Med. Chem. 2013, 21, 59455954. 


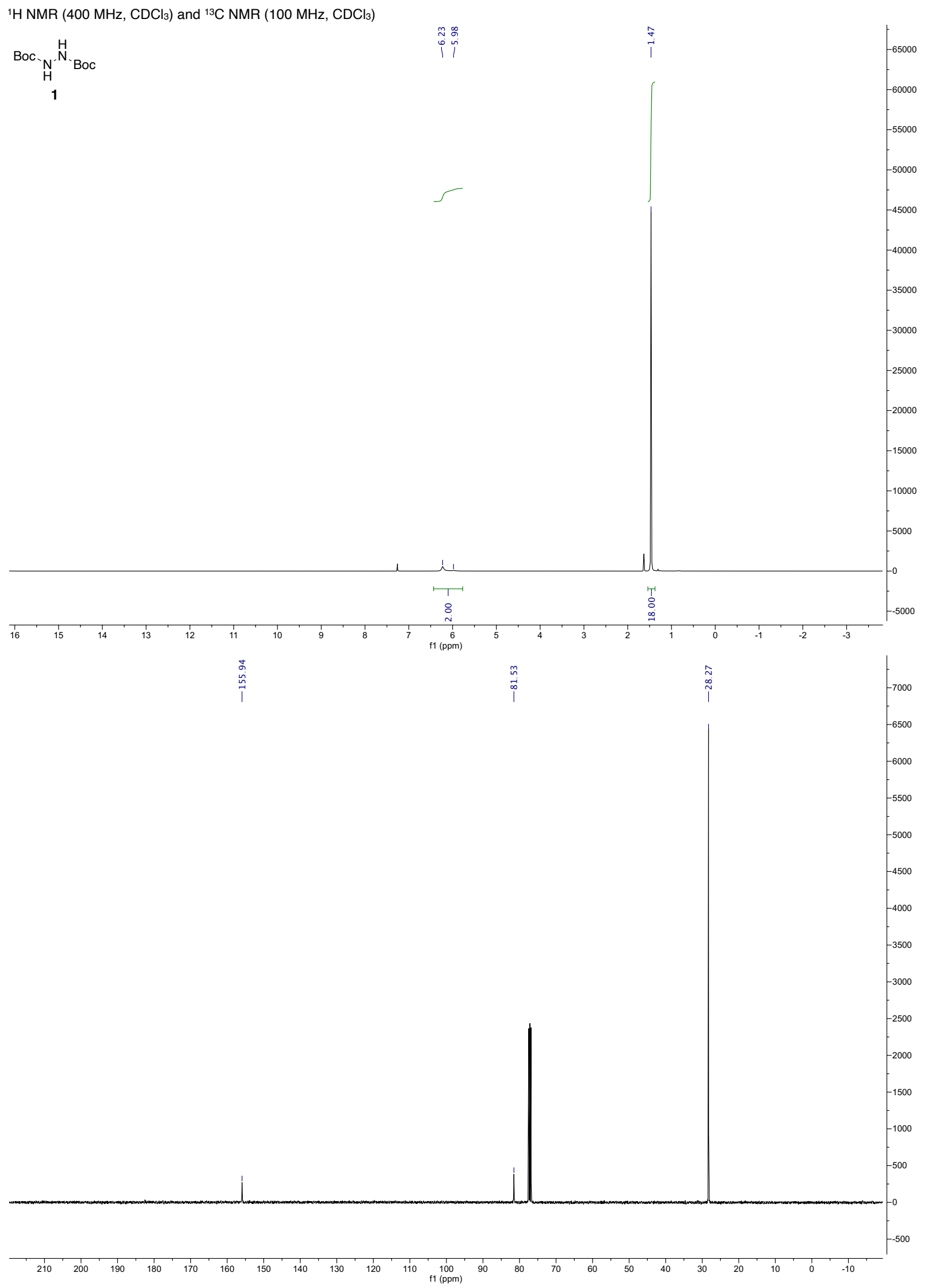




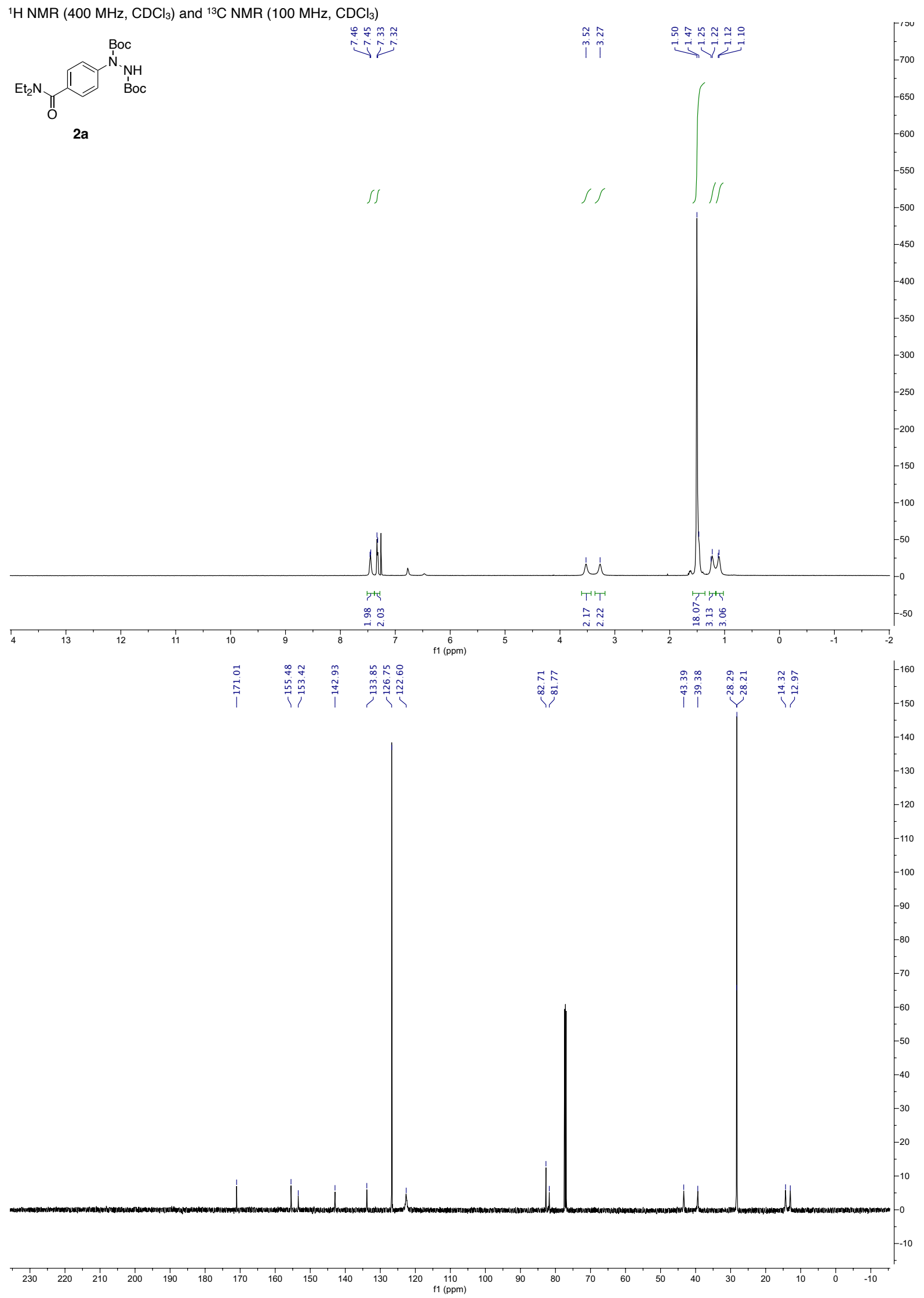




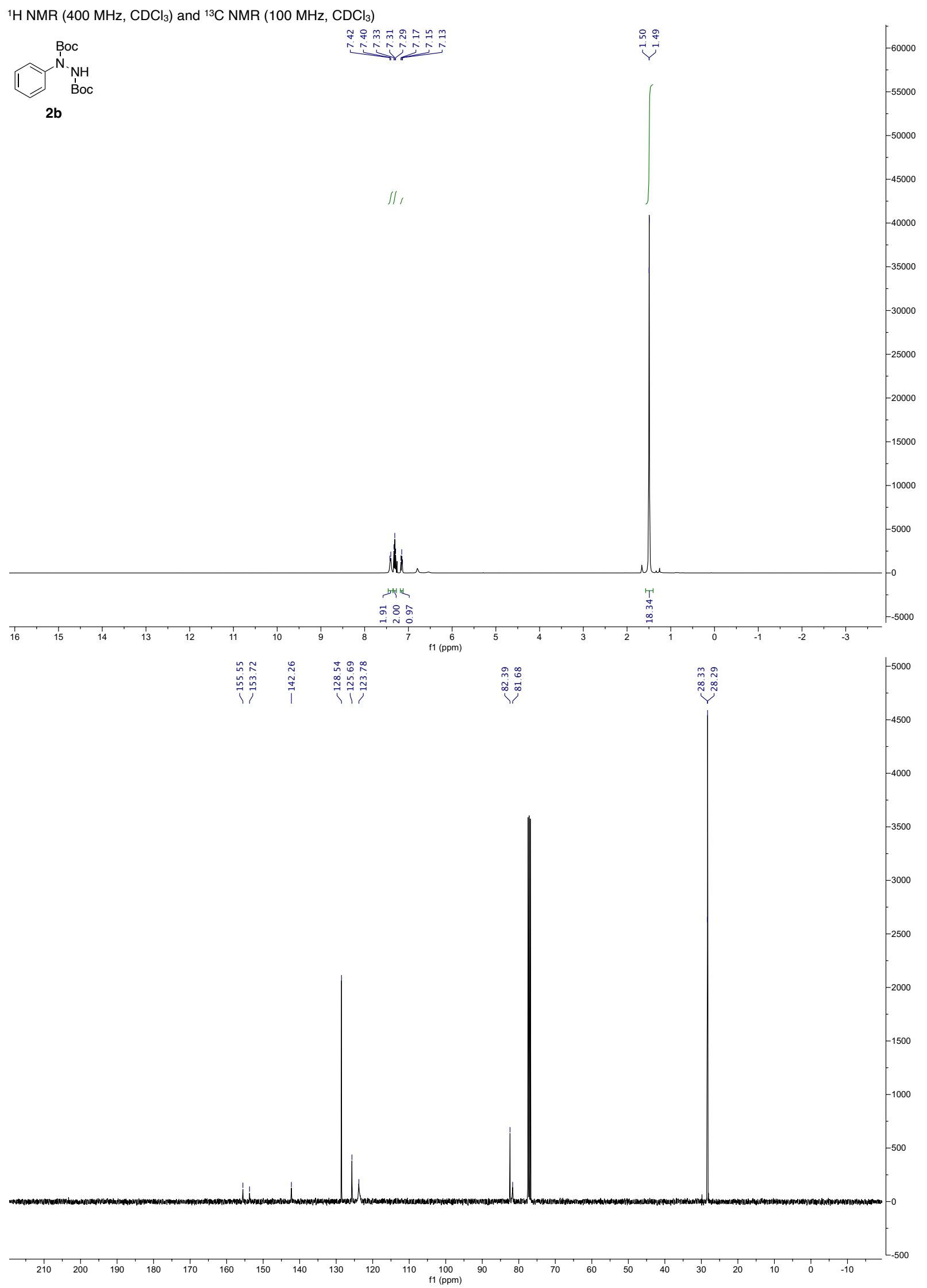




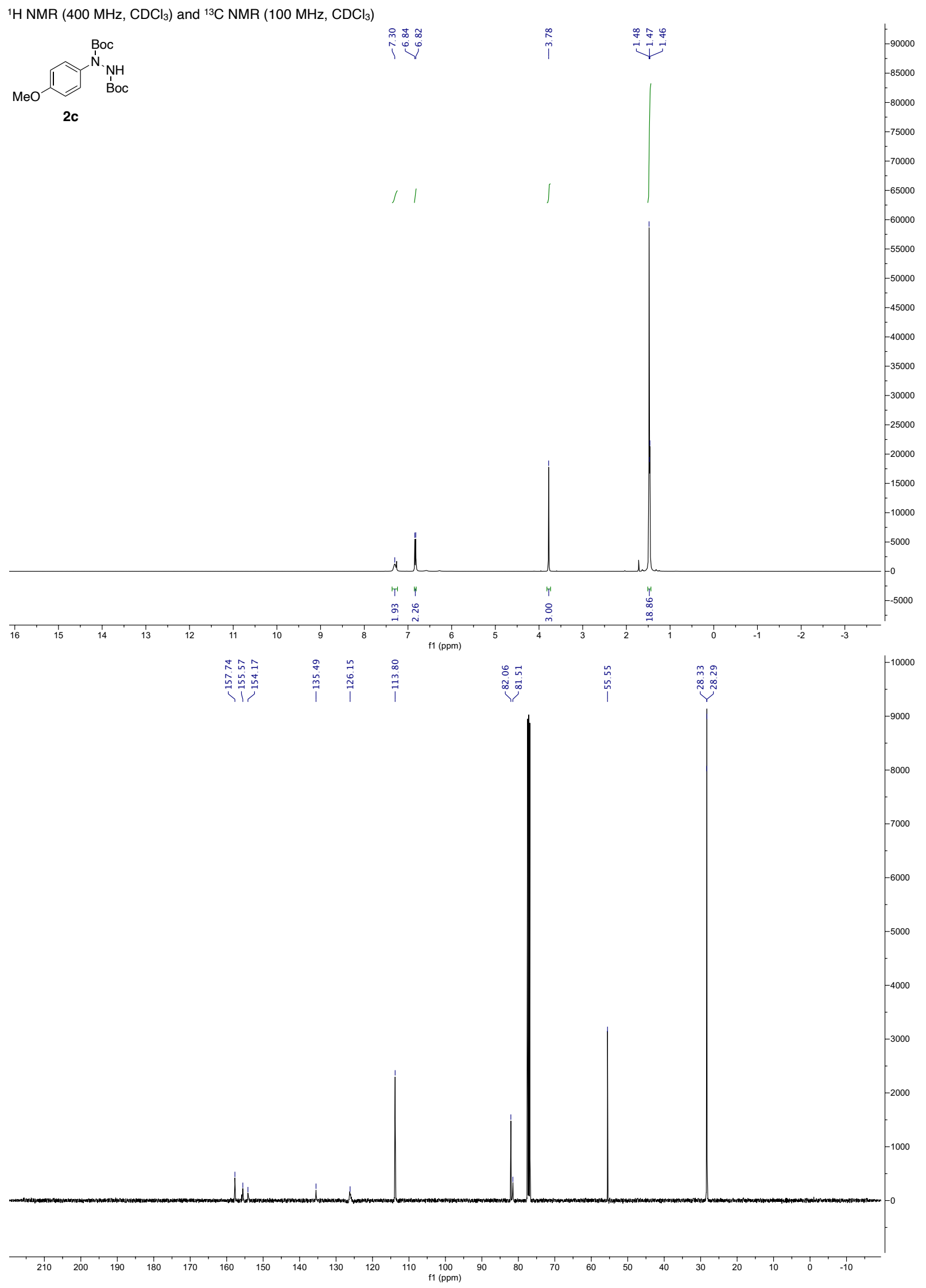




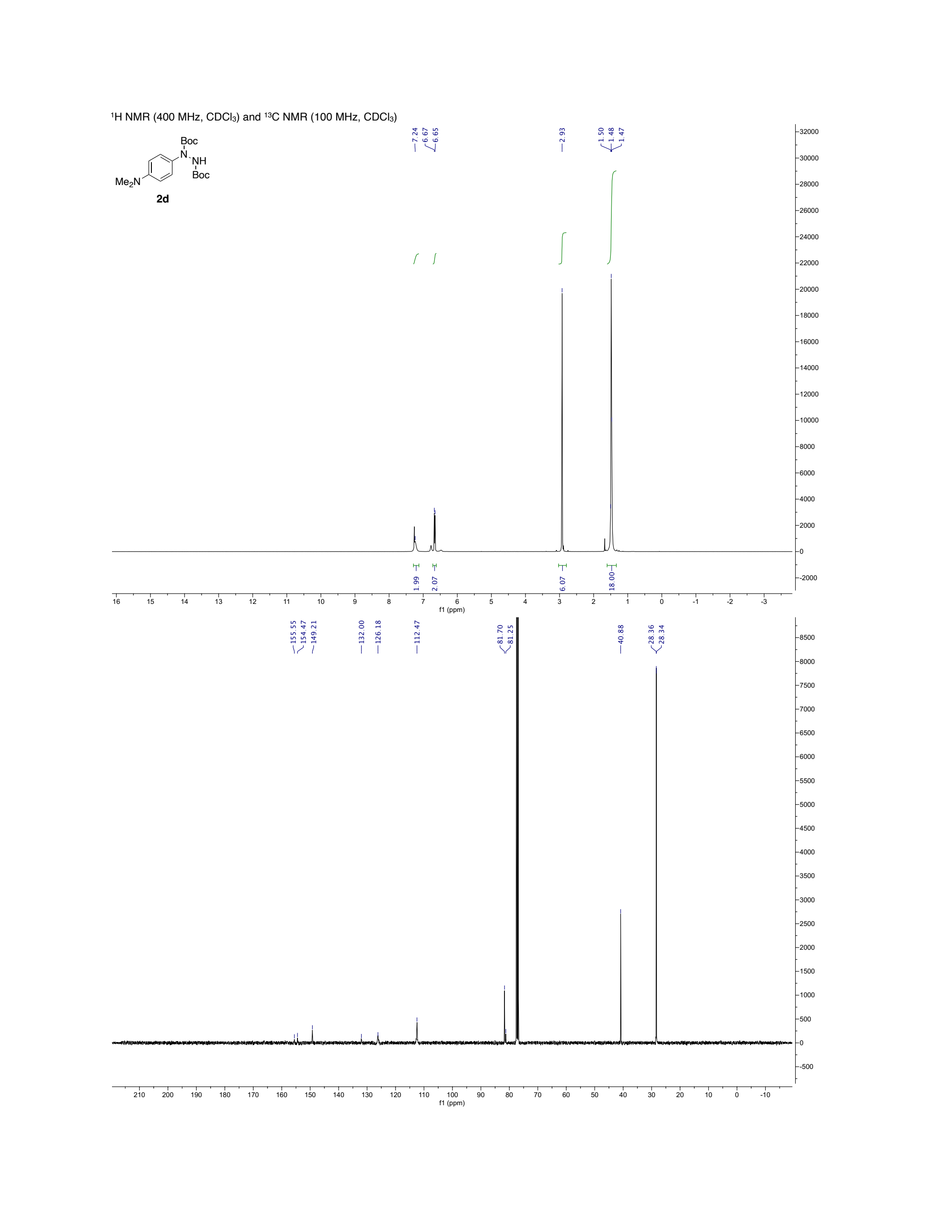




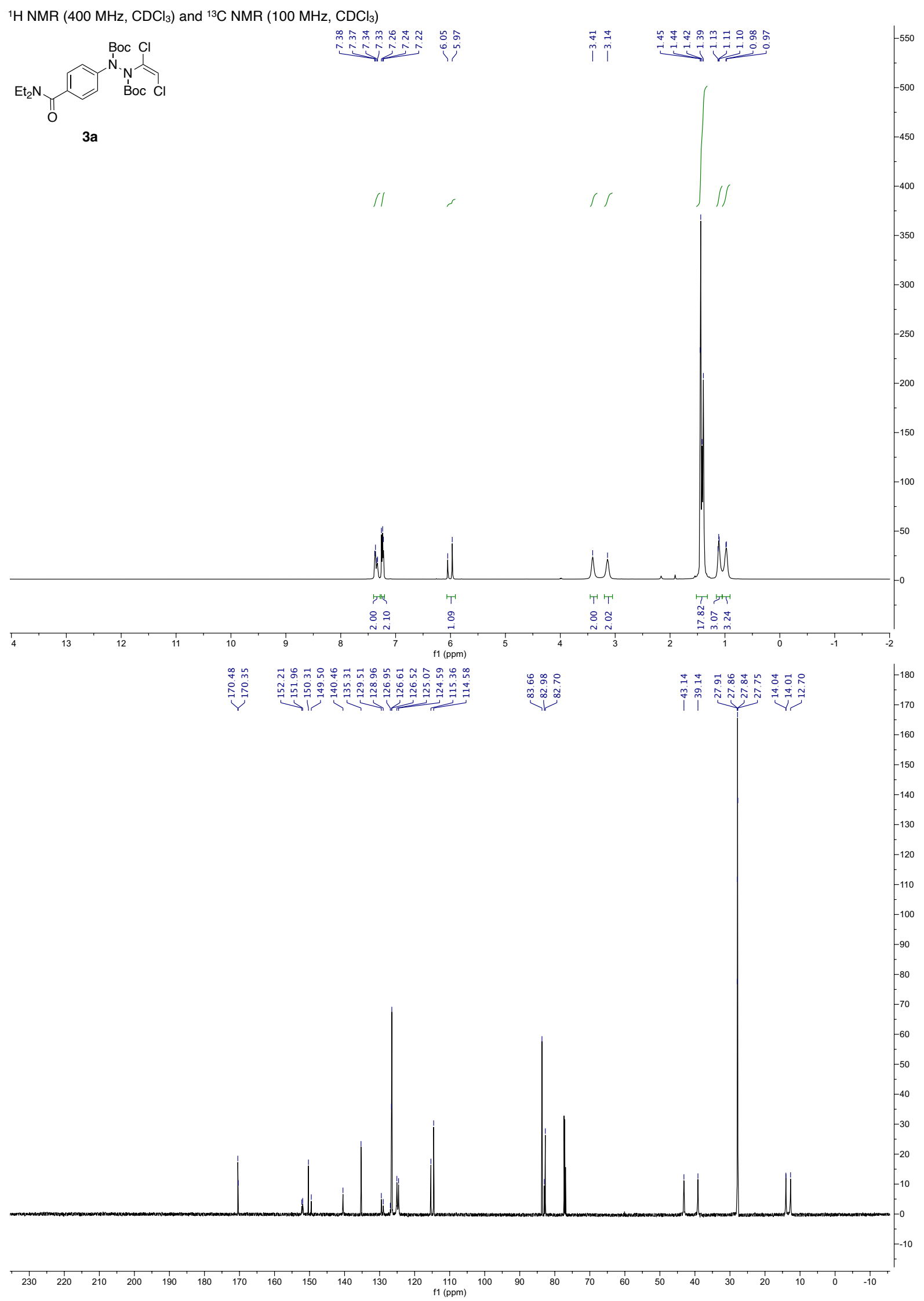


${ }^{1} \mathrm{H}$ NMR (400 MHz, CDCl 3 ) and $\left.{ }^{13} \mathrm{C} \mathrm{NMR} \mathrm{(100} \mathrm{MHz,} \mathrm{CDCl}_{3}\right)$
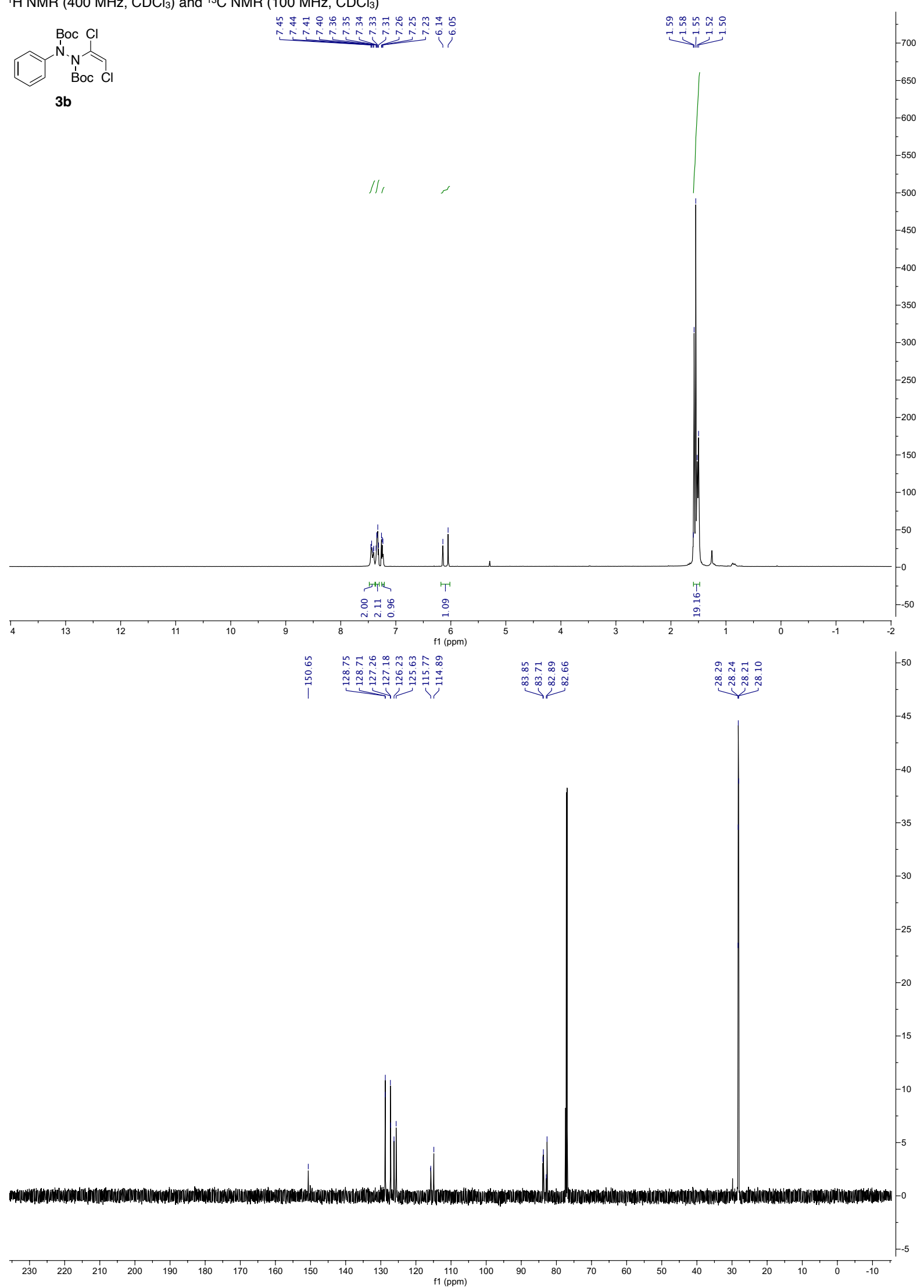
${ }^{1} \mathrm{H} \mathrm{NMR}\left(400 \mathrm{MHz}, \mathrm{CDCl}_{3}\right)$ and ${ }^{13} \mathrm{C} \mathrm{NMR}\left(100 \mathrm{MHz}, \mathrm{CDCl}_{3}\right)$

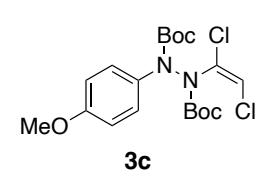

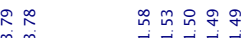

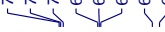

$n_{m}^{\infty}$

souvu

$-34000$

$-32000$

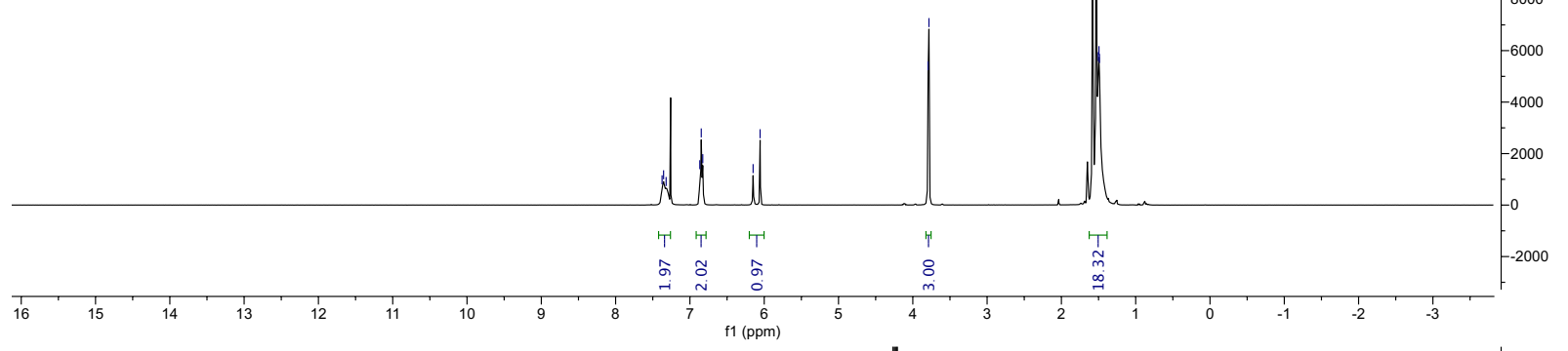

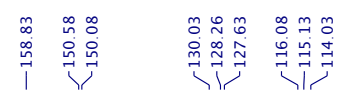
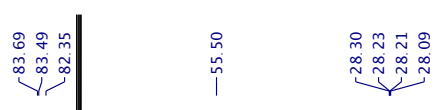

$-6000$ 
${ }^{1} \mathrm{H}$ NMR (400 MHz, $\left.\mathrm{CDCl}_{3}\right)$ and $\left.{ }^{13} \mathrm{C} \mathrm{NMR} \mathrm{(100} \mathrm{MHz,} \mathrm{CDCl}_{3}\right)$

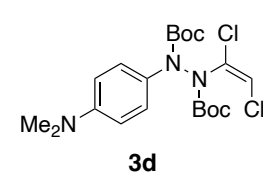

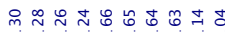

氷药

$3 d$

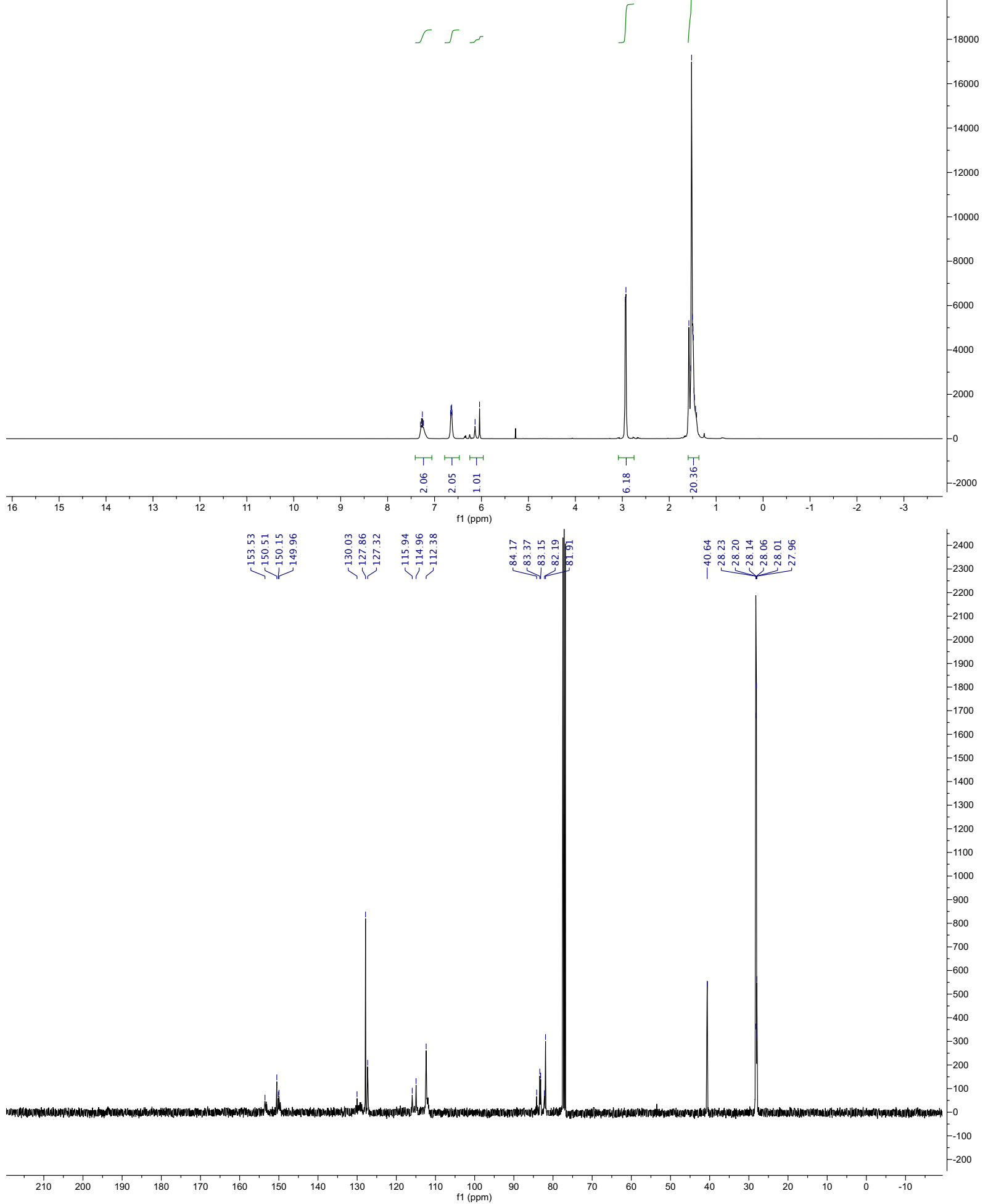




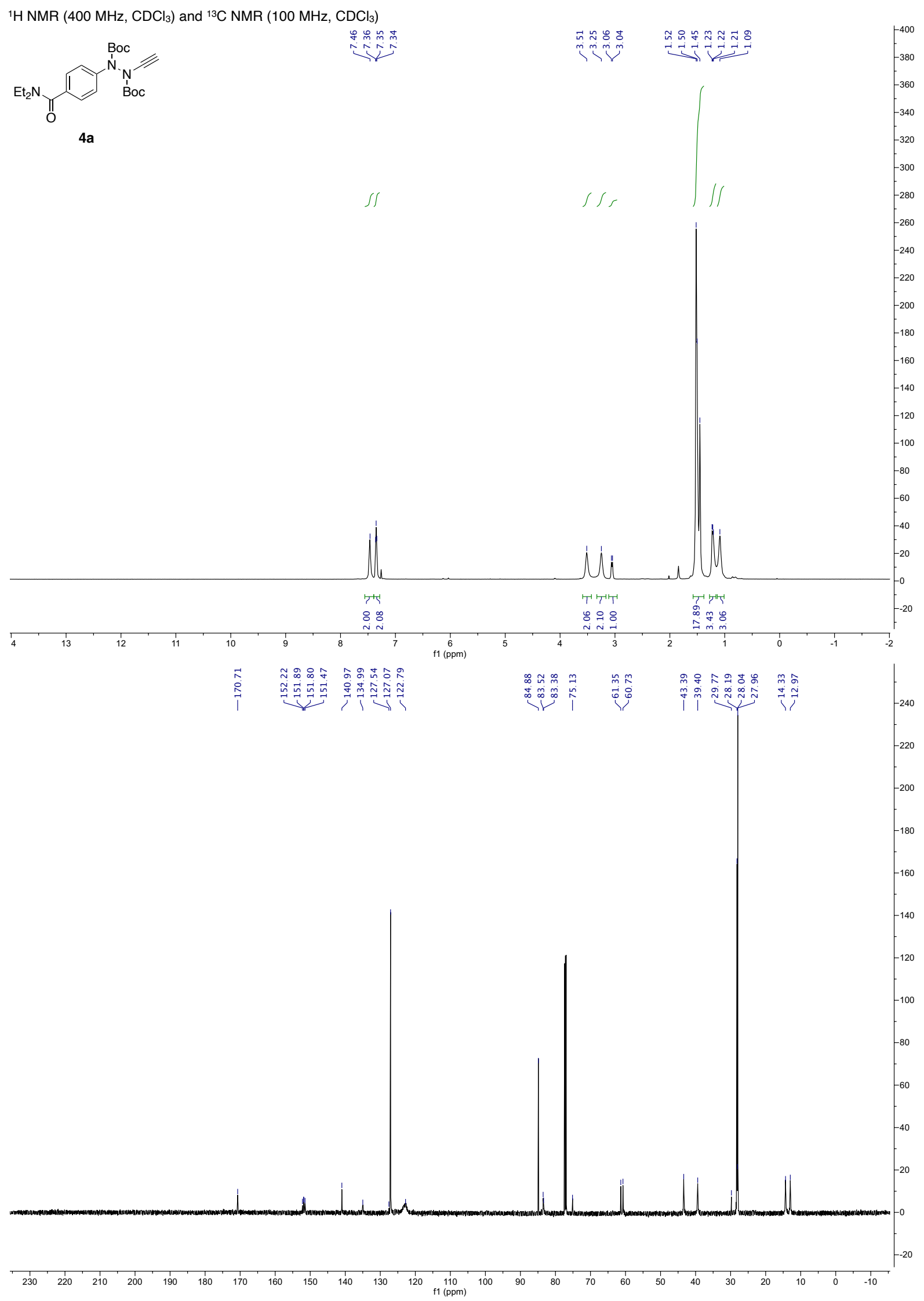




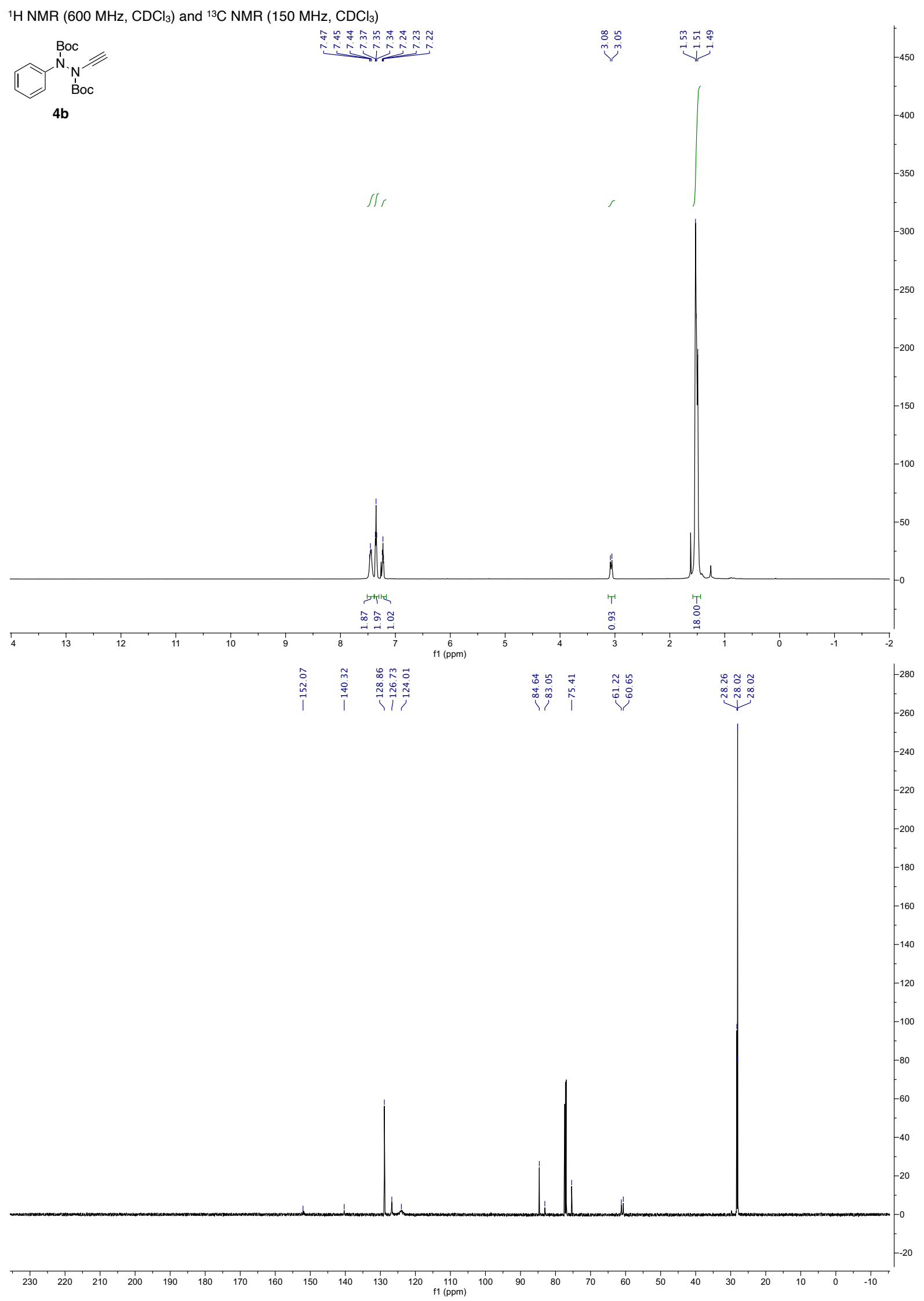




$$
=
$$




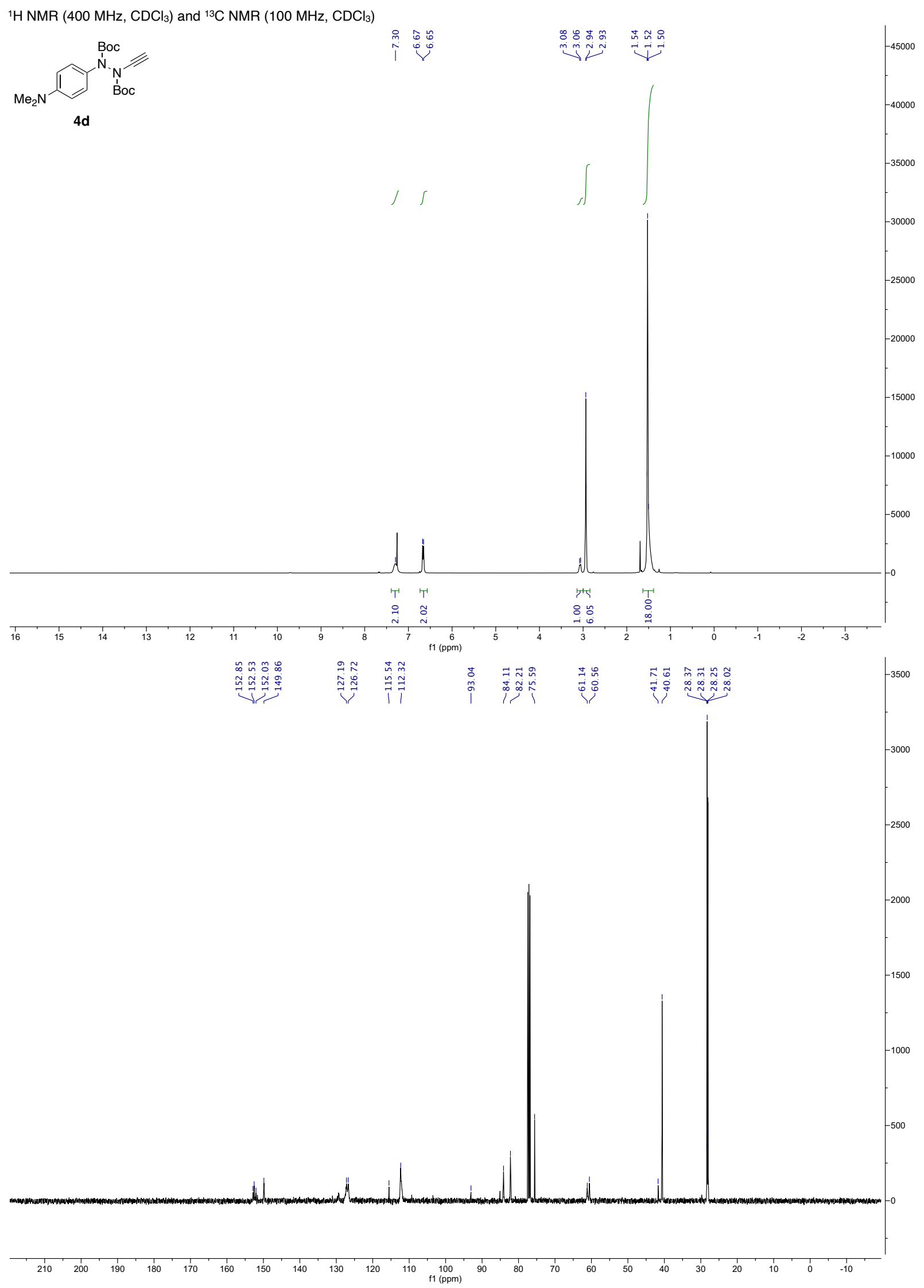




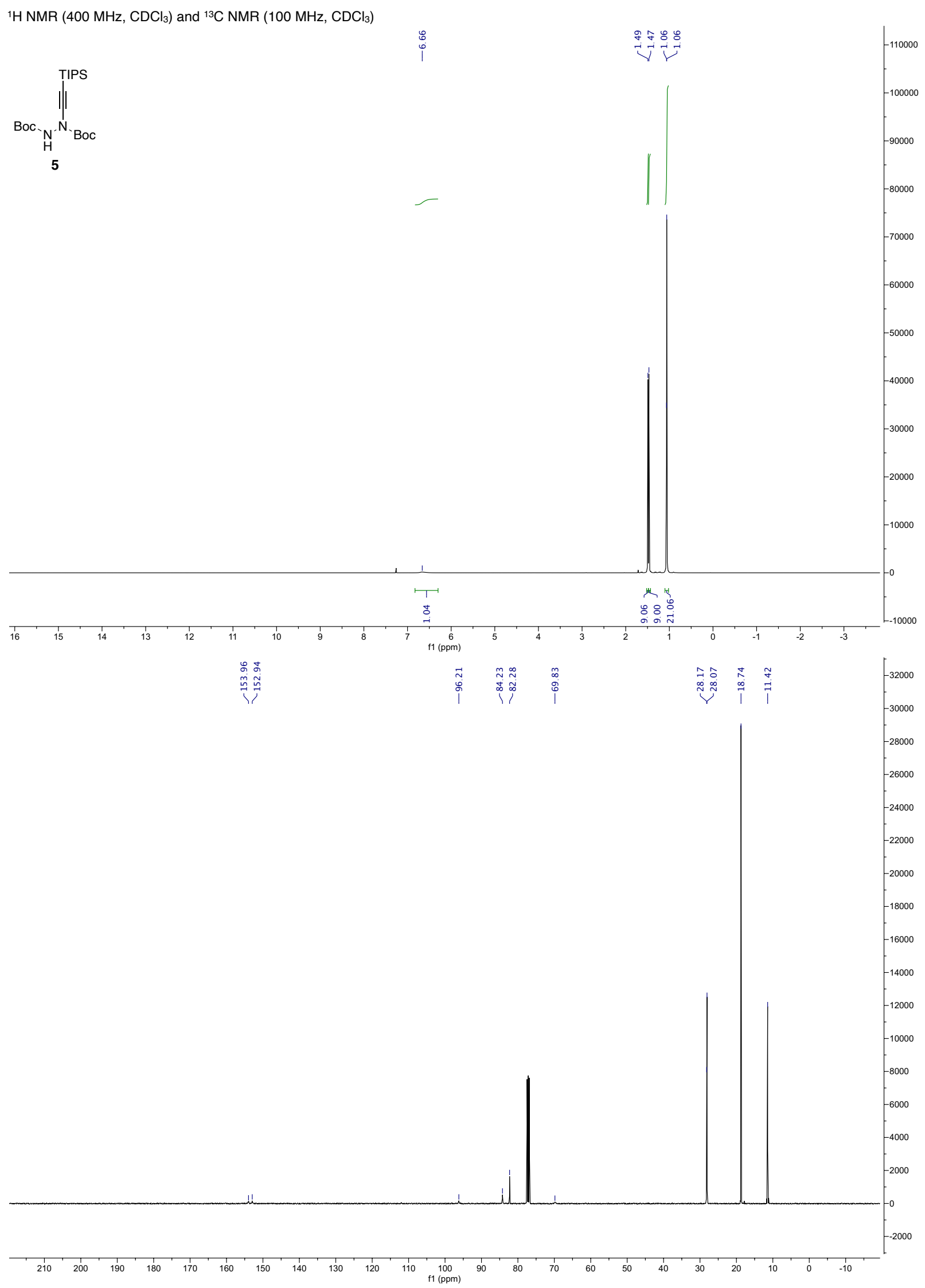




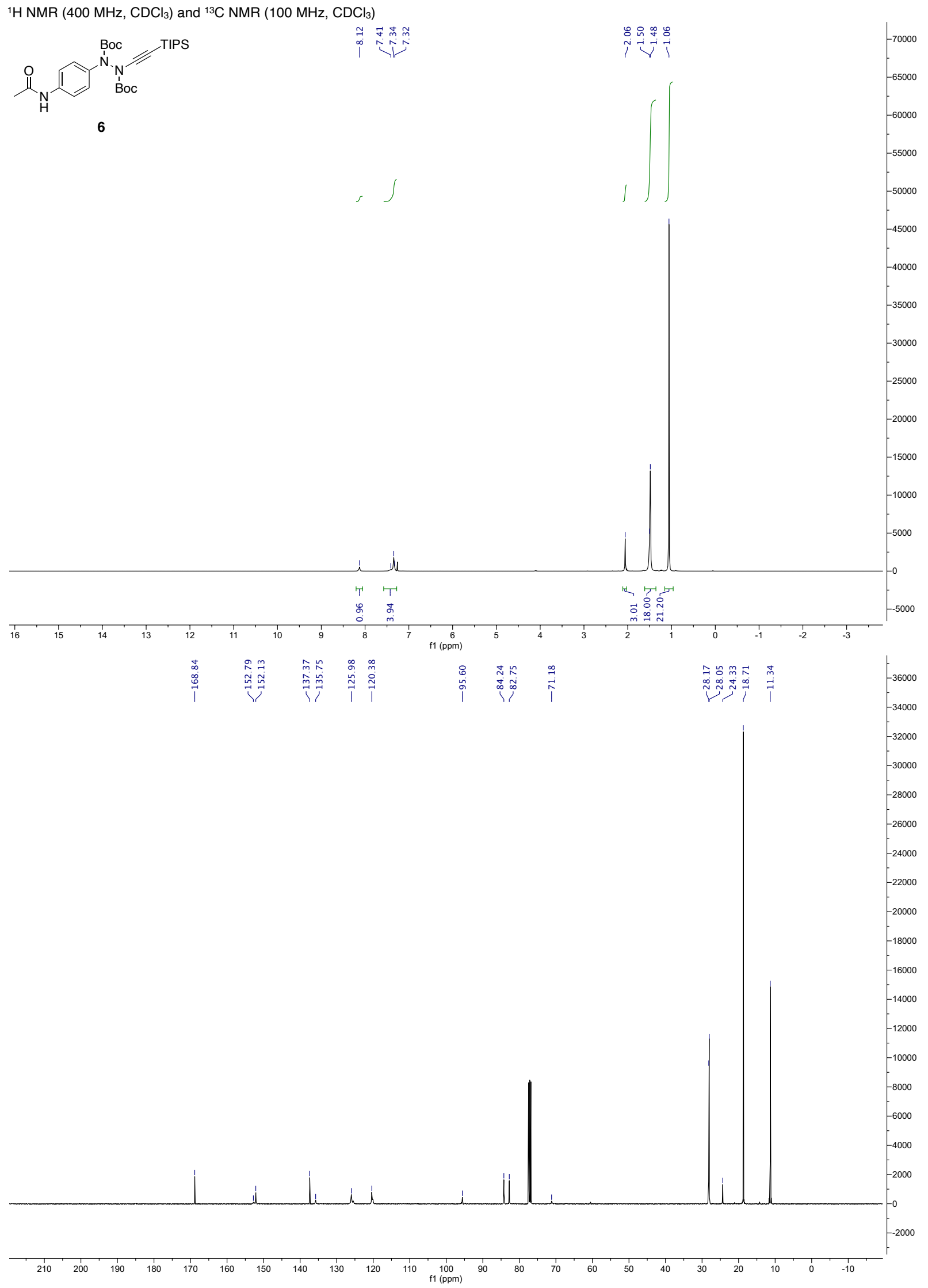




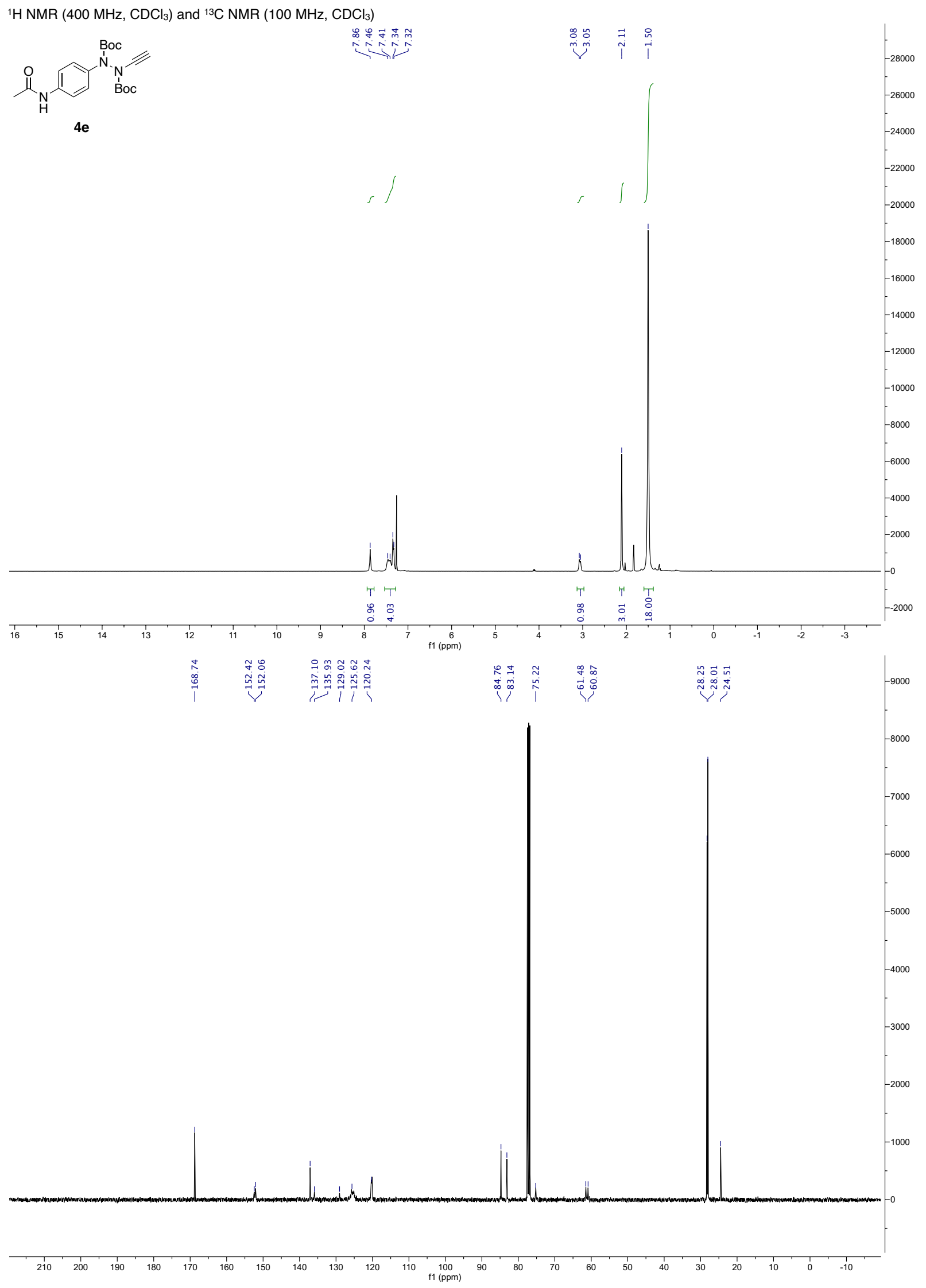




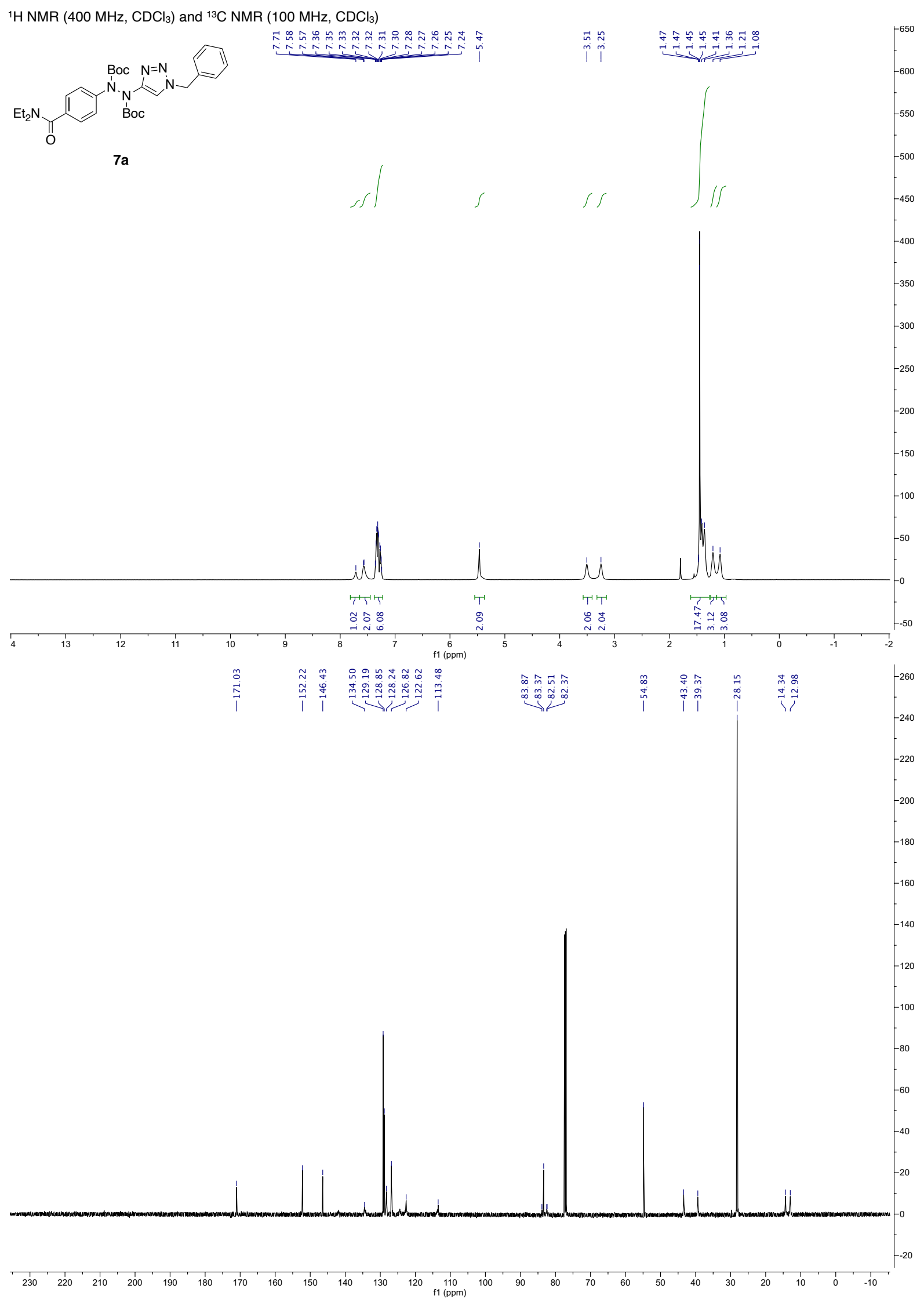




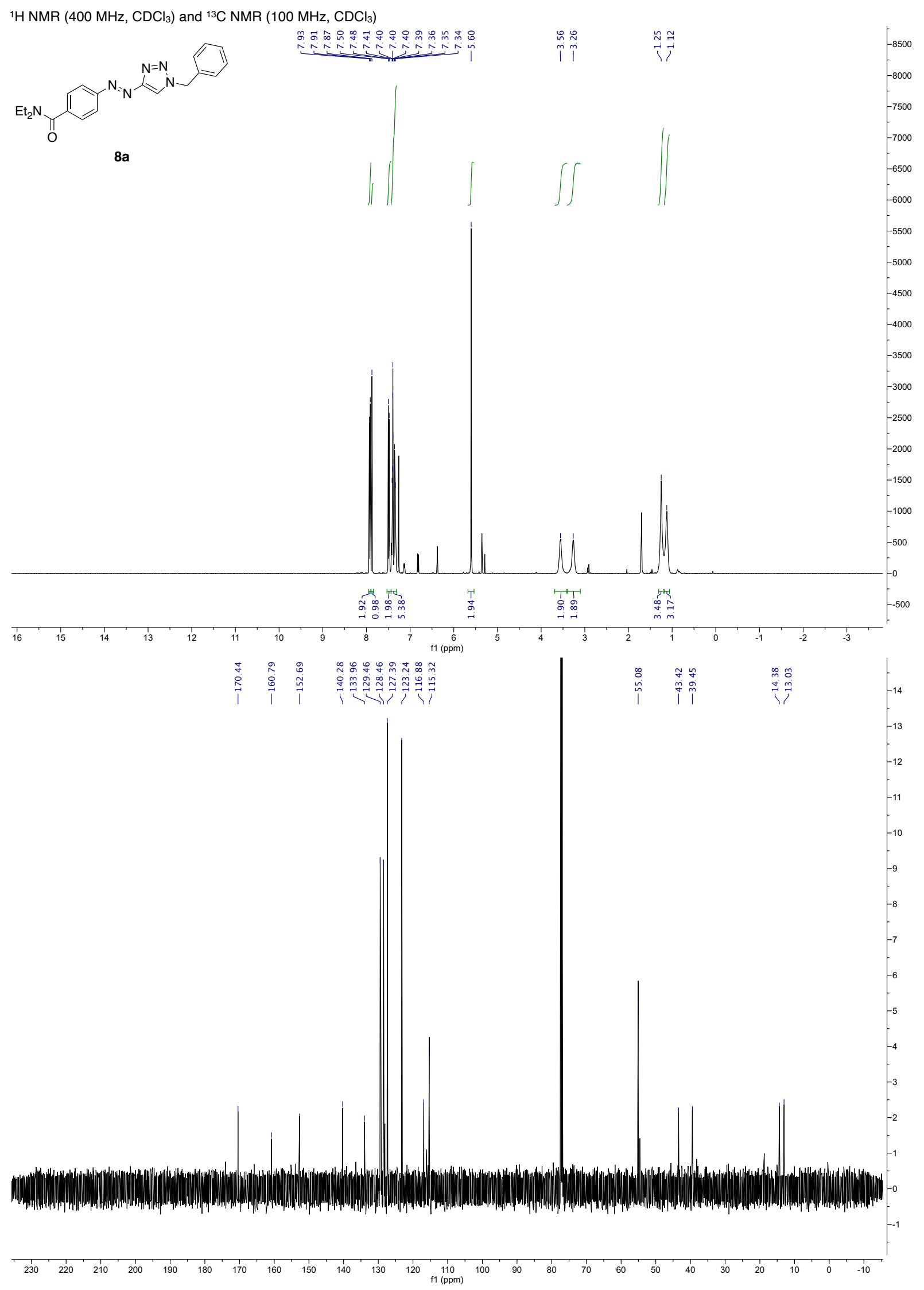




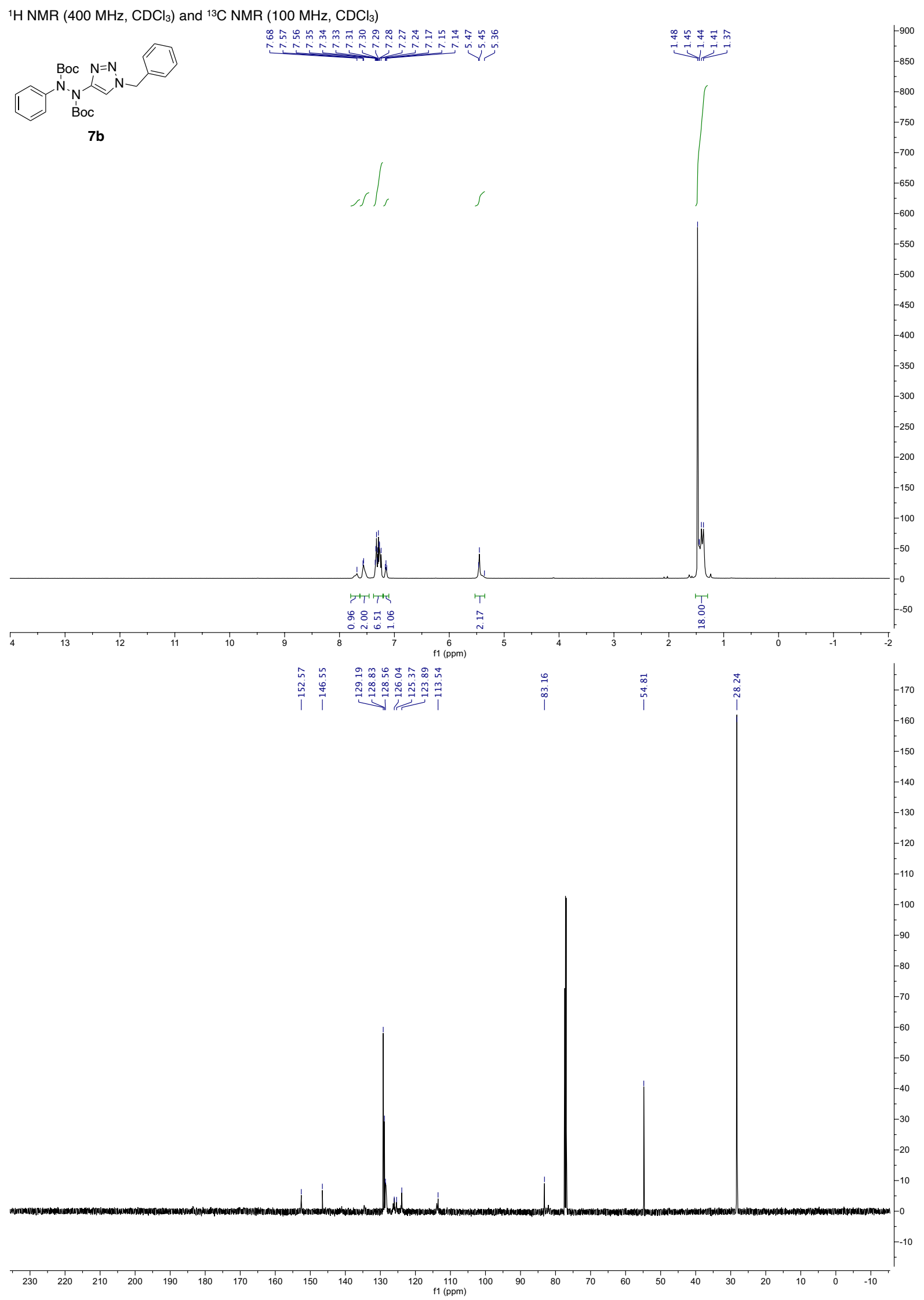




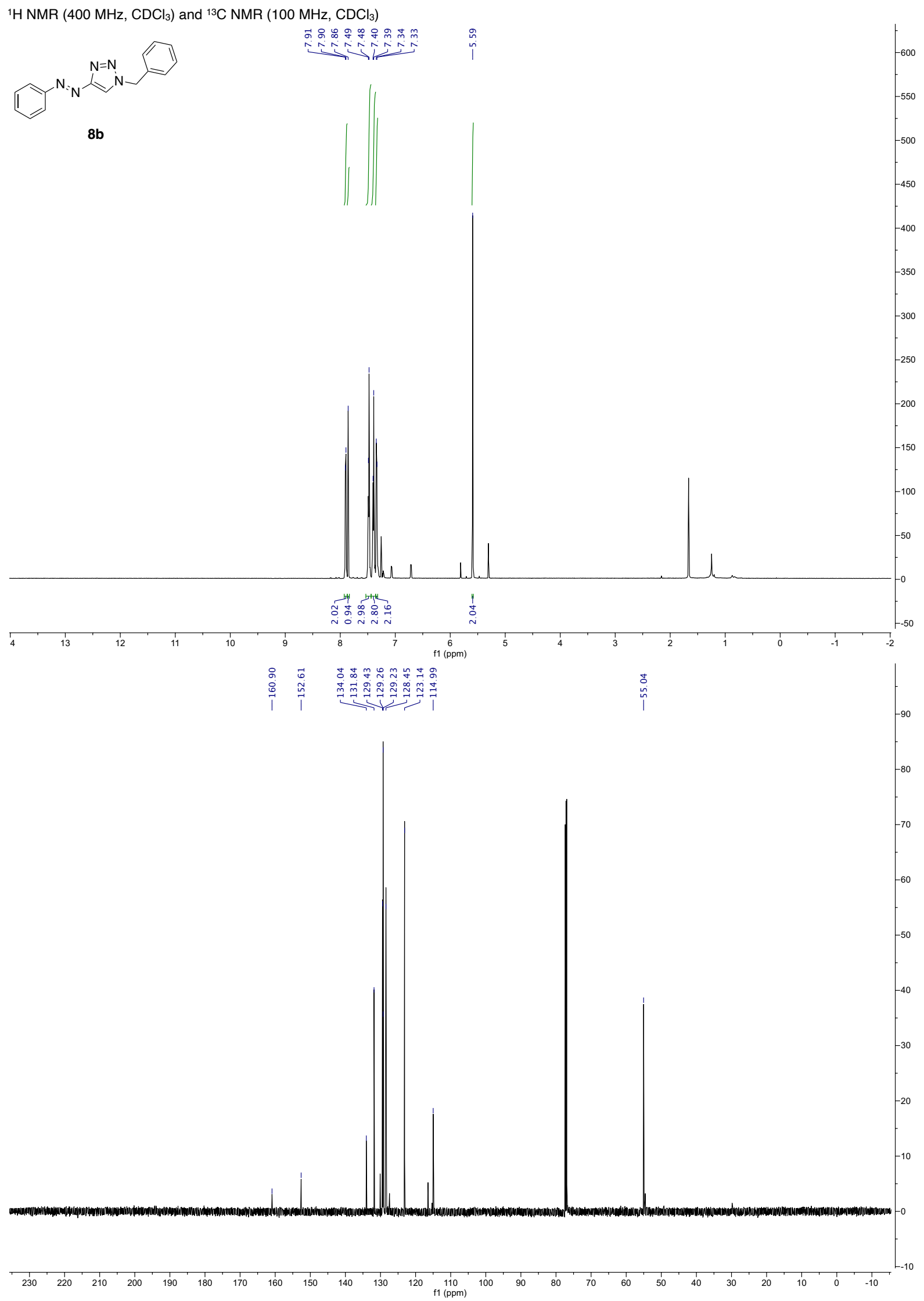


${ }^{1} \mathrm{H}$ NMR (400 MHz, $\left.\mathrm{CDCl}_{3}\right)$ and ${ }^{13} \mathrm{C}$ NMR $\left(100 \mathrm{MHz}, \mathrm{CDCl}_{3}\right)$
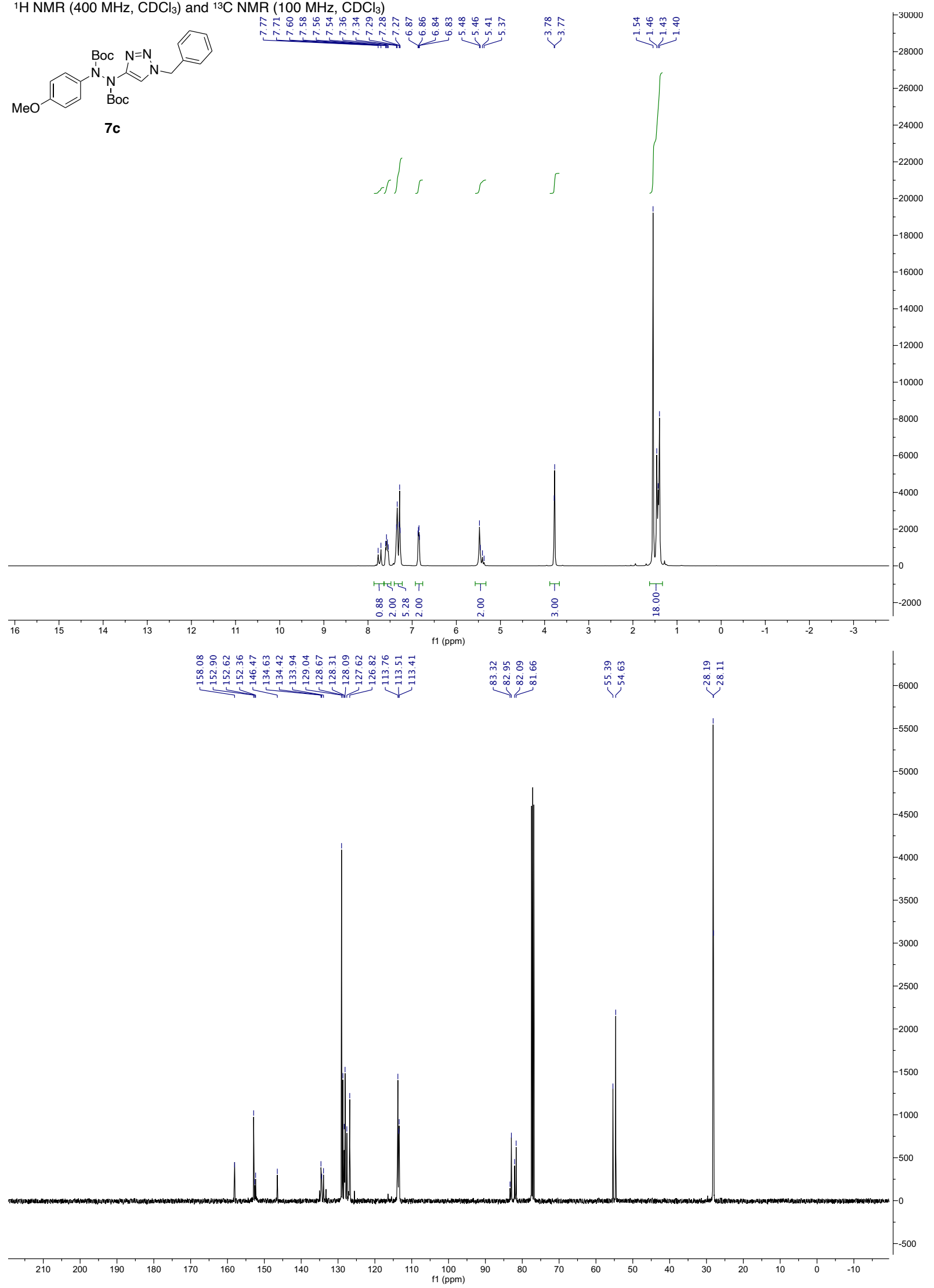


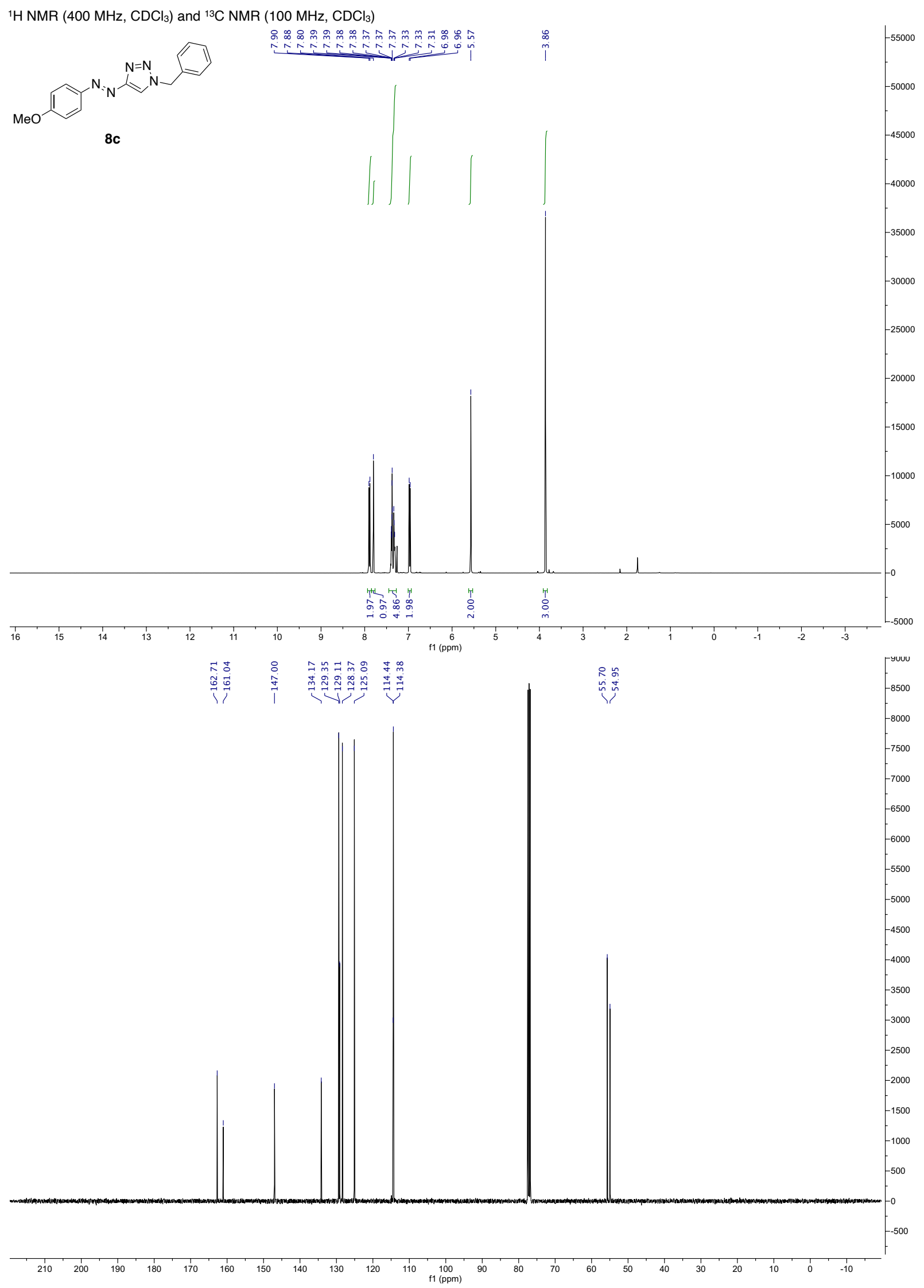


${ }^{1} \mathrm{H}$ NMR (400 MHz, $\mathrm{CDCl}_{3}$ ) and $\left.{ }^{13} \mathrm{C} \mathrm{NMR} \mathrm{(100} \mathrm{MHz,} \mathrm{CDCl}\right)$
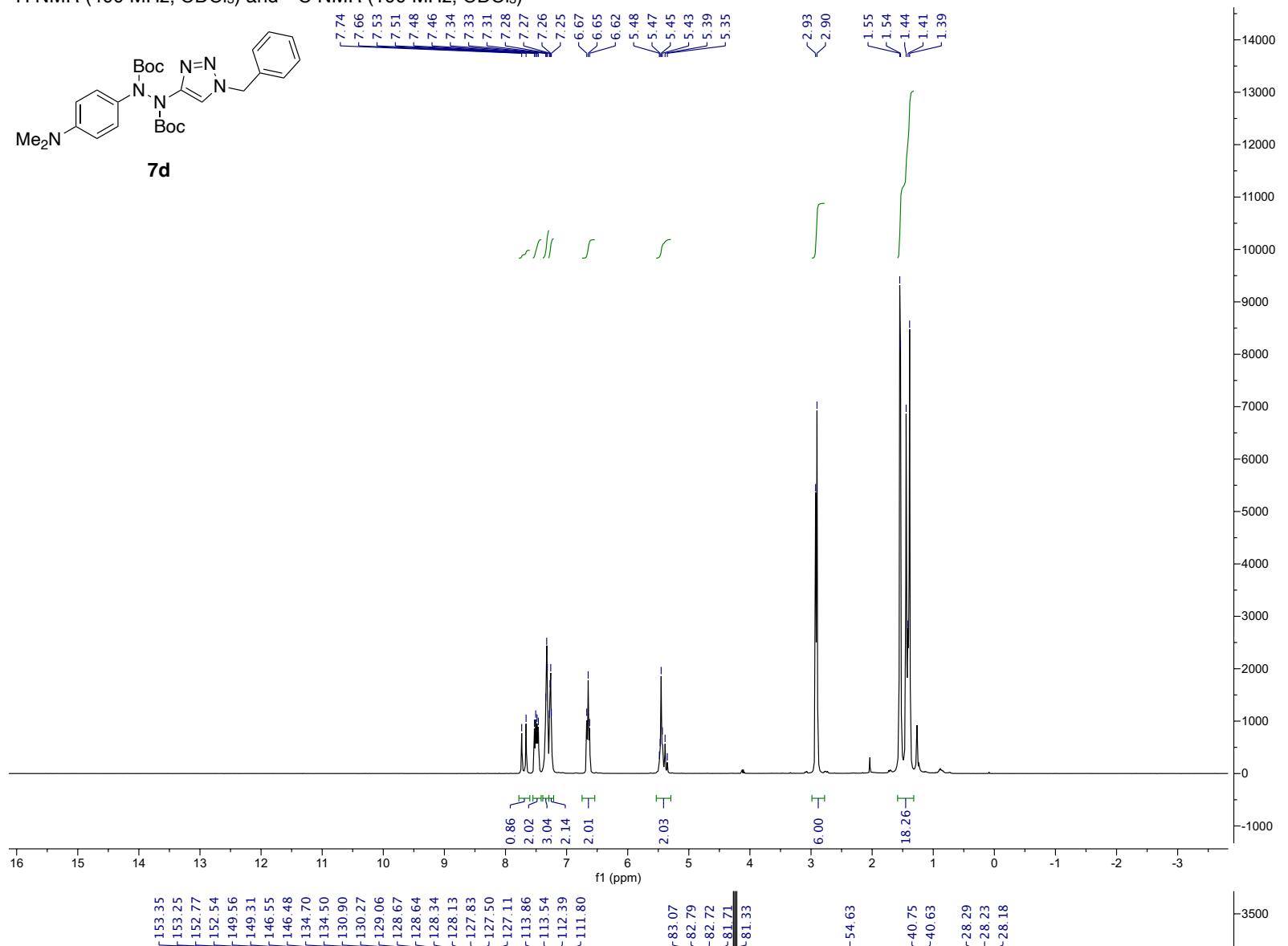

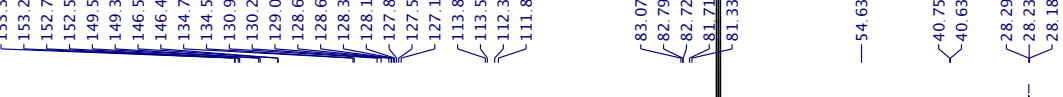

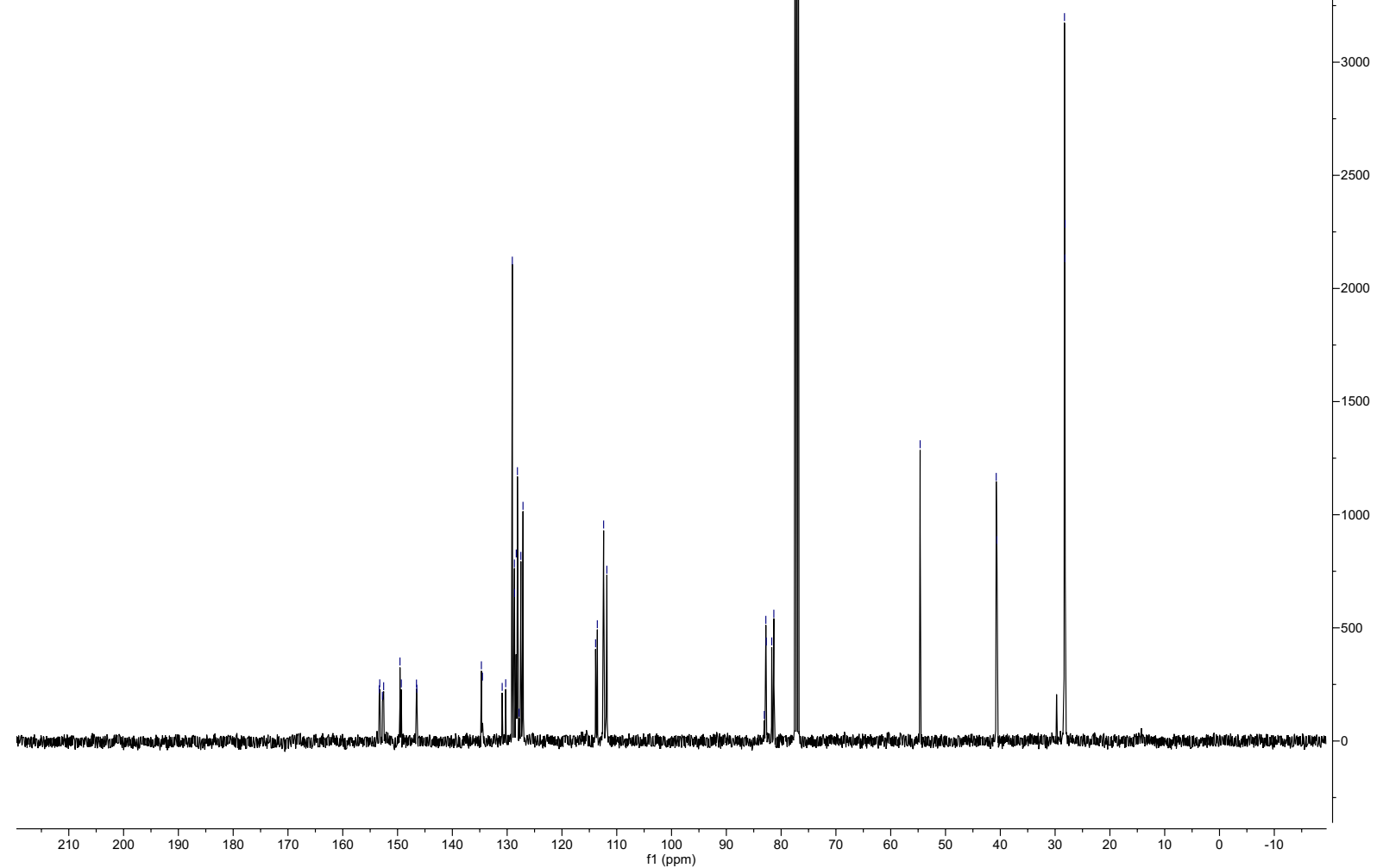


${ }^{1} \mathrm{H}$ NMR (400 MHz, $\left.\mathrm{CDCl}_{3}\right)$ and $\left.{ }^{13} \mathrm{C} \mathrm{NMR} \mathrm{(100} \mathrm{MHz,} \mathrm{CDCl}_{3}\right)$
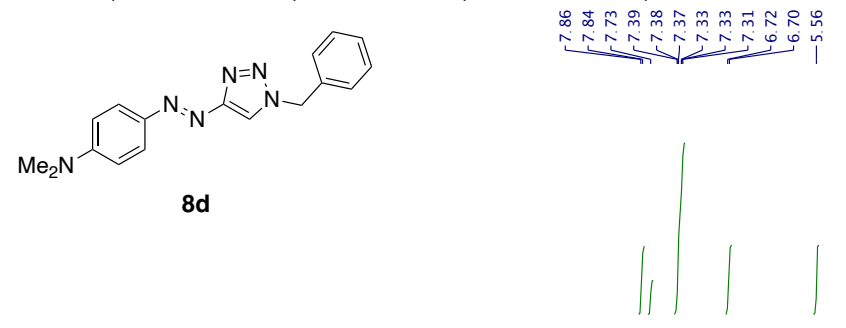

8d

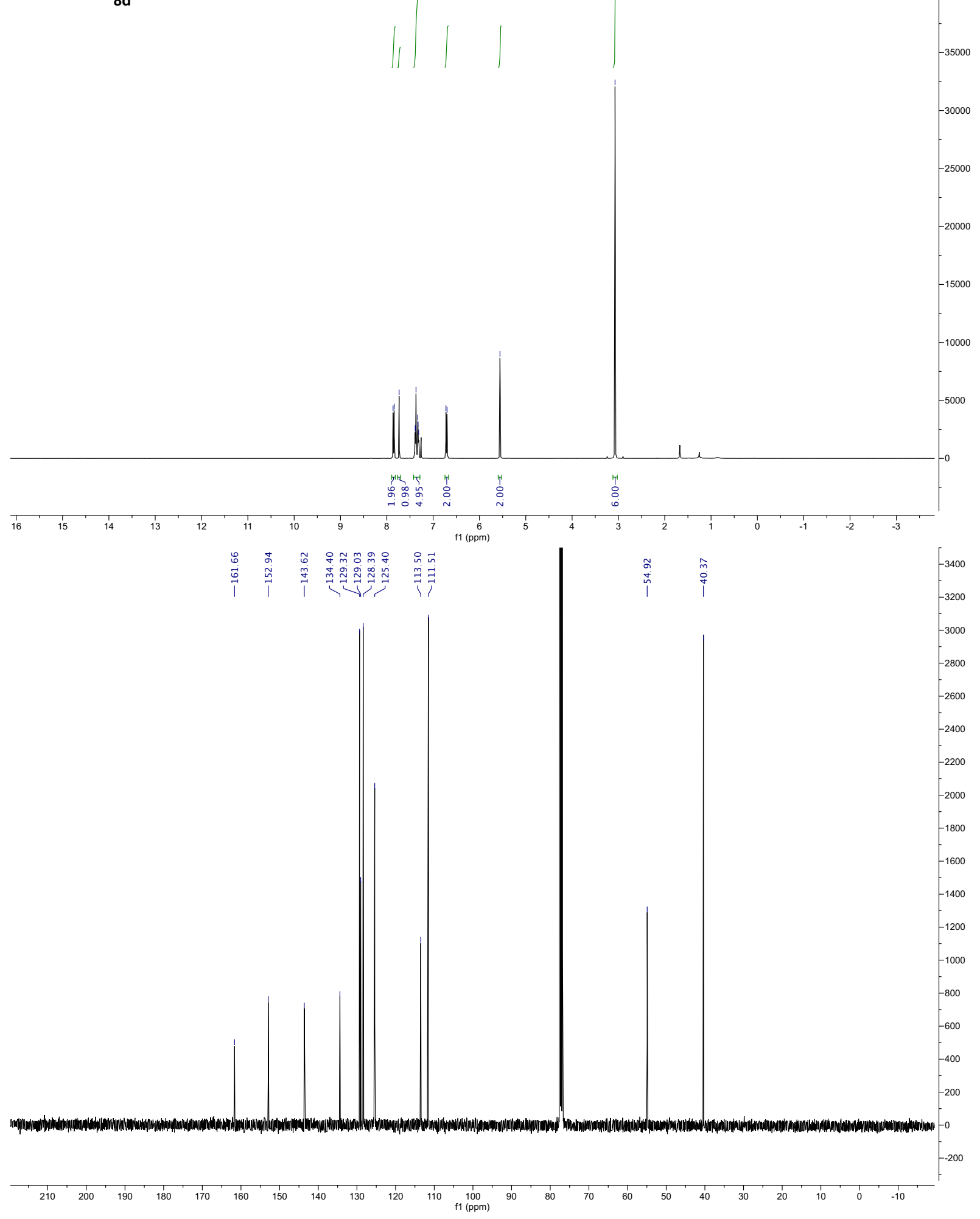


${ }^{1} \mathrm{H}$ NMR $\left(400 \mathrm{MHz}, \mathrm{CDCl}_{3}\right)$ and $\left.{ }^{13} \mathrm{C} \mathrm{NMR} \mathrm{(100} \mathrm{MHz,} \mathrm{CDCl}_{3}\right)$

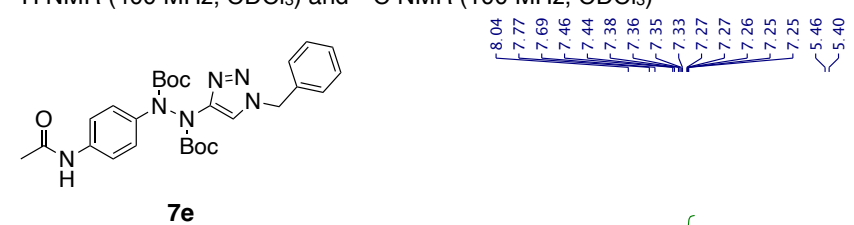

$7 e$

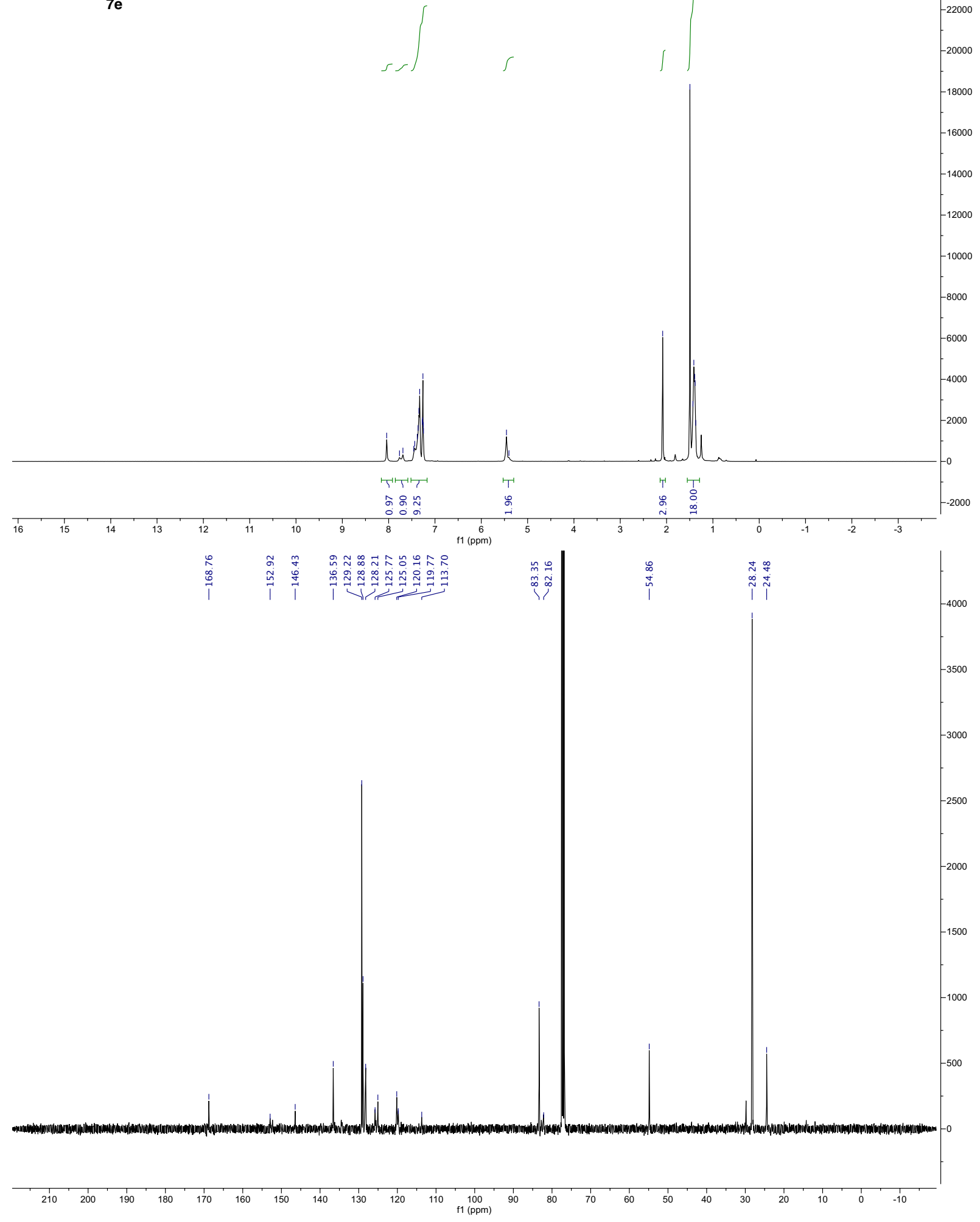




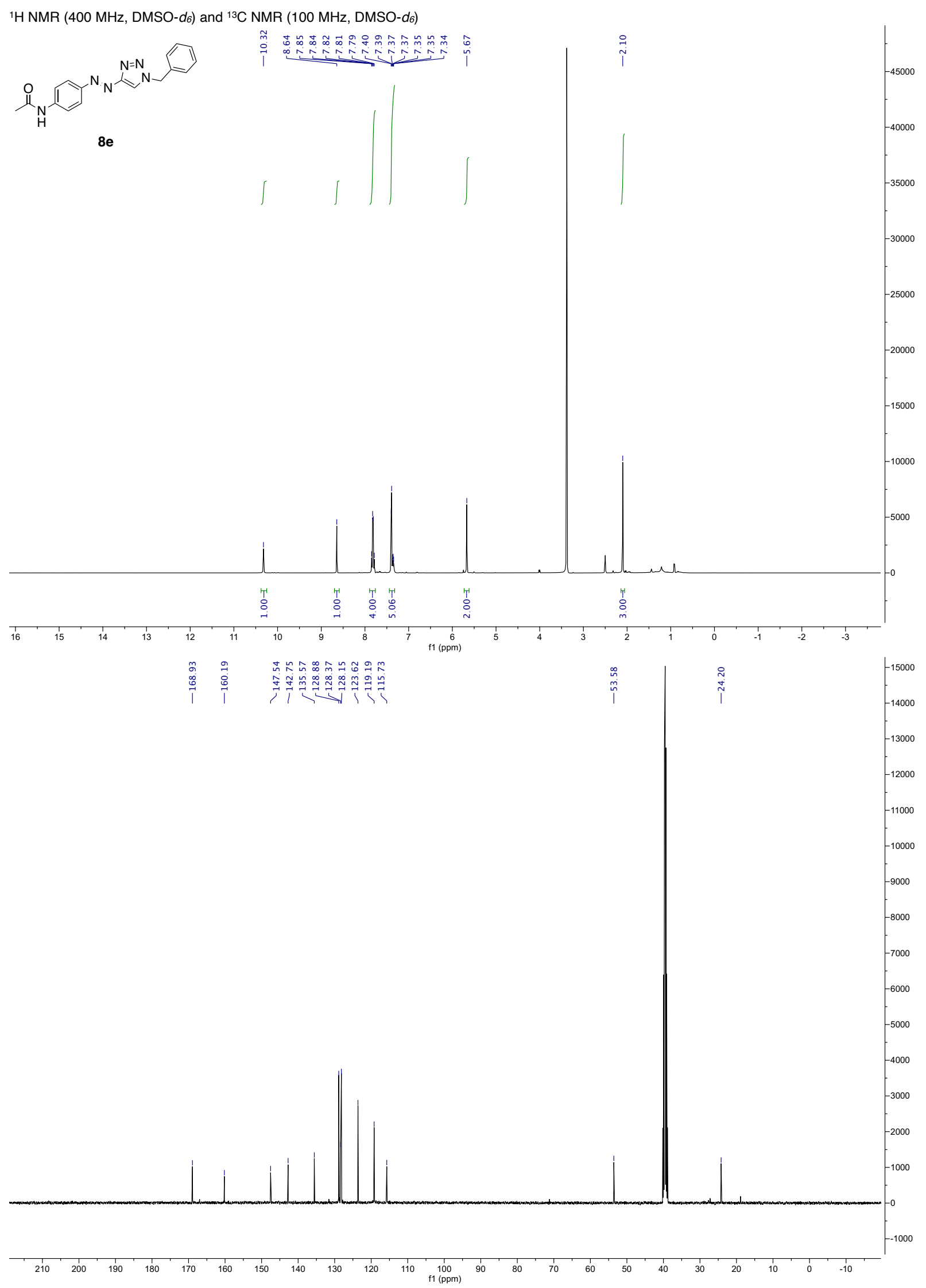


${ }^{1} \mathrm{H}$ NMR (400 MHz, $\mathrm{CDCl}_{3}$ ) and $\left.{ }^{13} \mathrm{C} \mathrm{NMR} \mathrm{(100} \mathrm{MHz,} \mathrm{CDCl}_{3}\right)$
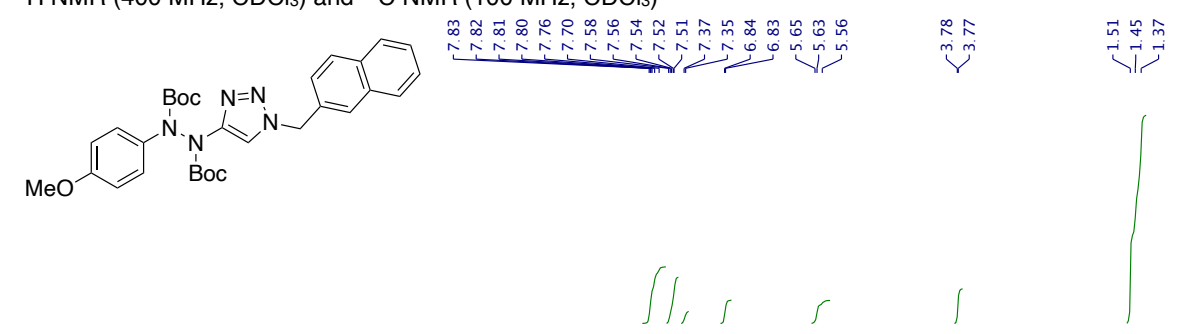

$<40 v 0$
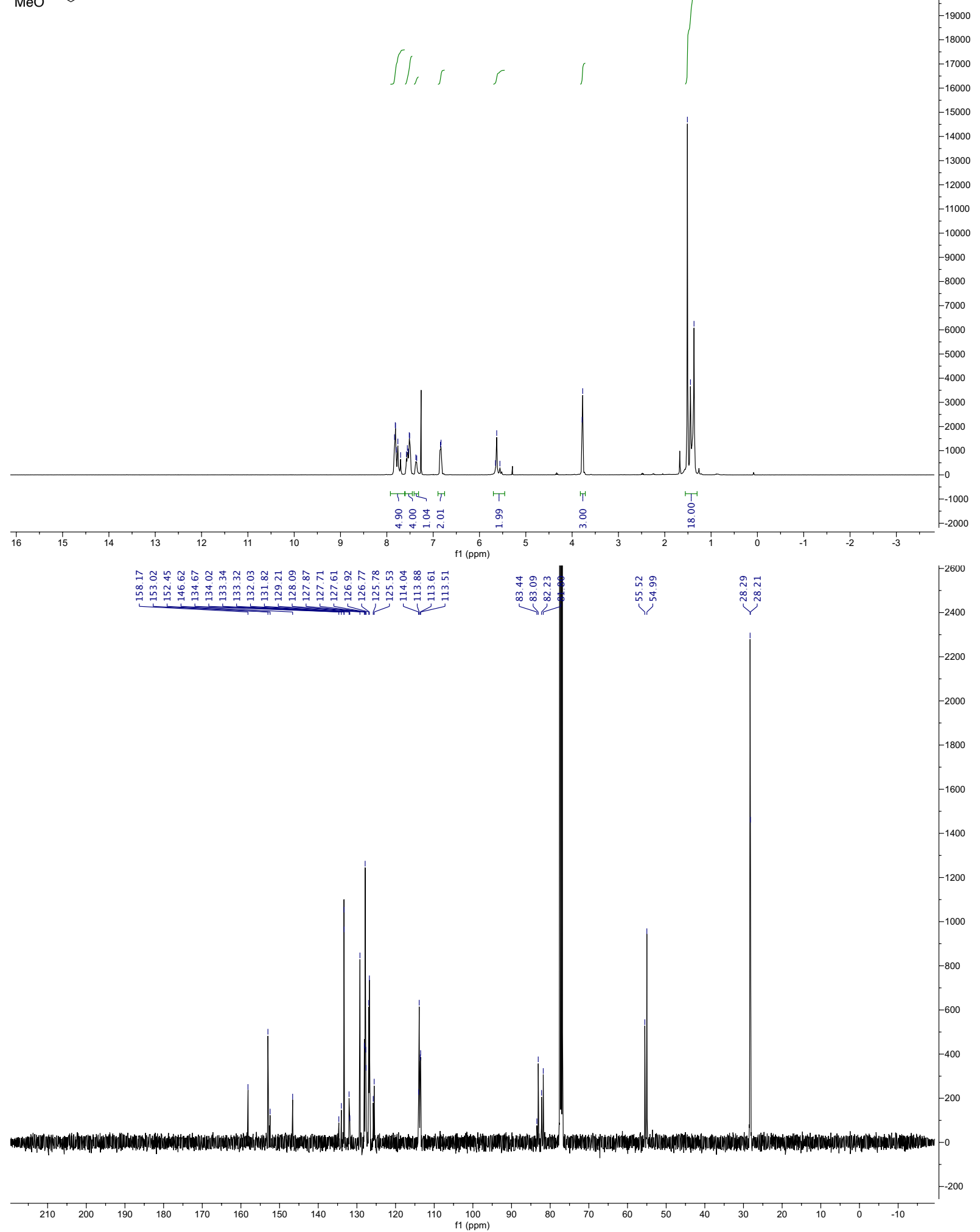


$$
\frac{1}{1}
$$


${ }^{1} \mathrm{H} \mathrm{NMR}\left(400 \mathrm{MHz}, \mathrm{CDCl}_{3}\right)$ and ${ }^{13} \mathrm{C} \mathrm{NMR}\left(100 \mathrm{MHz}, \mathrm{CDCl}_{3}\right)$

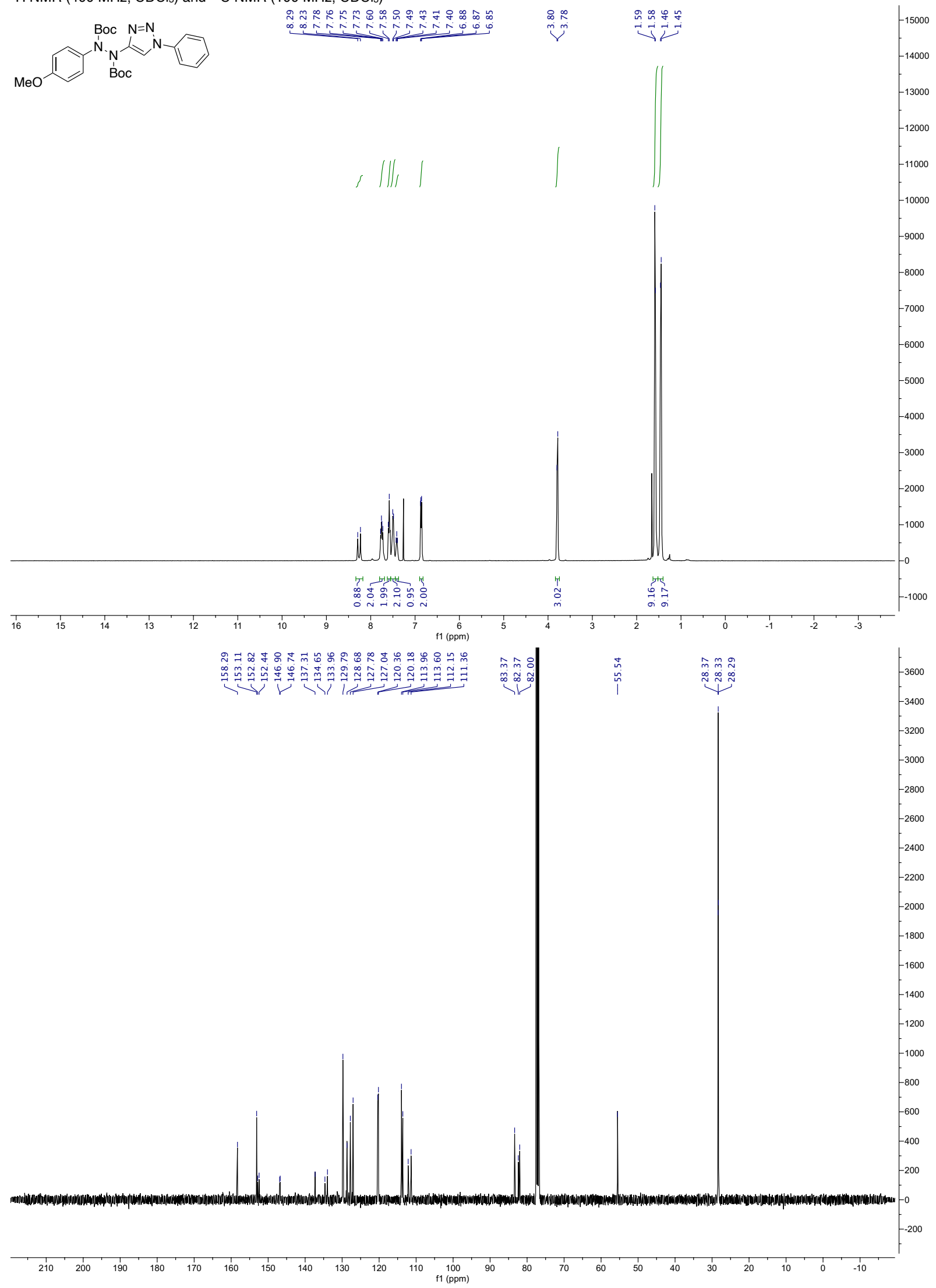


${ }^{1} \mathrm{H} \mathrm{NMR}\left(400 \mathrm{MHz}, \mathrm{CDCl}_{3}\right)$ and ${ }^{13} \mathrm{C} \mathrm{NMR}\left(100 \mathrm{MHz}, \mathrm{CDCl}_{3}\right)$

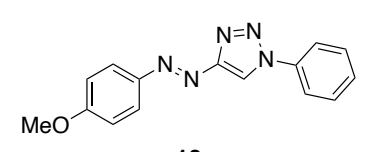

10

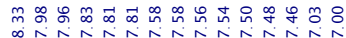

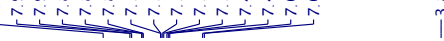
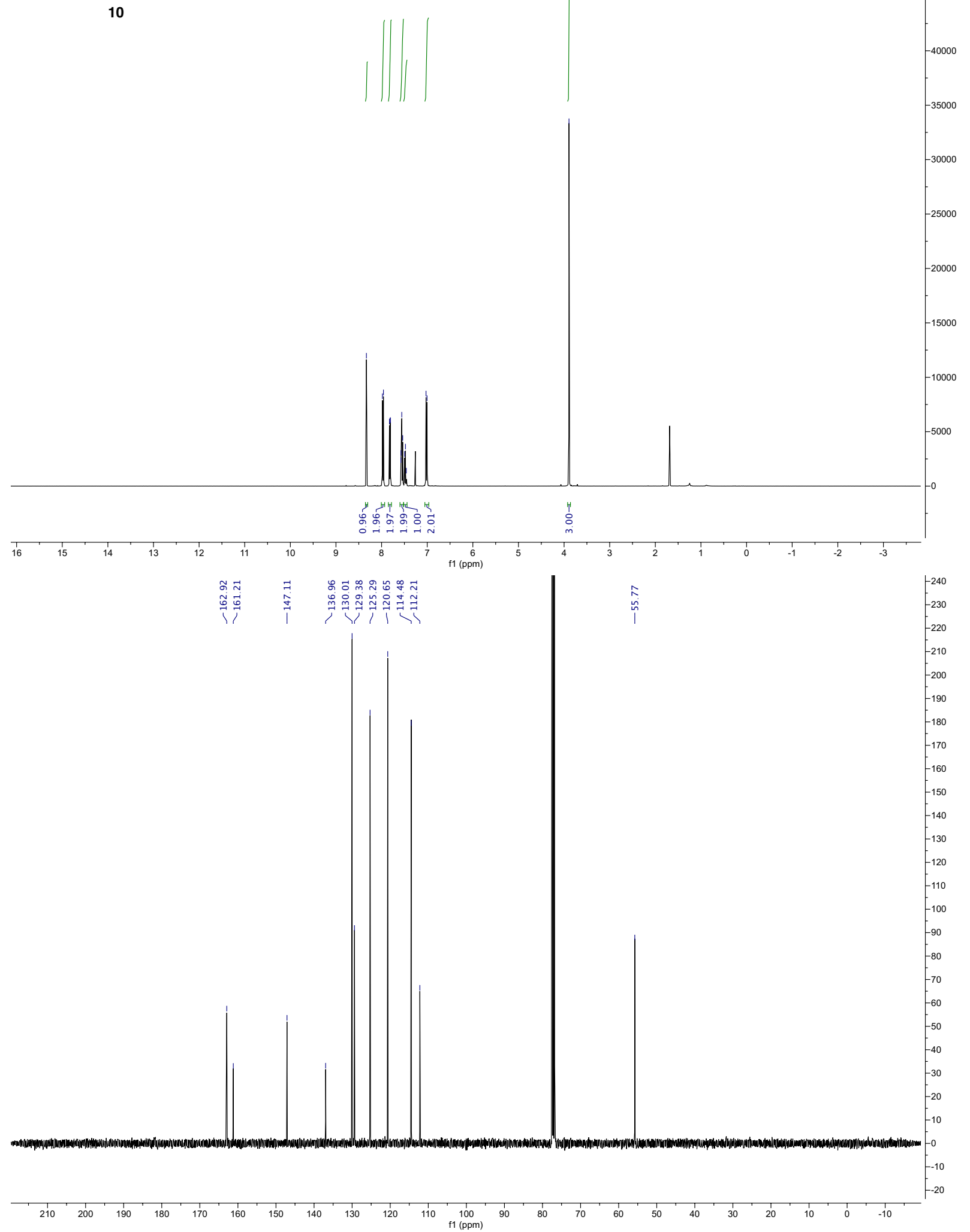
${ }^{1} \mathrm{H}$ NMR $\left(600 \mathrm{MHz}, \mathrm{CDCl}_{3}\right)$ and $\left.{ }^{13} \mathrm{C} \mathrm{NMR} \mathrm{(150} \mathrm{MHz,} \mathrm{CDCl}_{3}\right)$

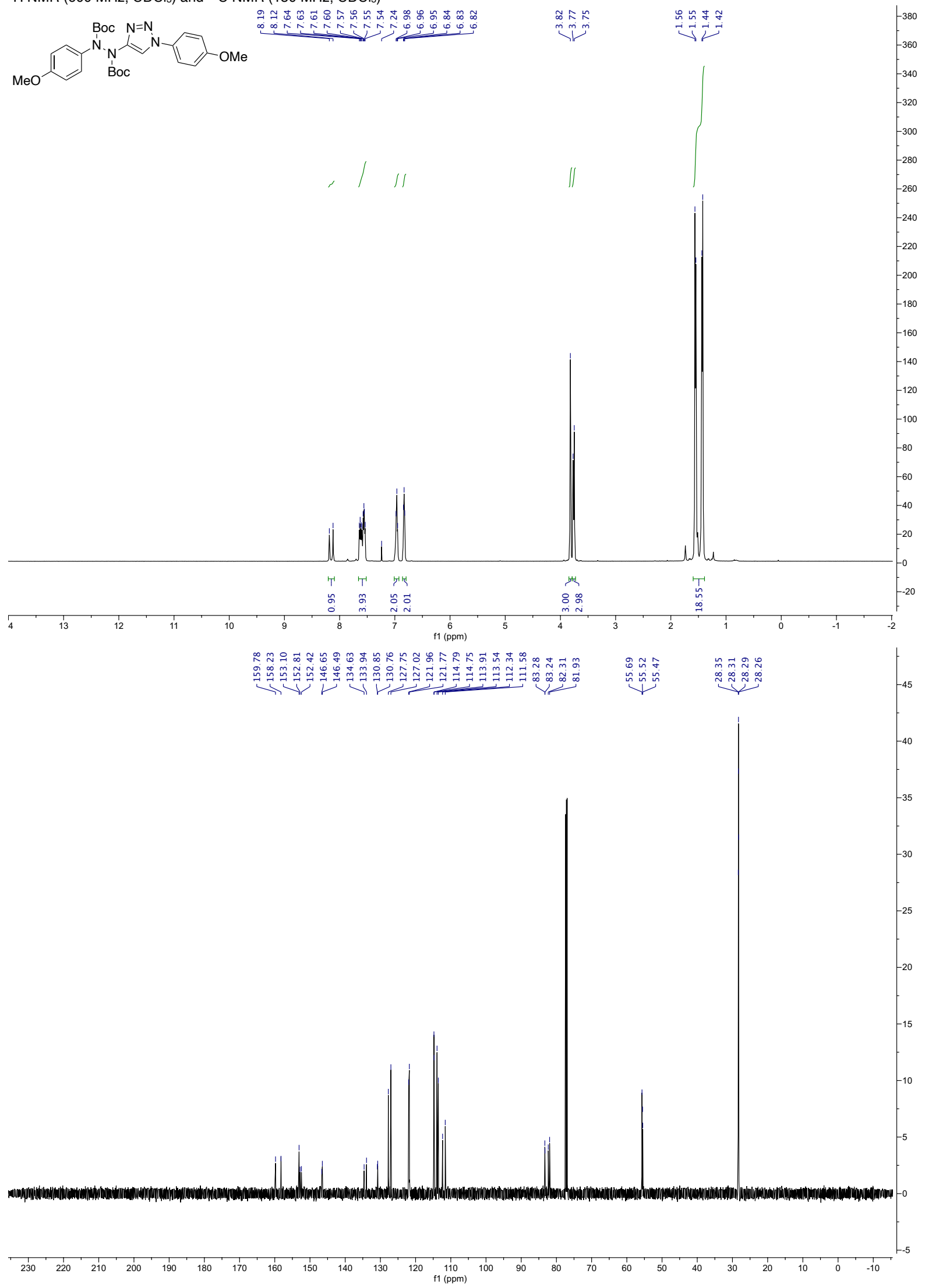


${ }^{1} \mathrm{H} \mathrm{NMR}\left(600 \mathrm{MHz}, \mathrm{CDCl}_{3}\right)$ and ${ }^{13} \mathrm{C} \mathrm{NMR}\left(150 \mathrm{MHz}, \mathrm{CDCl}_{3}\right)$

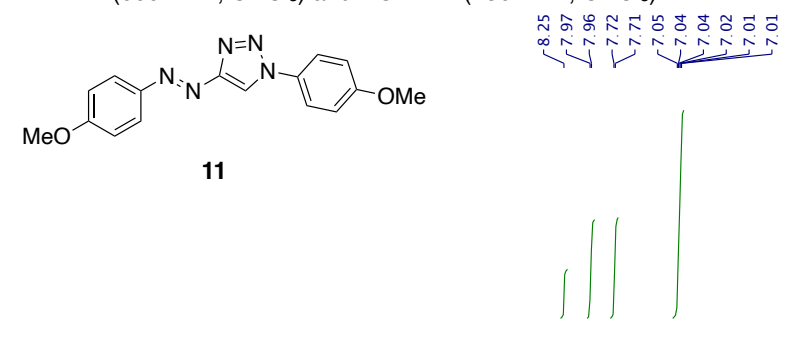

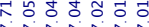

$\underbrace{\infty}_{\substack{\infty \\ \infty}}$

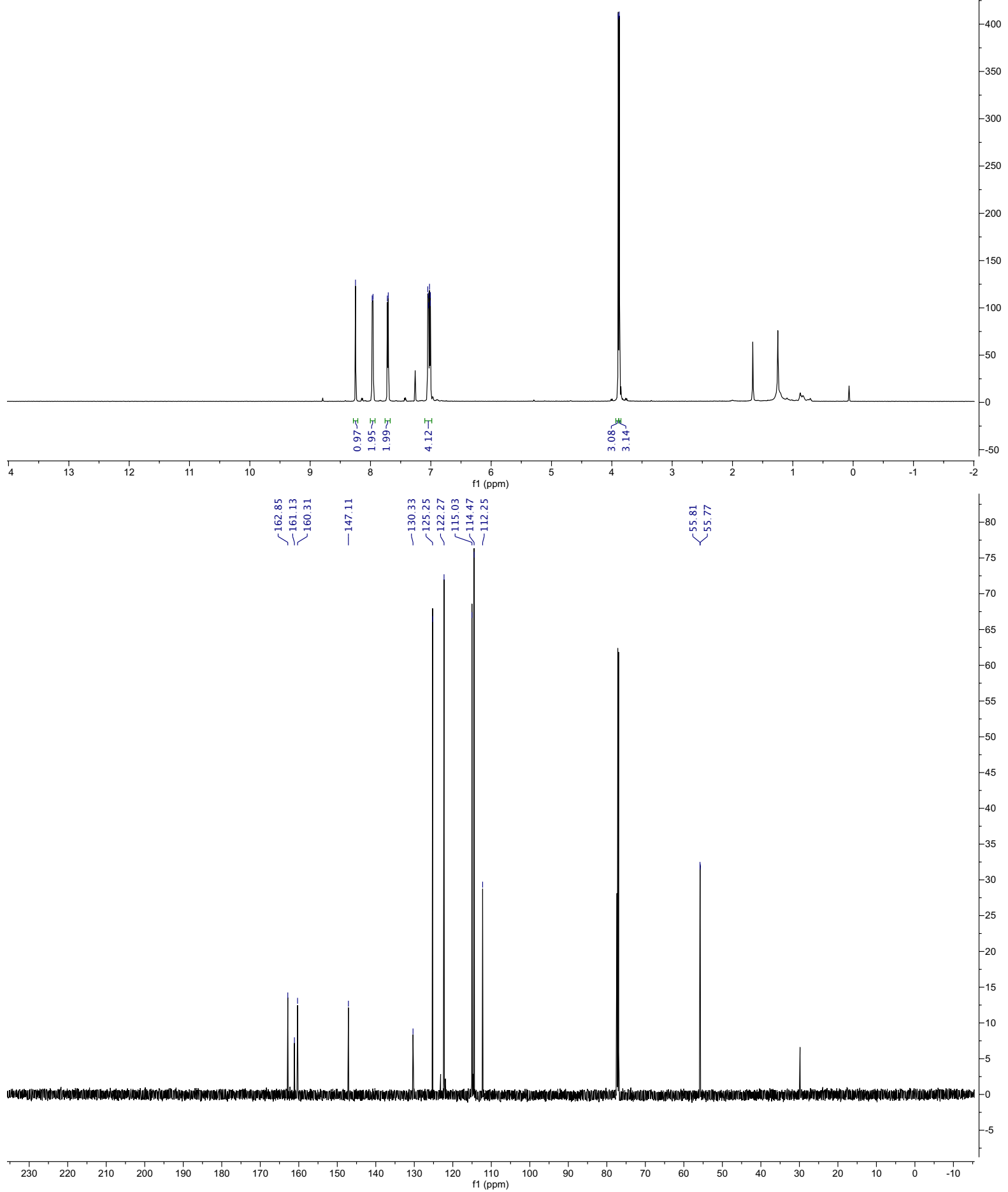


${ }^{1} \mathrm{H} \mathrm{NMR}\left(400 \mathrm{MHz}, \mathrm{CDCl}_{3}\right)$ and ${ }^{13} \mathrm{C} \mathrm{NMR}\left(100 \mathrm{MHz}, \mathrm{CDCl}_{3}\right)$
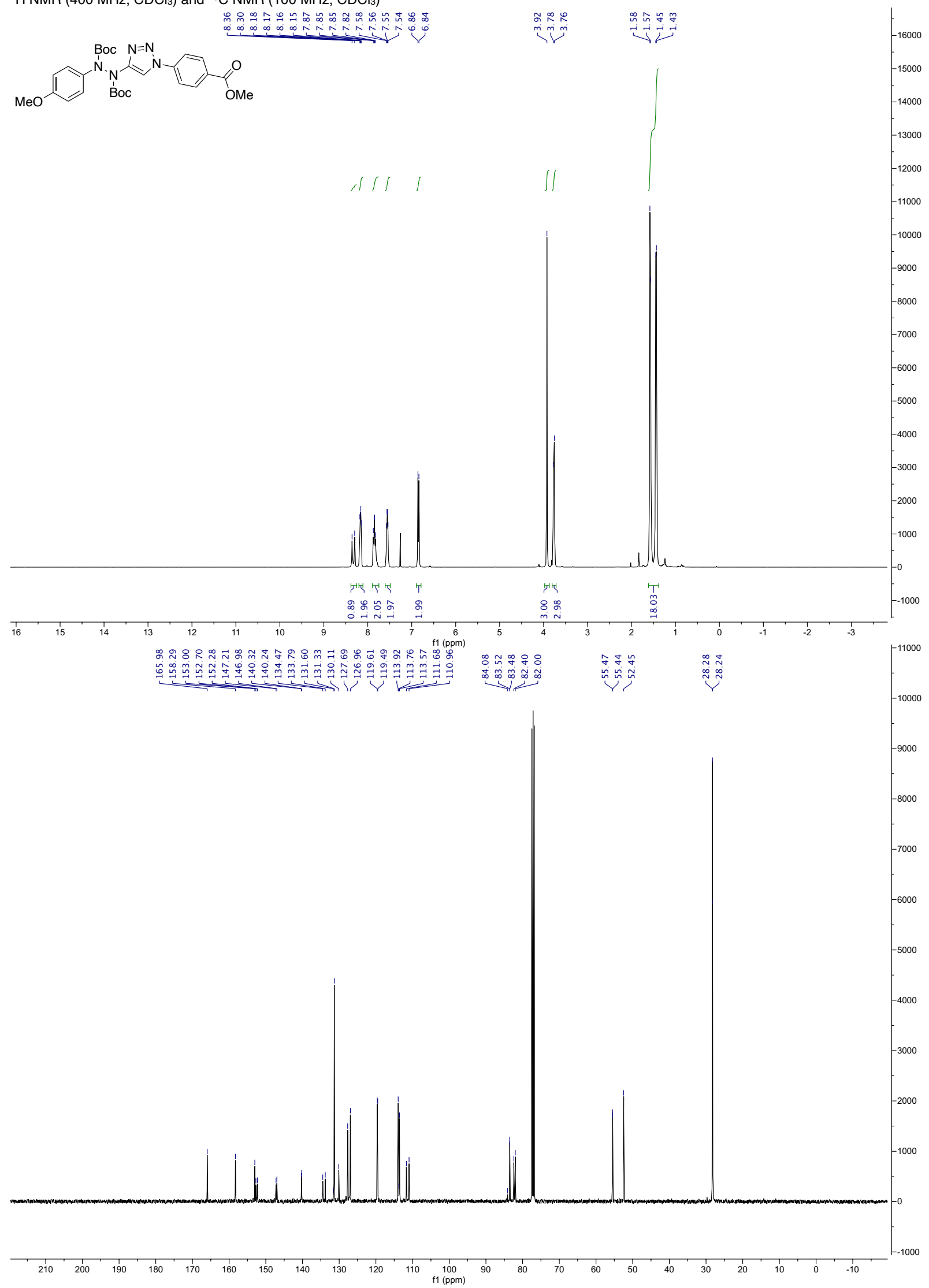
${ }^{1} \mathrm{H}$ NMR (400 MHz, $\mathrm{CDCl}_{3}$ ) and $\left.{ }^{13} \mathrm{C} \mathrm{NMR} \mathrm{(100} \mathrm{MHz,} \mathrm{CDCl}_{3}\right)$

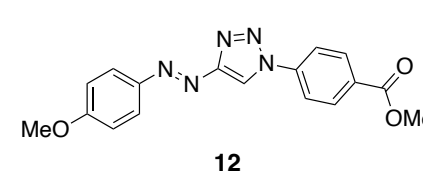

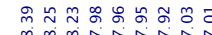

ì

$-22000$

21000

20000

12

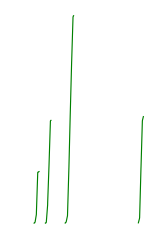

19000

$-18000$

$-17000$

$-16000$

$-15000$

$-14000$

$-13000$

$-12000$

$-11000$

$-10000$

$-9000$

$-8000$

$-7000$

$-6000$

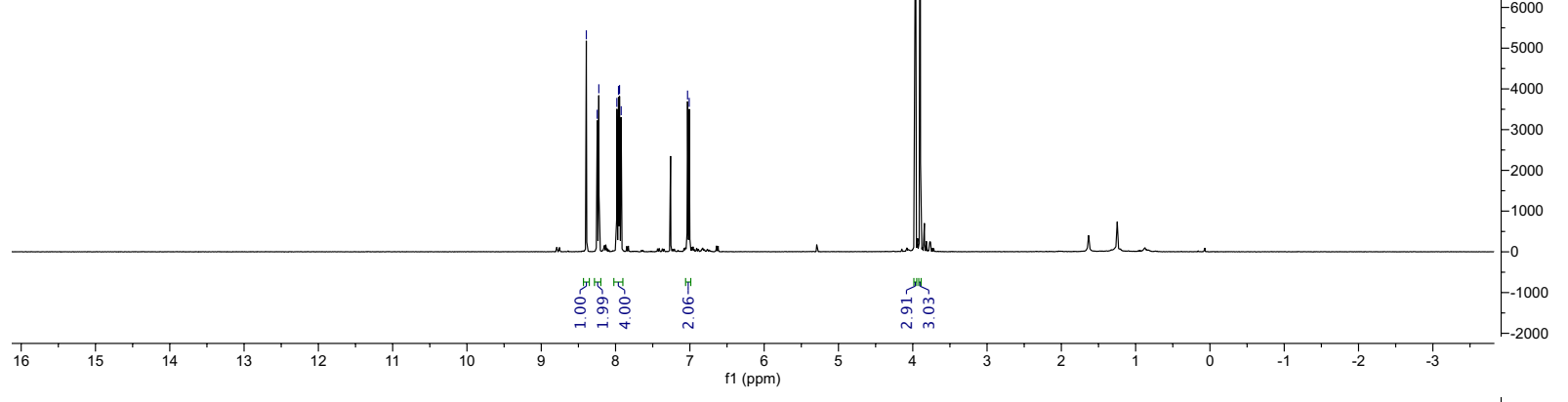

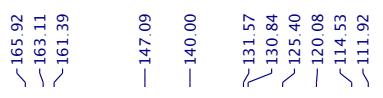

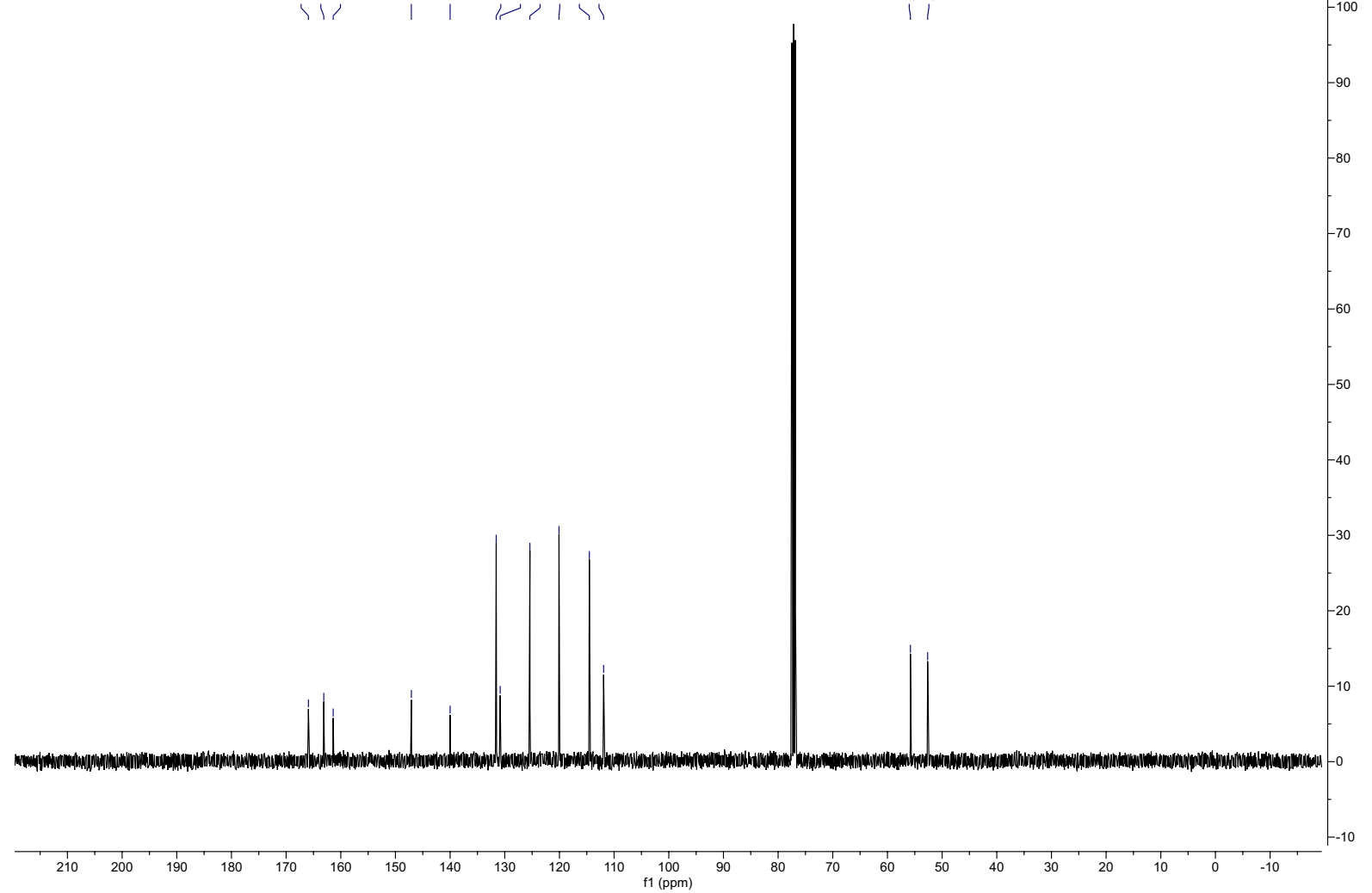


${ }^{1} \mathrm{H}$ NMR $\left(600 \mathrm{MHz}\right.$, DMSO- $d_{6}$ ) and ${ }^{13} \mathrm{C}$ NMR (150 MHz, DMSO- $\left.d_{6}\right)$

ill

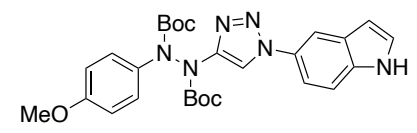

, th, I,

ur uidid d

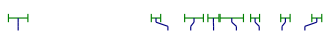

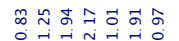

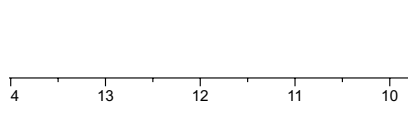

望

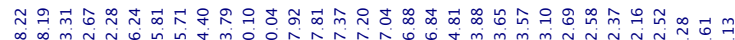

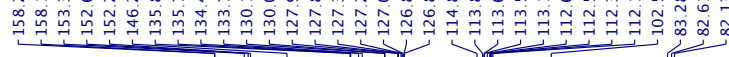

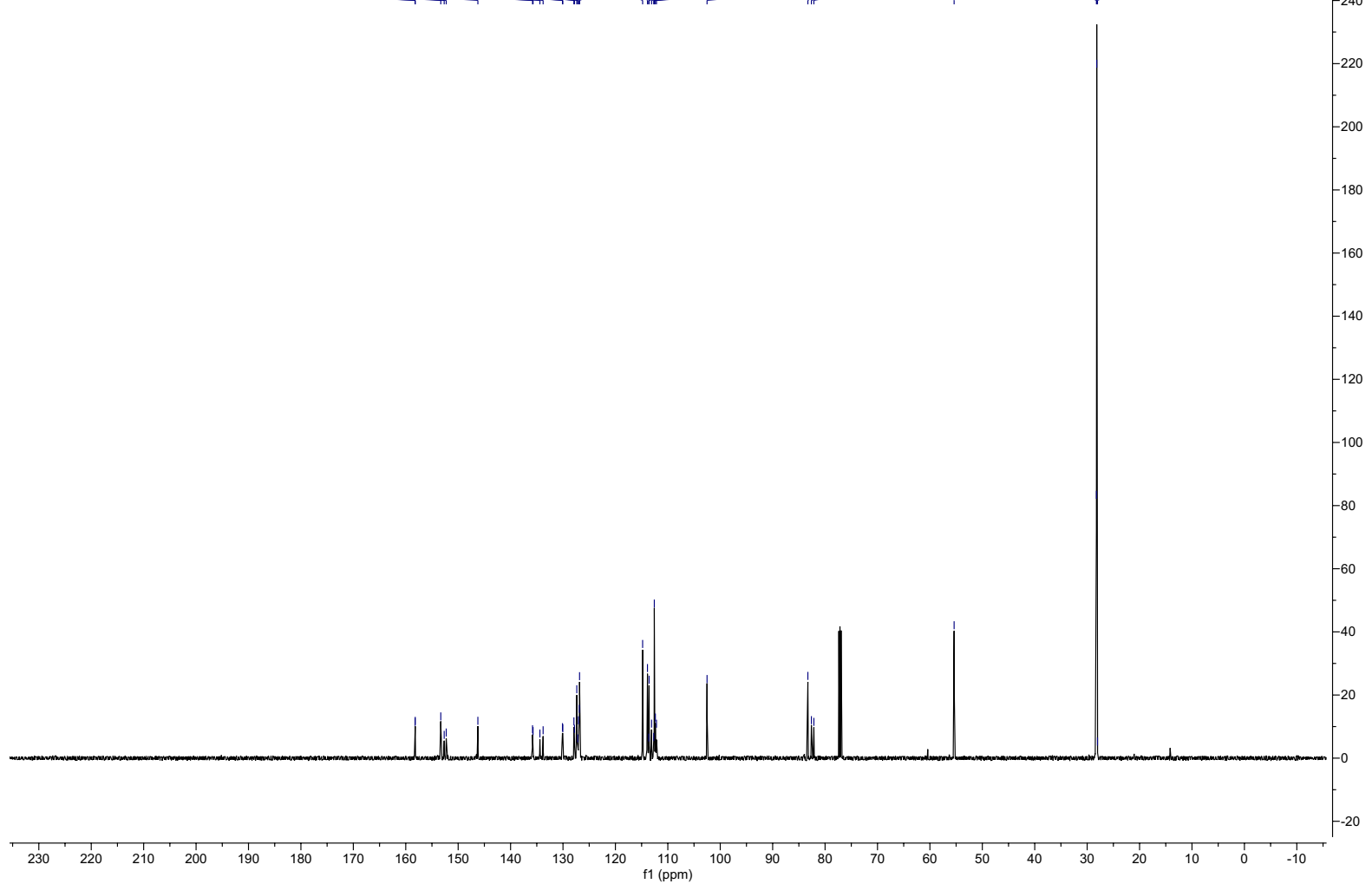


${ }^{1} \mathrm{H}$ NMR $\left(600 \mathrm{MHz}\right.$, DMSO- $\left.d_{6}\right)$ and ${ }^{13} \mathrm{C}$ NMR $\left(150 \mathrm{MHz}\right.$, DMSO- $\left.d_{6}\right)$

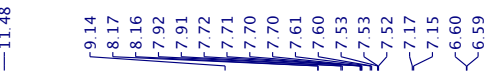

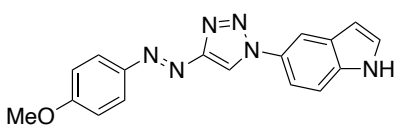

13
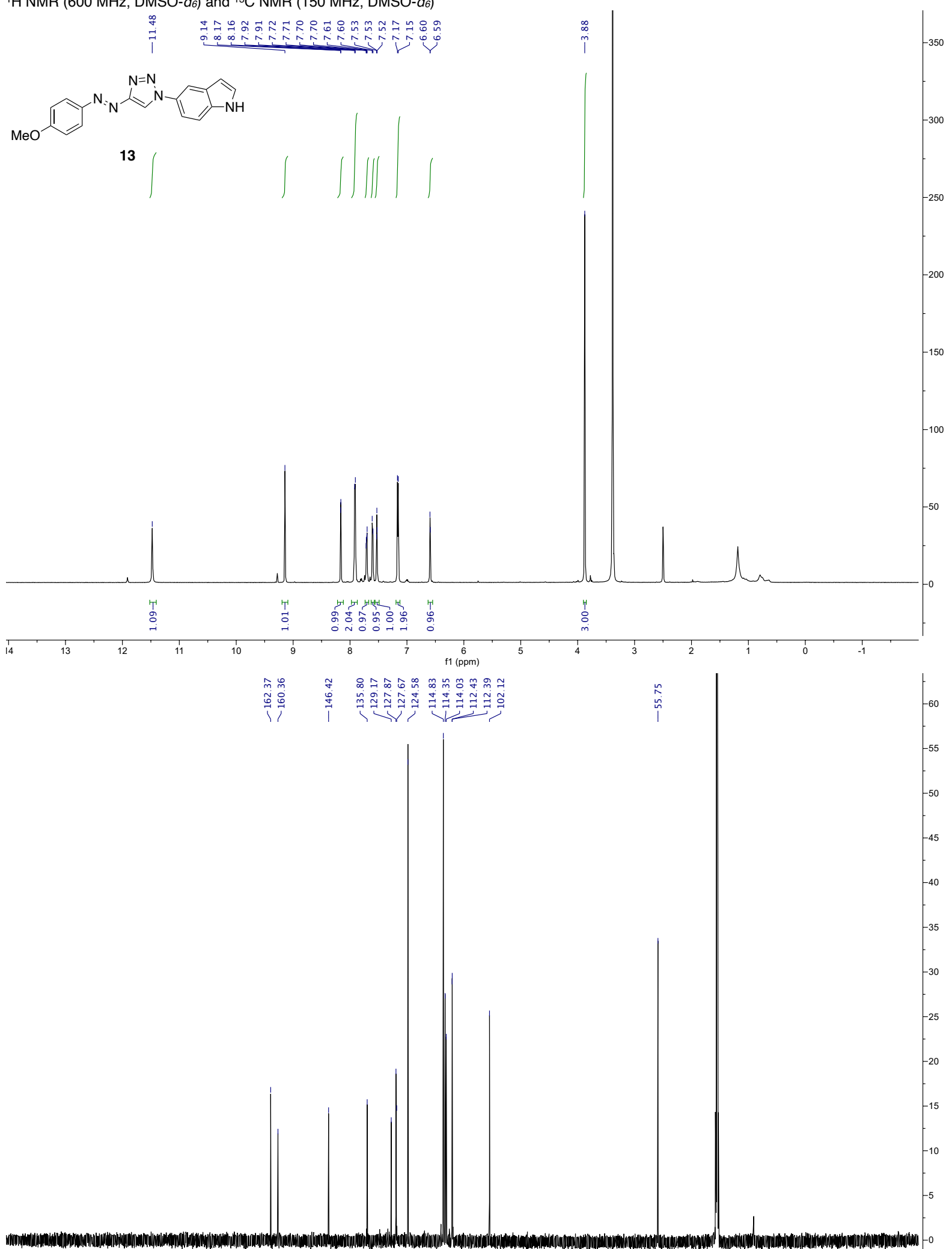

ram

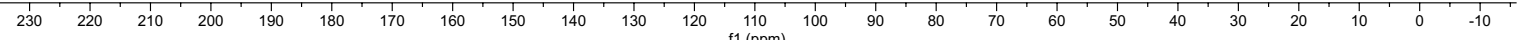


${ }^{1} \mathrm{H} \mathrm{NMR}\left(400 \mathrm{MHz}, \mathrm{CDCl}_{3}\right)$ and ${ }^{13} \mathrm{C} \mathrm{NMR}\left(100 \mathrm{MHz}, \mathrm{CDCl}_{3}\right)$

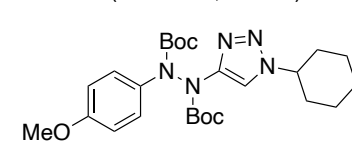

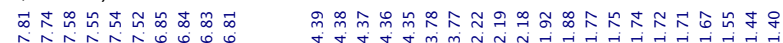

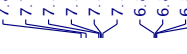

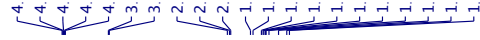

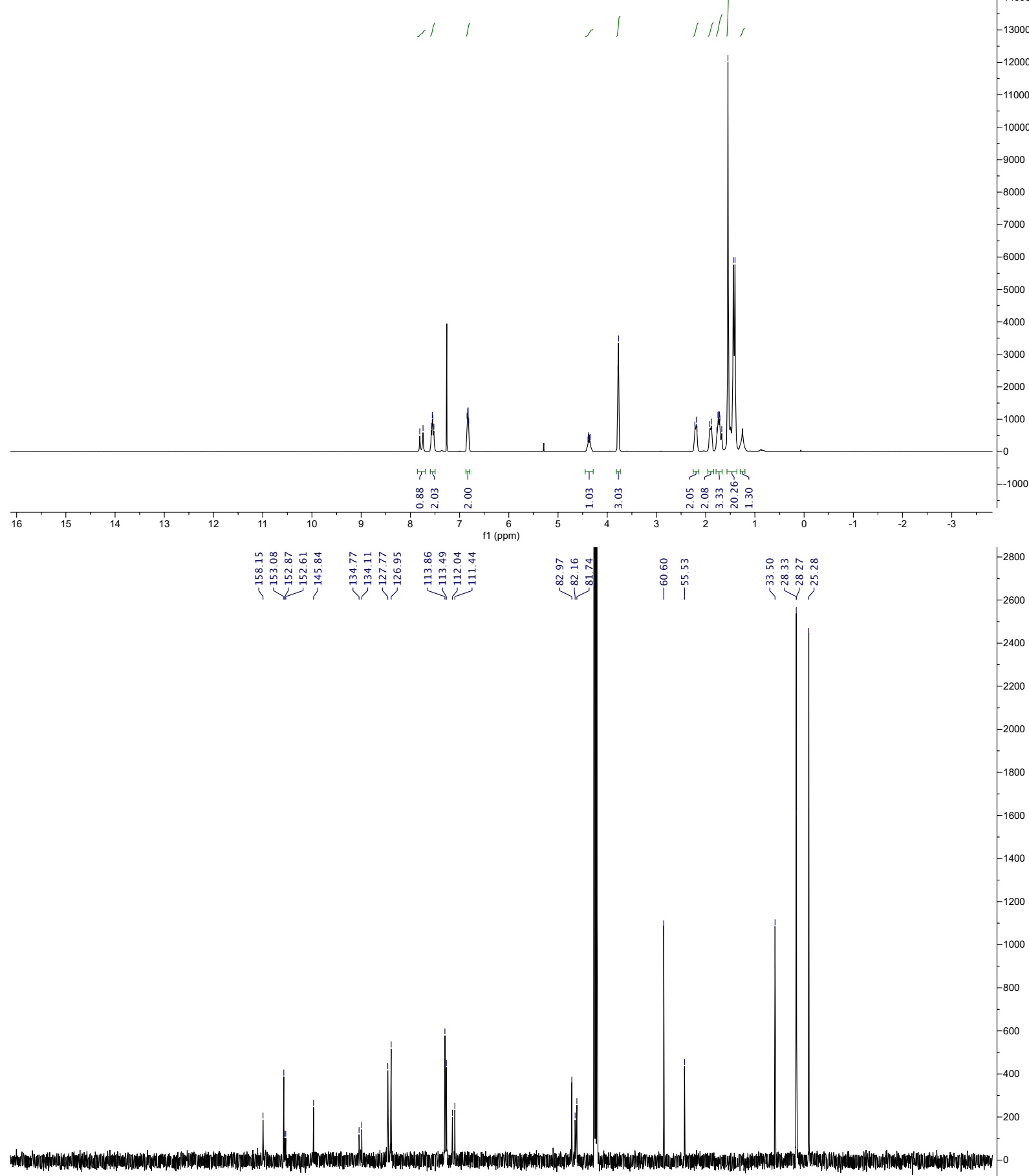

13000
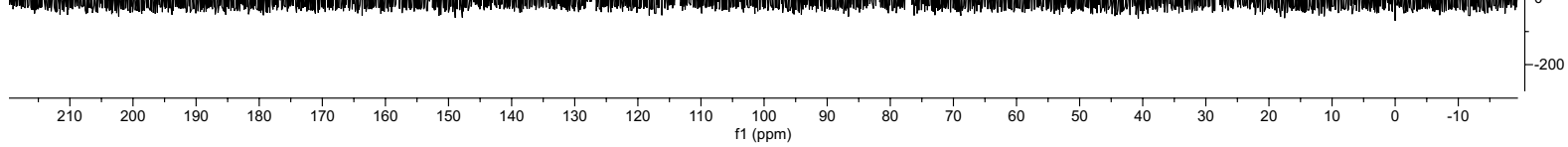
${ }^{1} \mathrm{H}$ NMR (400 MHz, $\left.\mathrm{CDCl}_{3}\right)$ and $\left.{ }^{13} \mathrm{C} \mathrm{NMR} \mathrm{(100} \mathrm{MHz,} \mathrm{CDCl}_{3}\right)$

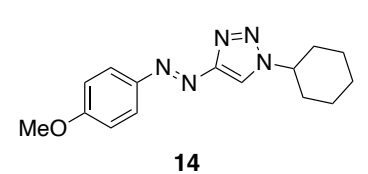

14

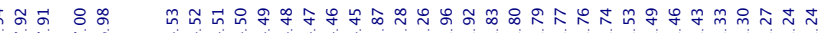

证

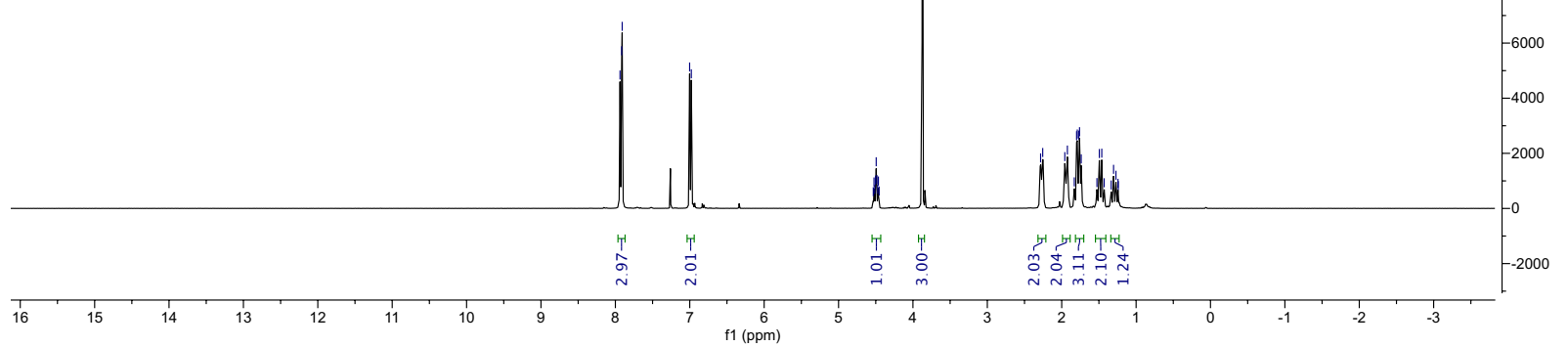

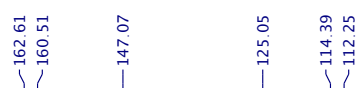

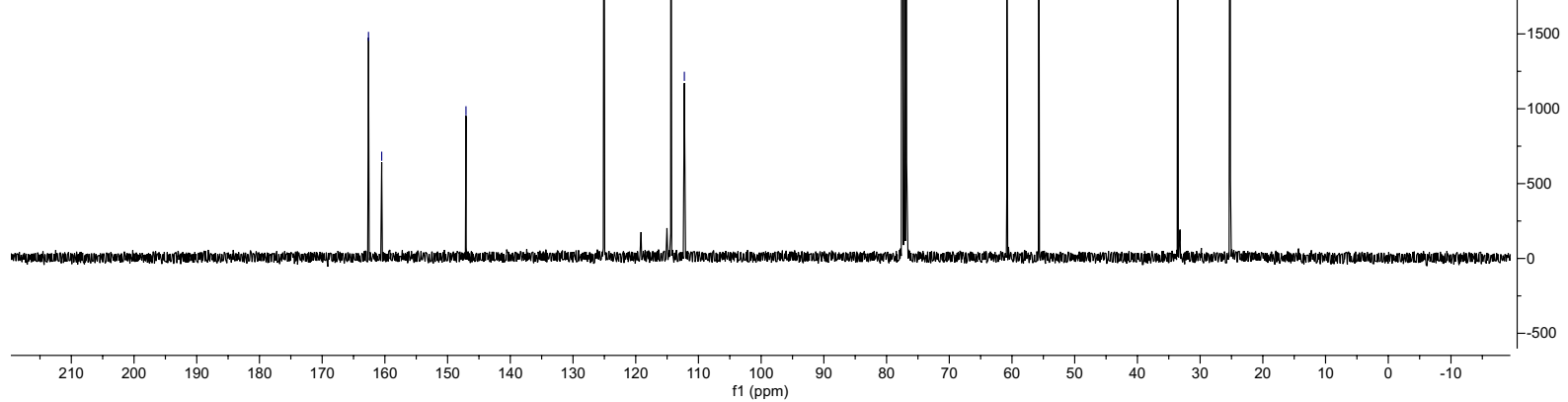


${ }^{1} \mathrm{H}$ NMR (400 MHz, $\left.\mathrm{CDCl}_{3}\right)$ and $\left.{ }^{13} \mathrm{C} \mathrm{NMR} \mathrm{(100} \mathrm{MHz,} \mathrm{CDCl}_{3}\right)$

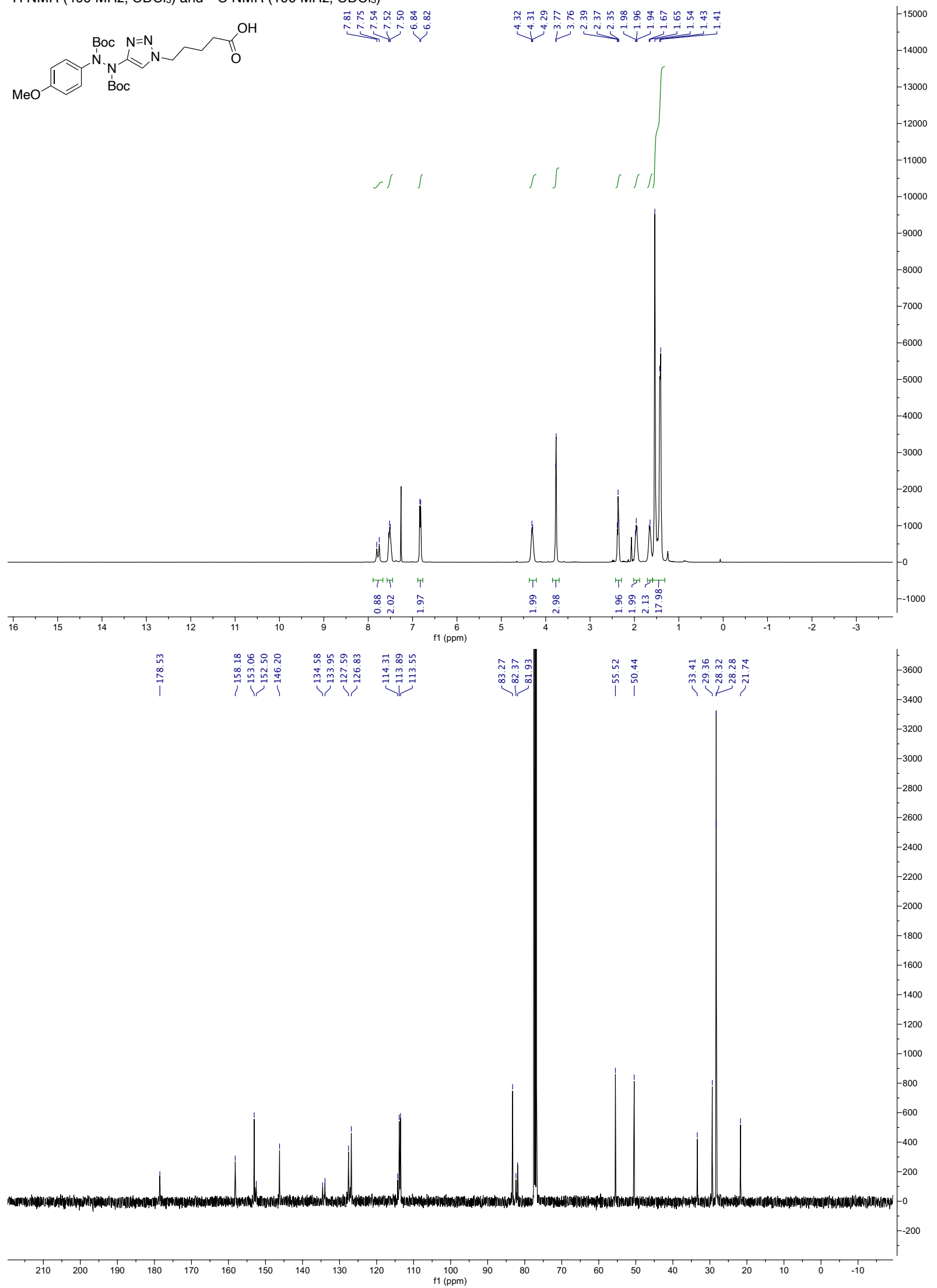


${ }^{1} \mathrm{H}$ NMR (400 MHz, DMSO- $\left.d_{6}\right)$ and ${ }^{13} \mathrm{C}$ NMR (100 MHz, DMSO- $\left.d_{6}\right)$

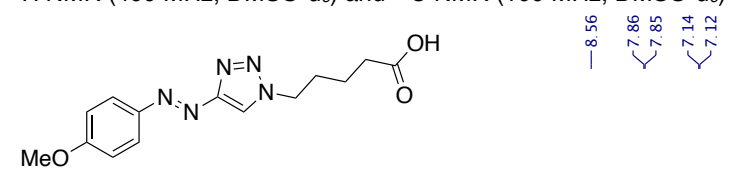

15

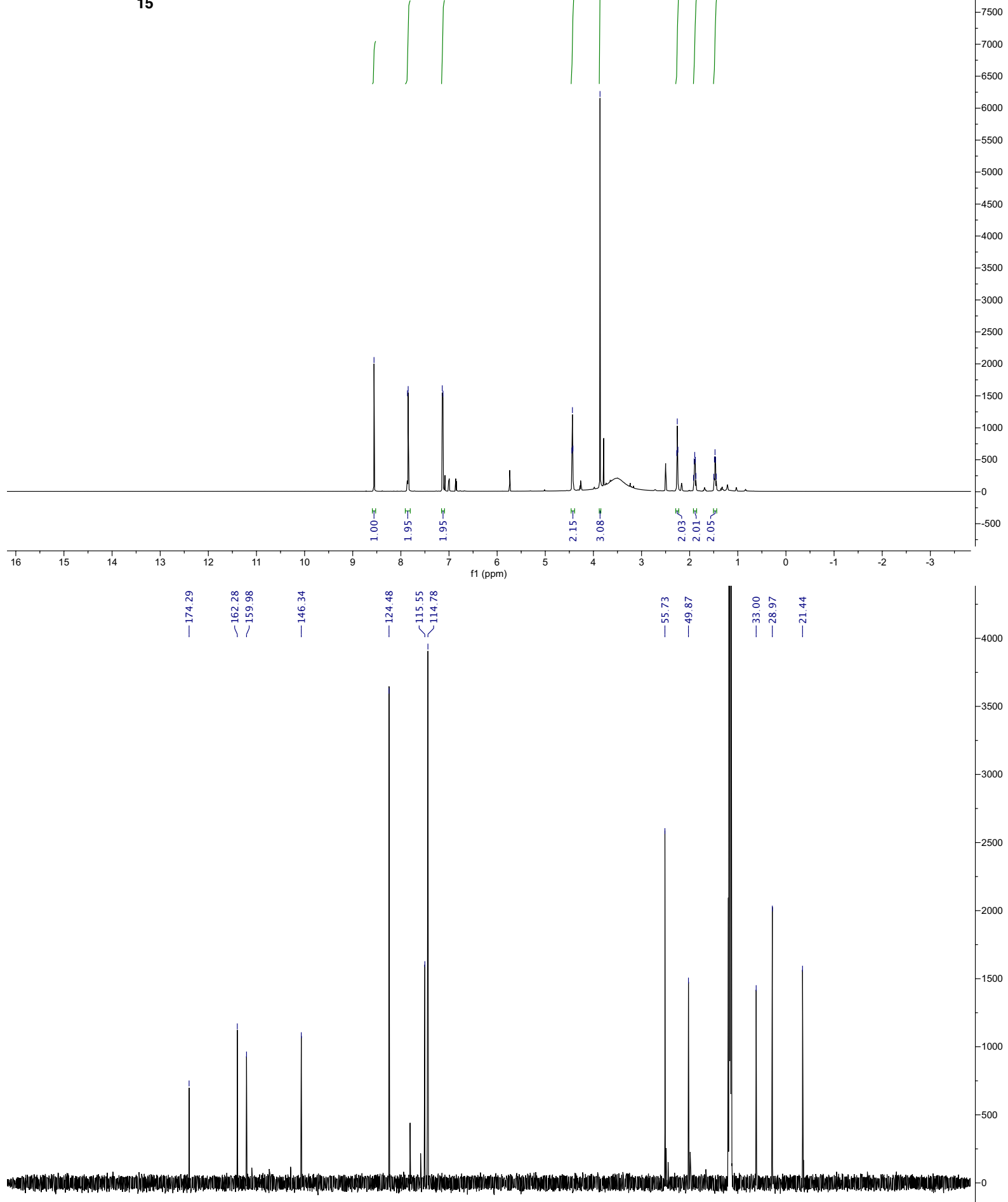


${ }^{1} \mathrm{H}$ NMR (400 MHz, $\left.\mathrm{CDCl}_{3}\right)$ and $\left.{ }^{13} \mathrm{C} \mathrm{NMR} \mathrm{(100} \mathrm{MHz,} \mathrm{CDCl}_{3}\right)$
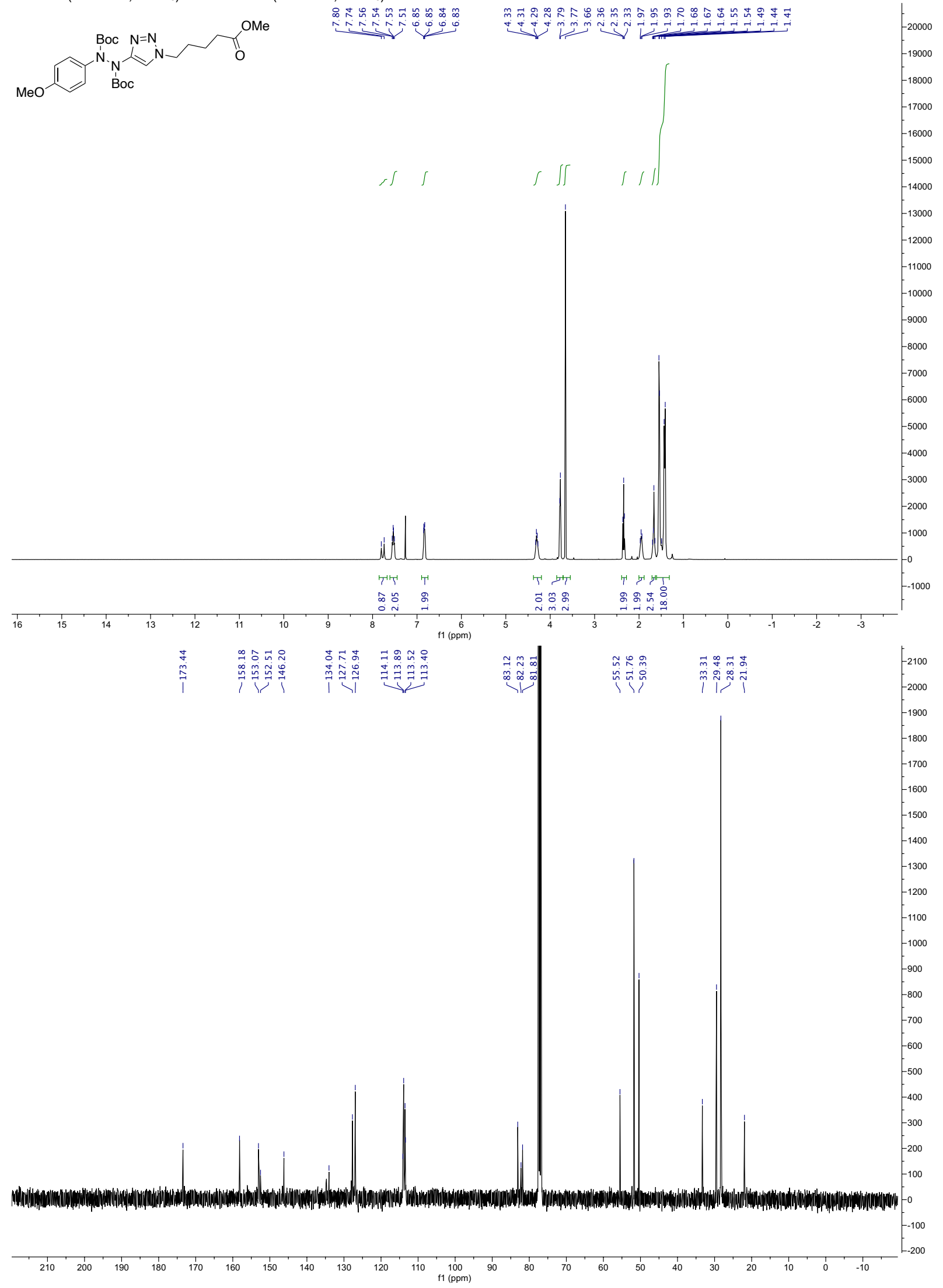
${ }^{1} \mathrm{H}$ NMR (400 MHz, $\left.\mathrm{CDCl}_{3}\right)$ and $\left.{ }^{13} \mathrm{C} \mathrm{NMR} \mathrm{(100} \mathrm{MHz,} \mathrm{CDCl}_{3}\right)$

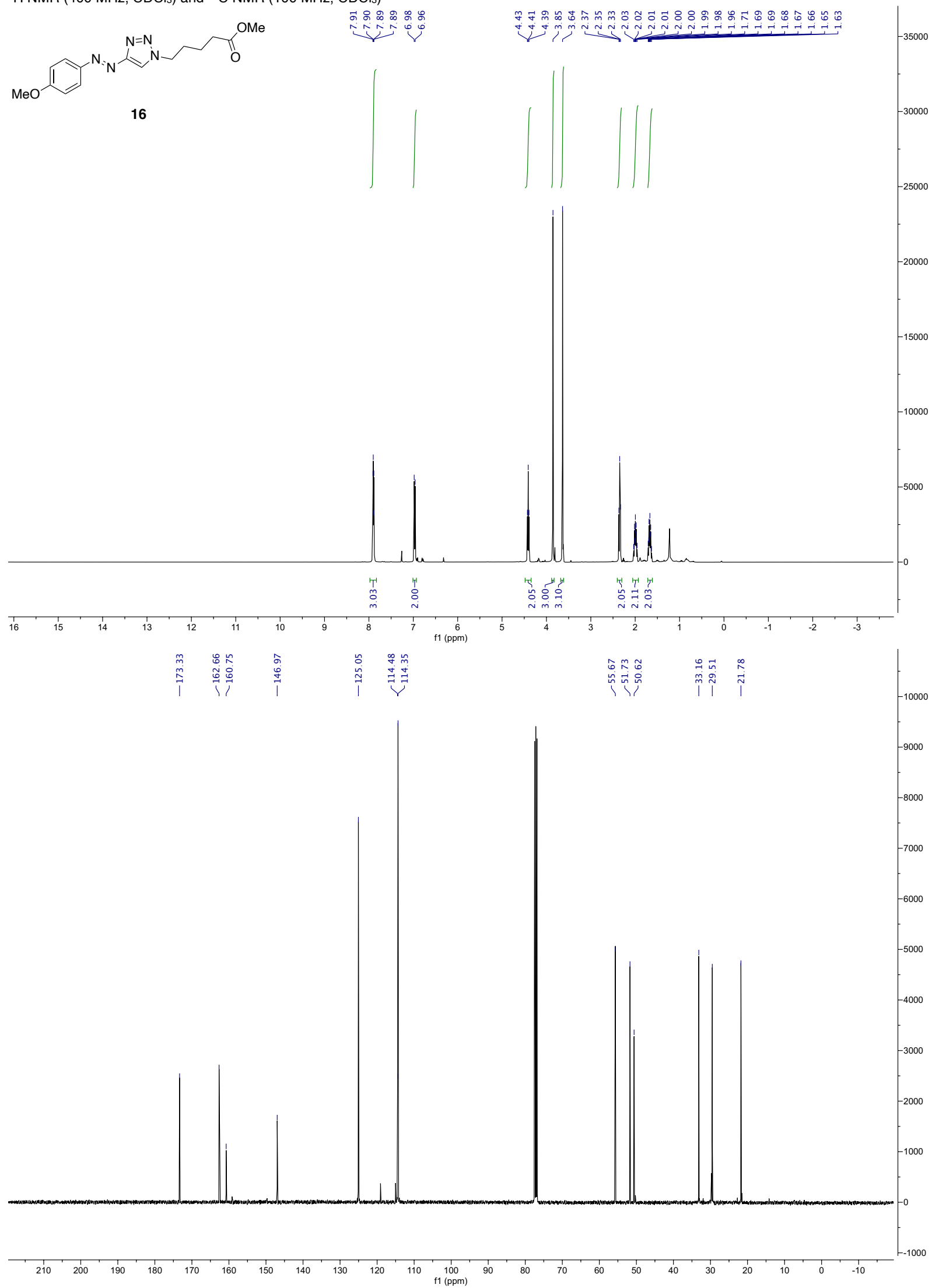


${ }^{1} \mathrm{H}$ NMR (400 MHz, $\left.\mathrm{CDCl}_{3}\right)$ and $\left.{ }^{13} \mathrm{C} \mathrm{NMR} \mathrm{(100} \mathrm{MHz,} \mathrm{CDCl}_{3}\right)$
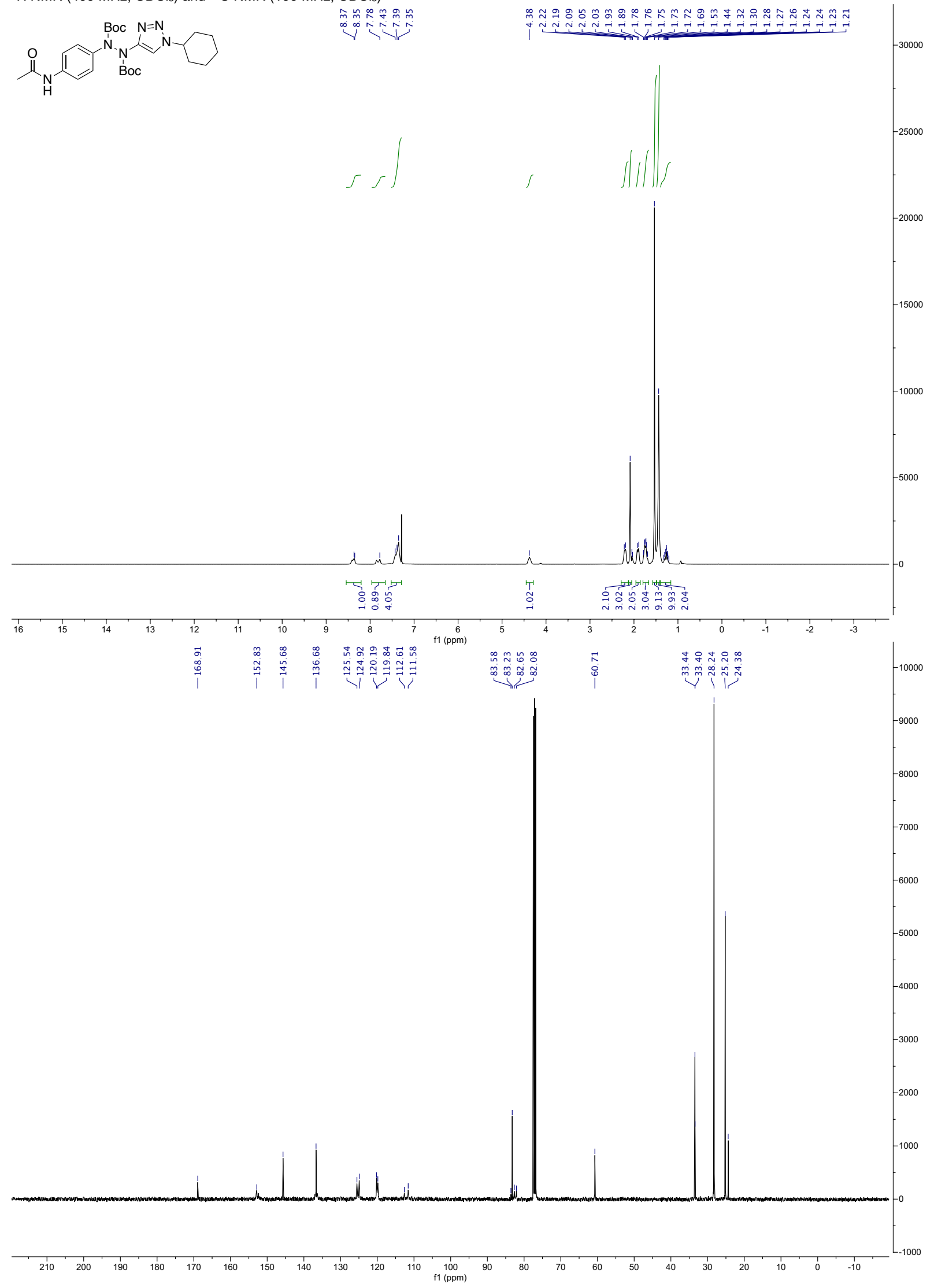


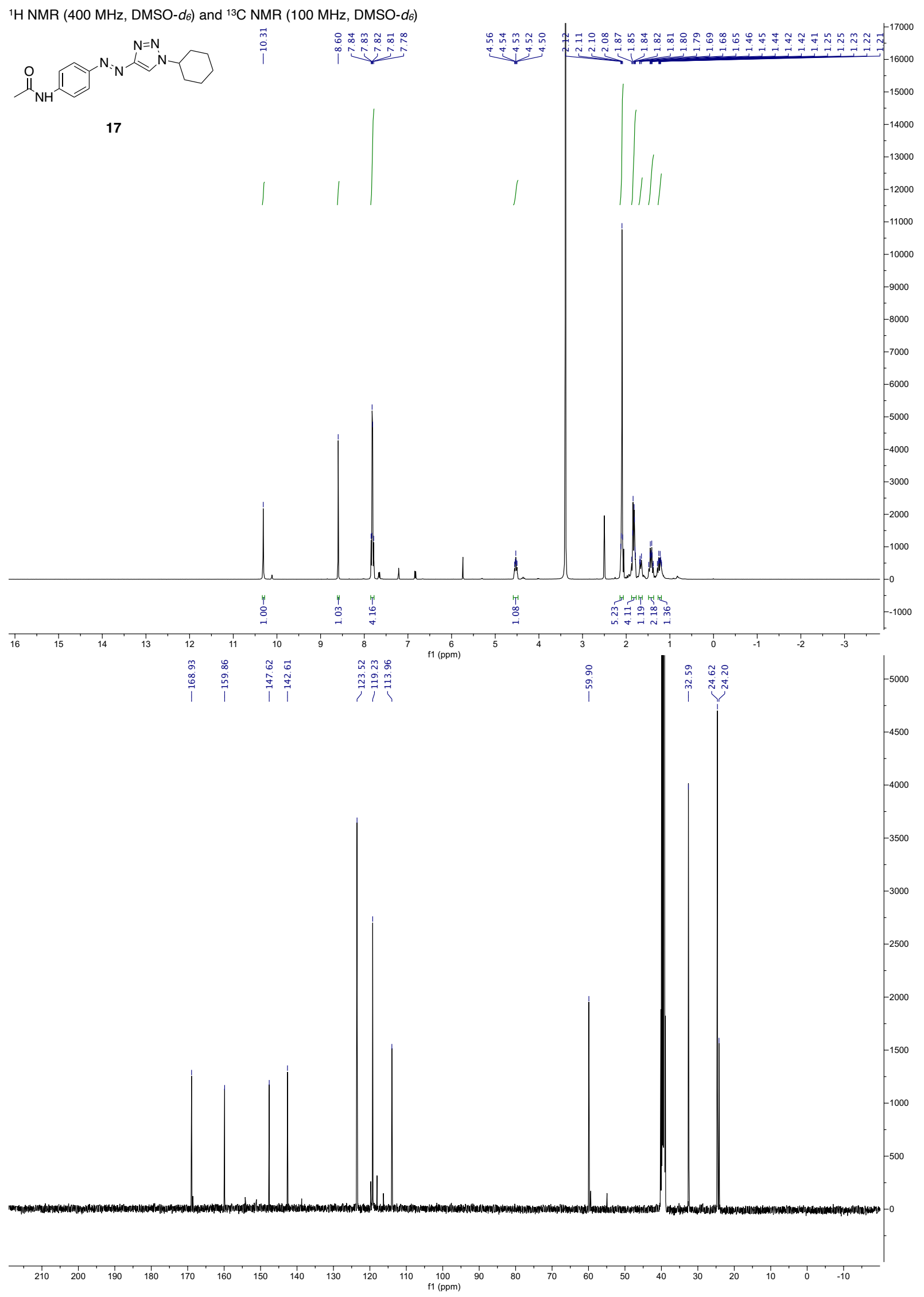


${ }^{1} \mathrm{H}$ NMR (400 MHz, $\mathrm{CDCl}_{3}$ ) and $\left.{ }^{13} \mathrm{C} \mathrm{NMR} \mathrm{(100} \mathrm{MHz,} \mathrm{CDCl}_{3}\right)$

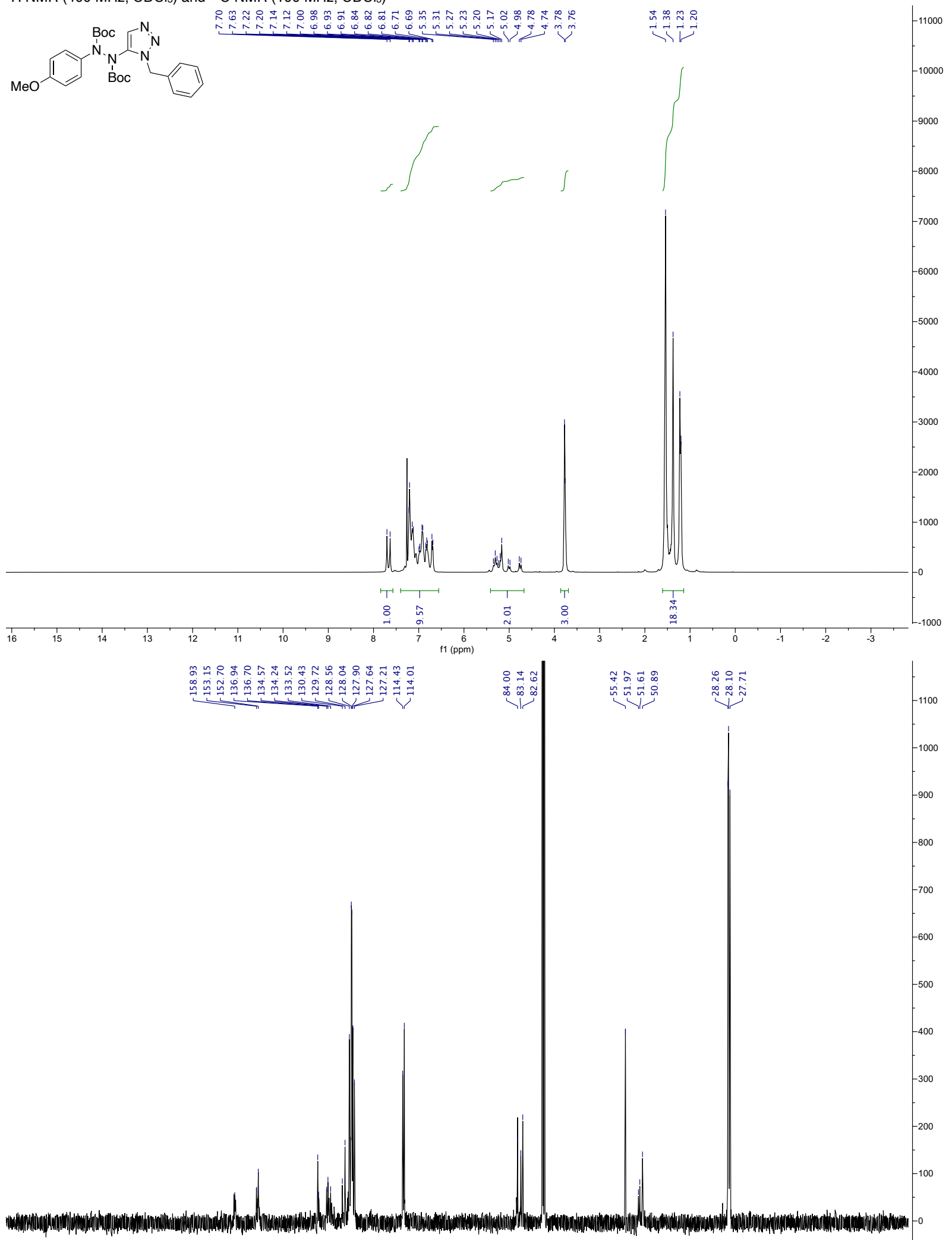

$-11000$ 000 
${ }^{1} \mathrm{H}$ NMR (400 MHz, $\mathrm{CDCl}_{3}$ ) and $\left.{ }^{13} \mathrm{C} \mathrm{NMR} \mathrm{(100} \mathrm{MHz,} \mathrm{CDCl}_{3}\right)$

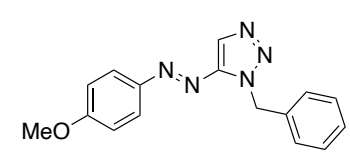

18

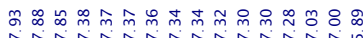

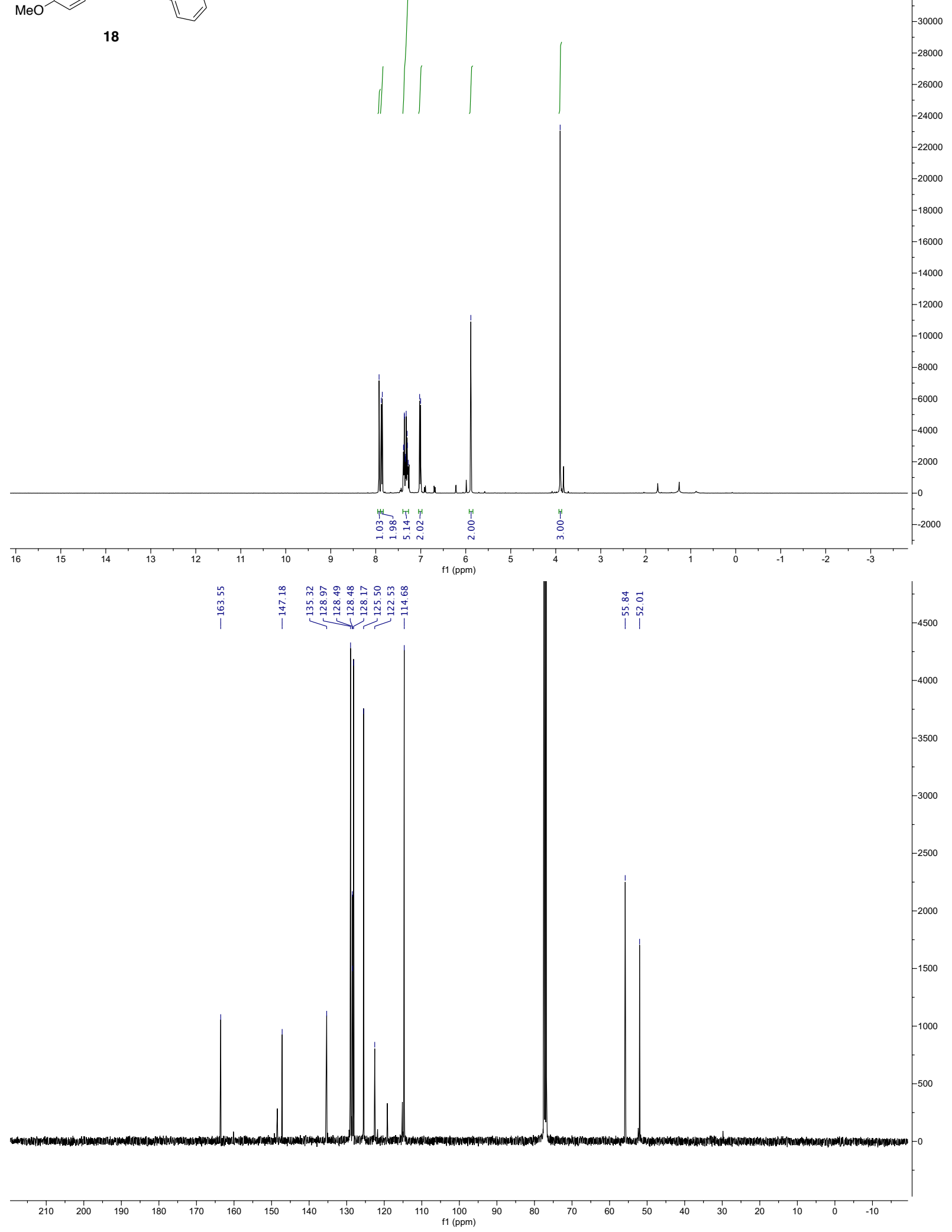


${ }^{1} \mathrm{H} \mathrm{NMR}\left(400 \mathrm{MHz}, \mathrm{CDCl}_{3}\right)$ and $\left.{ }^{13} \mathrm{C} \mathrm{NMR} \mathrm{(100} \mathrm{MHz,} \mathrm{CDCl}_{3}\right)$

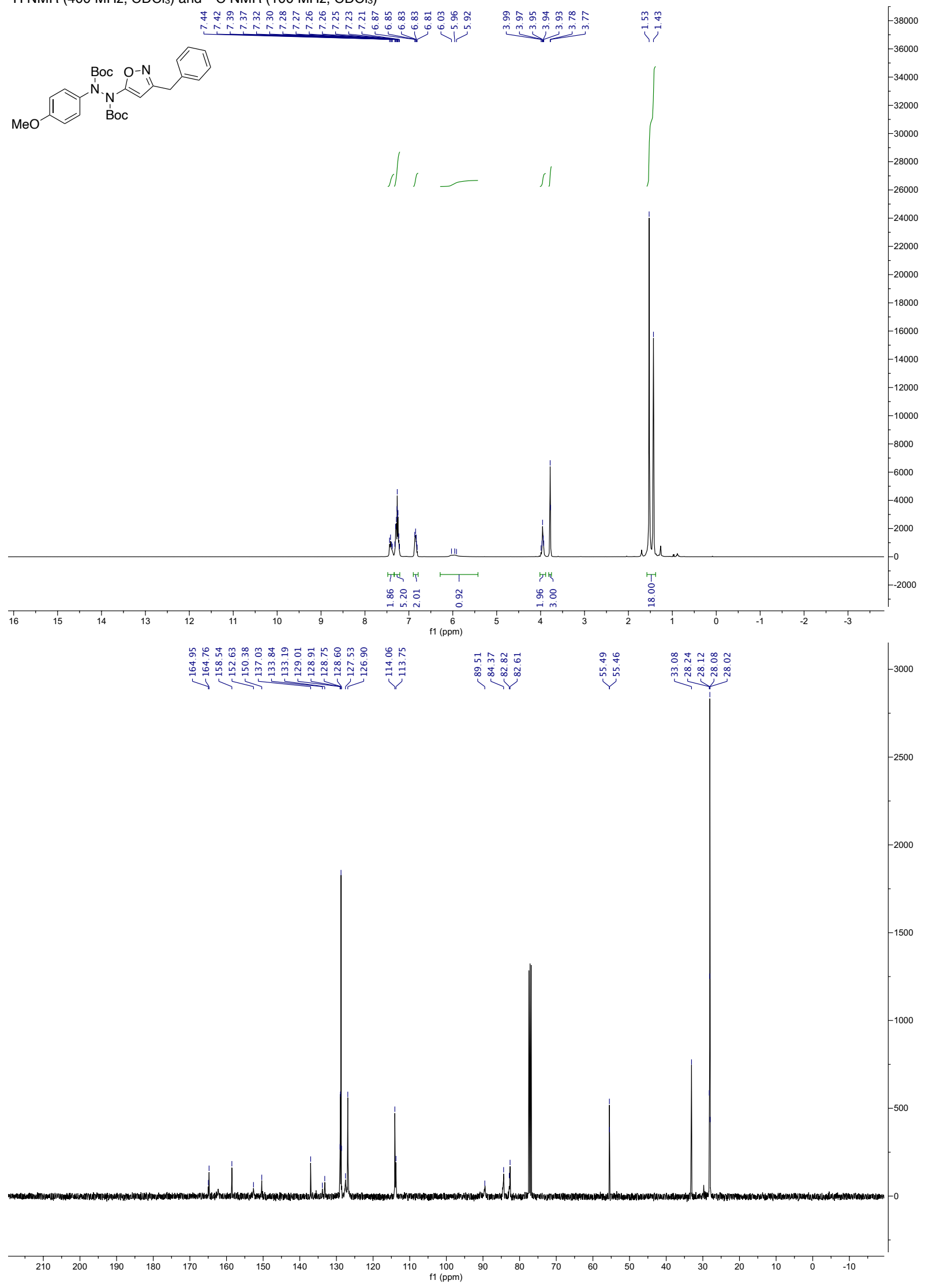


${ }^{1} \mathrm{H} \mathrm{NMR}\left(400 \mathrm{MHz}, \mathrm{CDCl}_{3}\right)$ and ${ }^{13} \mathrm{C} \mathrm{NMR}\left(100 \mathrm{MHz}, \mathrm{CDCl}_{3}\right)$

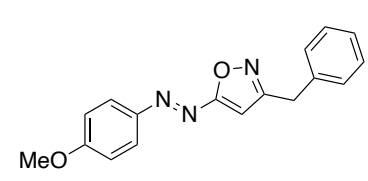

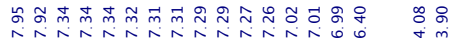

19

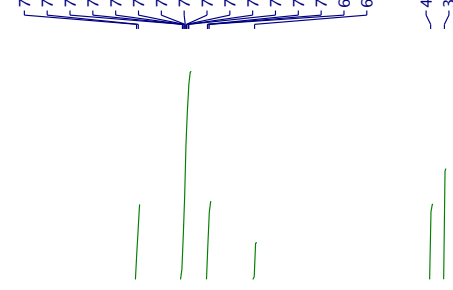

$-21000$
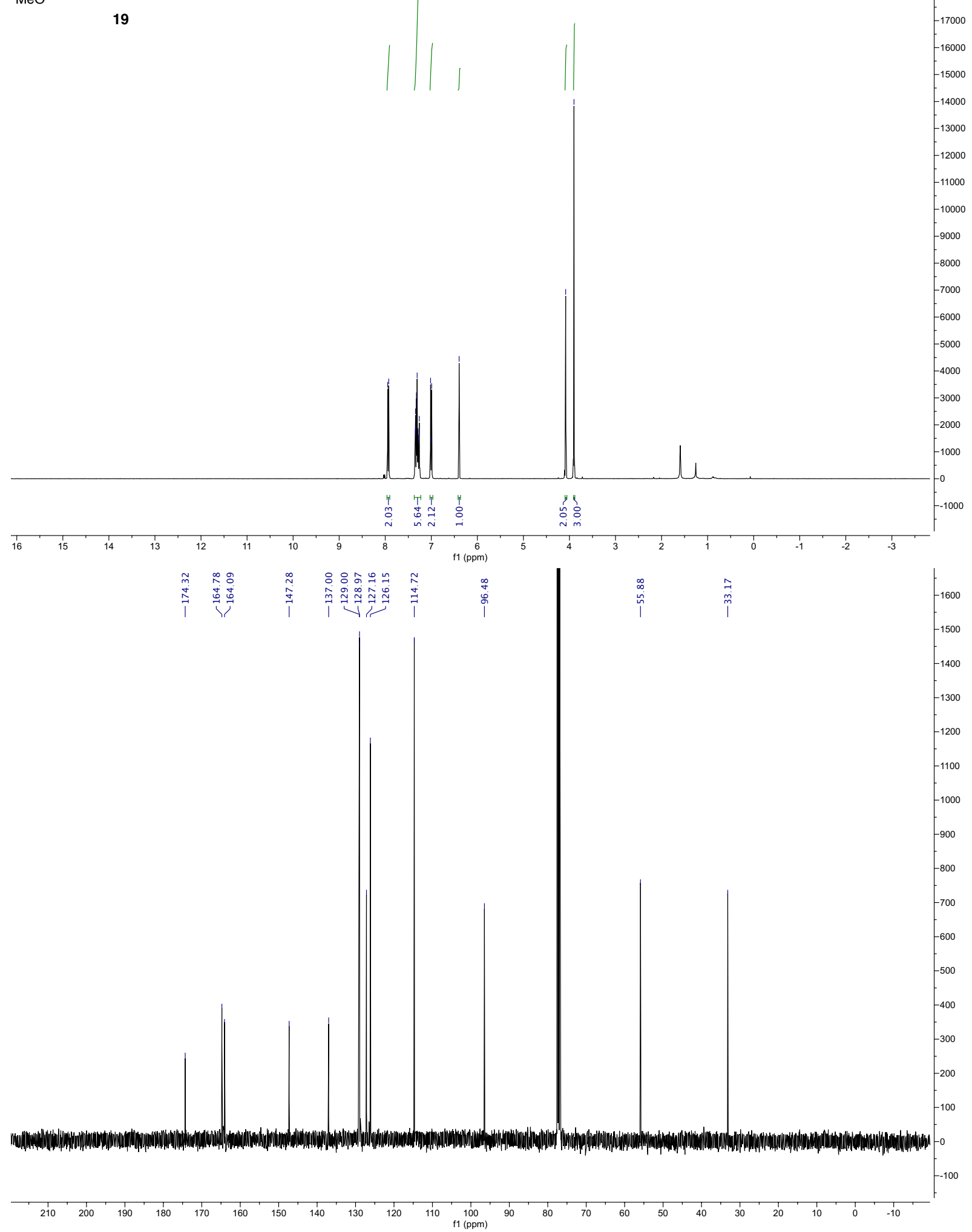